\title{
MODULI SPACES FOR DYNAMICAL SYSTEMS WITH PORTRAITS
}

\author{
JOHN R. DOYLE AND JOSEPH H. SILVERMAN
}

\begin{abstract}
A portrait $\mathcal{P}$ on $\mathbb{P}^{N}$ is a pair of finite point sets $Y \subseteq$ $X \subset \mathbb{P}^{N}$, a map $Y \rightarrow X$, and an assignment of weights to the points in $Y$. We construct a parameter space $\operatorname{End}_{d}^{N}[\mathcal{P}]$ whose points correspond to degree $d$ endomorphisms $f: \mathbb{P}^{N} \rightarrow \mathbb{P}^{N}$ such that $f: Y \rightarrow X$ is as specified by a portrait $\mathcal{P}$, and prove the existence of the GIT quotient moduli space $\mathcal{M}_{d}^{N}[\mathcal{P}]:=\operatorname{End}_{d}^{N} / / \mathrm{SL}_{N+1}$ under the $\mathrm{SL}_{N+1}$-action $(f, Y, X)^{\phi}=\left(\phi^{-1} \circ f \circ \phi, \phi^{-1}(Y), \phi^{-1}(X)\right)$ relative to an appropriately chosen line bundle. We also investigate the geometry of $\mathcal{M}_{d}^{N}[\mathcal{P}]$ and give two arithmetic applications.
\end{abstract}

\section{Contents}

1. Introduction

2. Earlier Results

3. The Category of Abstract Portraits

4. A Brief Summary of Geometric Invariant Theory 8

5. Notation and Basic Properties of $\operatorname{End}_{d}^{N}$

6. Models of Portraits and Portrait Moduli Problems 11

7. The Parameter Space $\operatorname{End}_{d}^{N}[\mathcal{P}]$ for Models of a Portrait 14

8. The $\mathrm{SL}_{N+1}$-Stable Locus of $\operatorname{End}_{d}^{N} \times\left(\mathbb{P}^{N}\right)^{n}$

9. Formal Models and Stability of $\operatorname{End}_{d}^{N}[\mathcal{P}]$

10. The Moduli Space $\mathcal{M}_{d}^{N}[\mathcal{P}]$ for Portrait Models 39

11. Refined Stability Criteria for $\mathbb{P}^{1}$

12. The Moduli Space of $\mathbb{P}^{1}$-Endomorphisms with Marked Critical Points

14. The Dimension of $\mathcal{M}_{d}^{N}[\mathcal{P}]$ for Unweighted Portraits 56

15. The Dimension of $\mathcal{M}_{d}^{1}[\mathcal{P}]$ for Weighted Portraits 63

16. An FOD/FOM Degree Bound for Dynamical Systems with Portraits

Date: December 27, 2018.

2010 Mathematics Subject Classification. Primary: 37P45; Secondary: 37P15.

Key words and phrases. portrait, dynamical system, arithmetic dynamics.

Silverman's research supported by Simons Collaboration Grant \#241309. 
17. Uniform Boundedness of Preperiodic Points and Its Relation to Algebraic Points on $\mathcal{M}_{d}^{N}[\mathcal{P}]$

18. Good Reduction of Dynamical Systems

19. Multiplier Systems for Periodic Portraits

\section{INTRODUCTION}

The primary goal of this paper is the construction of moduli spaces for dynamical systems that come equipped with a collection of points having specified (partial) orbits and weights. We start with the space $\operatorname{End}_{d}^{N}$ of degree $d$ endomorphisms $f: \mathbb{P}^{N} \rightarrow \mathbb{P}^{N}$ and the natural conjugation action of $\varphi \in \operatorname{Aut}\left(\mathbb{P}^{N}\right) \cong \mathrm{PGL}_{N+1}$ on $\operatorname{End}_{d}^{N}$ given by $f^{\varphi}:=\varphi^{-1} \circ f \circ \varphi$. The maps $f$ and $f^{\varphi}$ have the same dynamics, so the quotient space $\mathcal{M}_{d}^{N}:=\operatorname{End}_{d}^{N} / / \mathrm{PGL}_{N+1}$ is the moduli space of degree $d$ dynamical systems on $\mathbb{P}^{N}$. It is well-known that $\mathcal{M}_{d}^{N}$ exists as a GIT quotient space; see [38, 46, 51, 57].

We now add a portrait structure, which is a natural dynamical notion of level structure, to a rational map $f$. Roughly speaking, we aim to classify triples $(f, Y, X)$, where $Y \subset X \subset \mathbb{P}^{N}$ are finite sets of points such that $f(Y) \subset X$ and such that the map $f: Y \rightarrow X$ has a specified dynamical structure. Our goal is to construct a moduli space that classifies the set of such triples, modulo the action of $\varphi \in \mathrm{PGL}_{N+1}$ via $(f, Y, X)^{\varphi}:=\left(f^{\varphi}, \varphi^{-1}(X), \varphi^{-1}(Y)\right)$.

For example, we might take $Y=X$ and require $f: Y=X \rightarrow X$ to be the identity map, in which case we are classifying maps together with a collection of fixed points. As a second example with $Y=X$, we might require that $f: Y=X \rightarrow X$ be a cyclic permutation, so we are classifying maps together with a cycle of a given period. More generally, we may further assign weights to the points in $Y$ and require that the multiplicity of $f$ exceed the specified multiplicity. In this way, for example, we obtain a moduli space for maps on $\mathbb{P}^{1}$ whose critical points have a specified orbit structure, a topic that has been much studied due to its importance in complex dynamics. Leaving formal definitions for later, we briefly state some of our main results.

Theorem 1.1. Let $N \geq 1$ and $d \geq 2$, and let $\mathcal{P}$ be a portrait. Then with appropriate choices of structure sheaves, the following are true:

(a) There is a $\mathbb{Z}$-scheme $\mathcal{M}_{d}^{N}[\mathcal{P}]$ that classifies equivalence classes of dynamical systems on $\mathbb{P}^{N}$ with marked points that model $\mathcal{P}$; see Theorem 10.1. 
(b) If either $\mathcal{P}$ is unweighted or $N=1$, and if the variety $\mathcal{M}_{d}^{N}[\mathcal{P}] \otimes_{\mathbb{Z}}$ $\mathbb{Q}$ is not empty, then it has the expected dimension; see Theorems 14.2 and 15.7 .

We refer the reader to Section 3 for the formal definition of abstract portraits and to Section [6 for the description of the moduli problem for an endomorphism of $\mathbb{P}^{N}$ to model an weighted portrait.

It often happens that a portrait has non-trivial automorphisms. For example, a collection of fixed points may be arbitrarily permuted, while an $n$-cycle may be cyclically permuted. In the setting of Theorem 1.1 , for $\mathcal{A} \subseteq \operatorname{Aut}(\mathcal{P})$, we construct more general moduli spaces $\mathcal{M}_{d}^{N}[\mathcal{P} \mid \mathcal{A}]$ that classify dynamical systems with marked points that model $\mathcal{P}$, modulo the action of $\mathcal{A}$. For example, if $\mathcal{P}$ is a single $n$-cycle and $\mathcal{A} \cong$ $\mathbb{Z} / n \mathbb{Z}$ is the group of cyclic permutations, then $\mathcal{M}_{d}^{N}[\mathcal{P}]$ classifies maps $f$ with $n$ marked points forming an $n$-cycle, while $\mathcal{M}_{d}^{N}[\mathcal{P} \mid \mathcal{A}]$ classifies maps $f$ together with an $n$-cycle of points, where the points in the $n$-cycle are indistinguishable 1

It is a truth universally acknowledged that algebro-geometric objects in possession of an underlying structure must be in want of a moduli space.2 This provides our primary justification for constructing and studying $\mathcal{M}_{d}^{N}[\mathcal{P}]$, but we also briefly describe two arithmetic applications. The first concerns the dynamical uniform boundedness conjecture, whose exact statement is given in Section 17. We prove that this conjecture is equivalent to a statement about algebraic points on $\mathcal{M}_{d}^{N}[\mathcal{P}]$; see Theorem [17.5. To do this, we must first extend a fieldof-moduli versus field-of-definition result from an earlier paper [19]; see Section 16. The second application concerns good reduction of dynamical systems with portrait structure. In Section 18 we briefly discuss dynamical good reduction and its relationship to integral points on the moduli space $\mathcal{M}_{d}^{N}[\mathcal{P}$ ]; we refer the reader to [56] for a more extensive discussion of this last topic.

We close this introduction with a summary of the contents of our paper. Section 2 surveys the literature on dynamical moduli spaces. Section 3 describes the abstract theory of portraits, which are "pictures" that are used to classify "maps with orbits." Sections 4 and 5 briefly recall notation and properties, the former from geometric invariant theory, the latter for the space $\operatorname{End}_{d}^{N}$. In Section 6 we define models

\footnotetext{
${ }^{1}$ For those who are familiar with elliptic modular curves, we can make an analogy with the curves $X_{1}(n)$ and $X_{0}(n)$. The former classifies pairs $(E, P)$ consisting of an elliptic curve and a point of exact order $n$, while the latter classifies pairs $(E, C)$ consisting of an elliptic curve and a cyclic subgroup of order $n$. Then $X_{0}(n)$ is the quotient of $X_{1}(n)$ via the natural action of $(\mathbb{Z} / n \mathbb{Z})^{\times}$on $X_{1}(n)$.

2 ... with apologies to J.A.
} 
of portraits and the associated parameter and moduli problems. Section 7 is devoted to constructing parameter spaces $\operatorname{End}_{d}^{N}[\mathcal{P}]$ for maps with portraits, first for unweighted portraits, and then for weighted portraits. We next turn in Section 8 to GIT and show that $\operatorname{End}_{d}^{N} \times\left(\mathbb{P}^{N}\right)^{n}$ is $\mathrm{SL}_{N+1}$-stable relative to a suitably chosen line bundle. We use this result and a covering lemma in Section 9 to construct the moduli space space $\mathcal{M}_{d}^{N}[\mathcal{P}]$ classifying $\mathrm{SL}_{N+1}$-isomorphism classes of maps with portraits, and more generally we construct moduli spaces $\mathcal{M}_{d}^{N}[\mathcal{P} \mid \mathcal{A}]$ obtained by taking the quotient by a group $\mathcal{A}$ of automorphisms of $\mathcal{P}$. Section 12 contains a more precise description of $\mathcal{M}_{d}^{1}[\mathcal{P}]$ in the case that $\mathcal{P}$ is a complete preperiodic marking of the critical points. We then use Section 13 to describe a number of explicit examples of $\mathcal{M}_{d}^{1}[\mathcal{P}]$, and more generally $\mathcal{M}_{d}^{1}[\mathcal{P} \mid \mathcal{A}]$, in both the unweighted and weighted cases. Returning to the general theory, we note that there may be geometric or topological reasons that force $\mathcal{M}_{d}^{N}[\mathcal{P}]=\emptyset$. Assuming that $\mathcal{M}_{d}^{N}[\mathcal{P}]$ is non-empty, we compute its dimension in Section 14 for all $N$ and unweighted portraits, and in Section 15 for $N=1$ and weighted portraits. Sections 16, 17, and 18 give arithmetic applications, as discussed earlier. We conclude in Section [19] with a discussion of multiplier systems. These are used to construct regular maps from $\mathcal{M}_{d}^{N}[\mathcal{P}]$ to affine space whose images are constrained by Ueda's fixed point multiplier relations.

\section{EARLIER Results}

In this section we briefly summarize some of the work that has been done on moduli spaces for dynamical systems and relate it to the present work. Our discussion provides a path into the existing literature, but is far from exhaustive.

Various general constructions of parameter and moduli spaces for dynamical systems on projective space have been given by a variety of people, including:

- Degree 2 maps on $\mathbb{P}^{1}$ : DeMarco [10, Milnor [46], Rees [54, 55].

- Degree 3 maps on $\mathbb{P}^{1}$ : Hutz-Tepper [31, West [61, 62.

- Degree $d$ maps on $\mathbb{P}^{1}$ : Silverman [57].

- Degree $d$ maps on $\mathbb{P}^{N}$ : Levy [38], Petsche-Szpiro-Tepper [51].

Turning now to dynamical systems with marked points, there is a large literature studying so-called dynatomic curves, which are 1dimensional moduli spaces whose points classify pairs consisting of a map in a 1-parameter family and a point of given period or preperiod. The classical example is the dynatomic curve $X_{1}^{\text {dyn }}(n)$ whose 
points classify pairs $(c, \alpha)$ such that $\alpha$ is a point of exact (or formal) period $n$ for the map $x^{2}+c$, or more generally $x^{d}+c$. (See Example 13.1 for further information about dynatomic curves and their description as portrait moduli spaces.) The following papers are among those that investigate $X_{1}^{\text {dyn }}(n)$ : Bousch [5], Buff-EpsteinKoch [6], Buff-Lei [7], Douady-Hubbard [13, 14, Doyle [16], Doyle et al. [17], Doyle-Poonen [18], Gao [24], Gao-Ou [25], Krumm [34, 35], Lau-Schleicher [36], Morton [48]. These papers study topics such as smoothness, irreducibility, genus, and gonality of $X_{1}^{\mathrm{dyn}}(n)$, as well as reduction $\bmod p$ and specialization properties.

There are also many papers that study spaces of maps on $\mathbb{A}^{1}$ or $\mathbb{P}^{1}$ with the property that some or all of their critical points match a specified preperiodic portrait. A fundamental result in this area is Thurston's rigidity theorem, see [15, 37], which we will make crucial use of in our investigations. Among other research in this area, we mention work of Bielefeld-Fisher-Hubbard [2] and Poirier [52] that describes all critical point portraits that can arise from polynomial maps on $\mathbb{A}^{1}$, a paper of Cordwell et al. 9] classifying rational functions on $\mathbb{P}^{1}$ whose critical points are all fixed points, and papers of Faber-Granville [20], Ghioca-Nguyen-Tucker [26], and Ingram-Silverman [32] that describe preperiodic portraits that occur under reduction modulo primes.

The literature on higher dimensional moduli spaces classifying dynamical systems with marked points is smaller. Probably closest in spirit to the present paper is recent work of Arfeux [1, in which he constructs a moduli space for triples $(f, Y, Z)$, where $f: \mathbb{P}^{1} \rightarrow \mathbb{P}^{1}$ is a rational map of degree $d$, and where $Y, Z \subset \mathbb{P}^{1}$ specify marked sets of points such that $Y=f^{-1}(Z)$ and such that $Z$ contains the critical values of $f$. Arfeux defines an abstract portrait to be the 4-tuple $(Y, Z, Y \rightarrow Z$, deg $: Y \rightarrow \mathbb{N})$, so more-or-less the same as our portrait data $\left(\mathcal{V}^{\circ}, \mathcal{V}, \Phi, \epsilon\right)$. Our construction, in addition to being somewhat more general for $\mathbb{P}^{1}$, also deals with portrait moduli spaces for dynamical systems on $\mathbb{P}^{N}$. We note that our work is primarily algebraic in nature, while Arfeux's work has a more geometric flavor. The two papers thus provide complementary approaches, both of which are useful.

\section{The Category of Abstract Portraits}

In this section we describe an abstract theory of portraits, which are the "pictures" that we use to classify "maps with orbits."

Definition 3.1. A portrait is a 4-tuple

$$
\mathcal{P}=\left(\mathcal{V}^{o}, \mathcal{V}, \Phi, \epsilon\right)
$$


such that:

- $\mathcal{V}$ is a finite set.

- $\mathcal{V}^{\circ} \subseteq \mathcal{V}$ is a subset.

- $\Phi: \mathcal{V}^{\circ} \rightarrow \mathcal{V}$ is a function.

- $\epsilon: \mathcal{V}^{\circ} \rightarrow \mathbb{Z}_{\geq 1}$ is a (multiplicity or weight) function.

A portrait is unweighted if $\epsilon(w)=1$ for every $w \in \mathcal{V}^{\circ}$, in which case we write $\mathcal{P}=\left(\mathcal{V}^{\circ}, \mathcal{V}, \Phi\right)$. We say that $\mathcal{P}$ is preperiodic if $\mathcal{V}=\mathcal{V}^{\circ}$, or equivalently, since $\mathcal{V}$ is finite, if every element of $\mathcal{V}^{\circ}$ has a well-defined closed forward orbit under the map $\Phi$. In this case we may omit $\mathcal{V}^{\circ}$ from the notation and write simply $(\mathcal{V}, \Phi, \epsilon)$, or $(\mathcal{V}, \Phi)$.

Definition 3.2. Let $\mathcal{P}=\left(\mathcal{V}^{o}, \mathcal{V}, \Phi, \epsilon\right)$ be a portrait. The set of critical points of $\mathcal{P}$ is

$$
\operatorname{Crit}(\mathcal{P}):=\left\{v \in \mathcal{V}^{\circ}: \epsilon(v) \geq 2\right\}
$$

The (forward) orbit of a point $v \in \mathcal{V}^{\circ}$ is the set

$$
\mathcal{O}_{\Phi}(v):=\left\{\Phi^{n}(v): n \geq 0 \text { and } \Phi^{n-1}(v) \in \mathcal{V}^{\circ}\right\}
$$

where we abuse notation and let $\Phi^{0}(v)=v$ for any $v \in \mathcal{V}$. In other words, $\mathcal{O}_{\Phi}(v)$ is the set of points obtained by starting at $v$ and repeatedly applying $\Phi$ until reaching a point where $\Phi$ is not defined.

Definition 3.3. Let $\mathcal{P}=\left(\mathcal{V}^{\circ}, \mathcal{V}, \Phi_{\mathcal{V}}, \epsilon_{\mathcal{V}}\right)$. A subportrait of $\mathcal{P}$ is a portrait $\left(\mathcal{W}^{\circ}, \mathcal{W}, \Phi_{\mathcal{W}}, \epsilon_{\mathcal{W}}\right)$ satisfying

$$
\mathcal{W} \subseteq \mathcal{V}, \quad \mathcal{W}^{\circ} \subseteq \mathcal{V}^{\circ}, \quad \Phi_{\mathcal{W}}=\left.\Phi_{\mathcal{V}}\right|_{\mathcal{W}^{\circ}}, \quad \epsilon_{\mathcal{W}} \leq\left.\epsilon_{\mathcal{V}}\right|_{\mathcal{W}^{\circ}}
$$

Definition 3.4. Let $\mathcal{P}_{1}=\left(\mathcal{V}_{1}^{\circ}, \mathcal{V}_{1}, \Phi_{1}, \epsilon_{1}\right)$ and $\mathcal{P}_{2}=\left(\mathcal{V}_{2}^{\circ}, \mathcal{V}_{2}, \Phi_{2}, \epsilon_{2}\right)$ be portraits. A portrait morphism from $\mathcal{P}_{1}$ to $\mathcal{P}_{2}$ is an injective set map

$$
\alpha: \mathcal{V}_{1} \longleftrightarrow \mathcal{V}_{2}
$$

satisfying:

- $\alpha\left(\mathcal{V}_{1}^{\circ}\right) \subseteq \mathcal{V}_{2}^{\circ}$.

- $\alpha \circ \Phi_{1}(v)=\Phi_{2} \circ \alpha(v)$ for all $v \in \mathcal{V}_{1}^{\circ}$.

- $\epsilon_{2} \circ \alpha(v) \geq \epsilon_{1}(v)$ for all $v \in \mathcal{V}_{1}^{\circ}$.

We say $\alpha$ is a portrait isomorphism if it has a two-sided inverse, or equivalently, if it satisfies

$$
\alpha\left(\mathcal{V}_{1}\right)=\mathcal{V}_{2}, \quad \alpha\left(\mathcal{V}_{1}^{\circ}\right)=\mathcal{V}_{2}^{\circ}, \quad \epsilon_{2} \circ \alpha(v)=\epsilon_{1}(v) \text { for all } v \in \mathcal{V}_{1}^{\circ}
$$

We write $\operatorname{Hom}\left(\mathcal{P}_{1}, \mathcal{P}_{2}\right)$ for the set of portrait morphisms from $\mathcal{P}_{1}$ to $\mathcal{P}_{2}$, and $\operatorname{Aut}(\mathcal{P})$ for the group of portrait isomorphisms from a portrait $\mathcal{P}$ to itself. We note that $\operatorname{Aut}(\mathcal{P})$ is a subgroup of the symmetric group on the set $\mathcal{V}$ of vertices of $\mathcal{P}$.

Note that we have defined a category $\mathfrak{P o r t}$ whose objects are portraits and whose morphisms are portrait morphisms. Subcategories 
include the category of unweighted portraits and the category of preperiodic portraits.

Table 1 lists the automorphism groups of some unweighted preperiodic portraits whose vertex set contains 4 points.

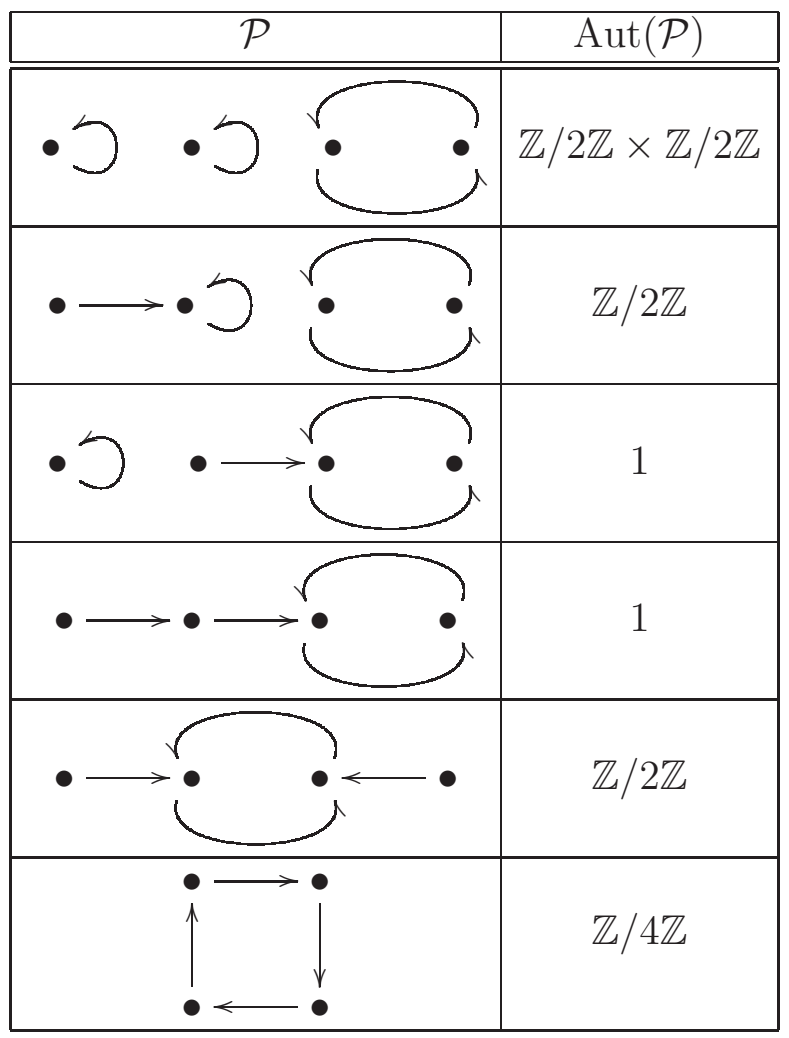

TABLE 1. Examples of Portrait Automorphism Groups

3.1. Portraits, Graphs, and Pseudoforests. We have called our objects portraits because that is what they are typically called in dynamical systems, especially in the study of critical point portraits. But our portraits can also be described using terminology from graph theory. Specifically, a portrait is a finite multi-directed pseudoforest. To unpack this phrase, we start with a multi-digraph, which is a directed graph $(V, E)$ that comes with a function $\mu: E \rightarrow \mathbb{Z}_{\geq 1}$ that assigns a multiplicity to each edge. We next require that it be a finite graph, which simply means that the set of vertices $V$ is finite. Finally, a directed graph is a pseudoforest if every vertex has at most one out-arrow, which is equivalent to the condition that every connected component 
of the graph is either a directed tree or a directed graph containing at most one cycle.

With these definitions, a portrait $\mathcal{P}=\left(\mathcal{V}^{\circ}, \mathcal{V}, \Phi, \epsilon\right)$ can be used to create a finite multi-directed pseudoforest $(V, E, \mu)$ by taking

$$
V=\mathcal{V} \quad \text { and } \quad E=\left\{(v, \Phi(v)): v \in \mathcal{V}^{\circ}\right\}
$$

and using the multiplicity function

$$
\mu(v, \Phi(v))=\epsilon(v) \quad \text { for } v \in \mathcal{V}^{\circ} .
$$

Ignoring the multiplicities, the directed graph is essentially the function graph of $\Phi$ in $\mathcal{V}^{\circ} \times \mathcal{V}$. Conversely, a finite multi-directed pseudoforest $(V, E, \mu)$ can be used to define a portrait by taking

$$
\begin{aligned}
\mathcal{V} & =V, \quad \mathcal{V}^{\circ}=\{v \in V: v \text { has an out-arrow }\}, \\
\Phi(v) & =\text { vertex at other end of } v \text { 's out-arrow, } \\
\epsilon(v) & =\mu(\text { arrow originating at } v) .
\end{aligned}
$$

This gives a bijection between the set of portraits and the set of finite multi-directed pseudoforests, but the portrait characterization seems more conducive to dynamical discussions.

\section{A Brief Summary of Geometric Invariant Theory}

We set notation and briefly recall some basic facts from geometric invariant theory. For details, see [11, 50, 59].

Let $X$ be a scheme, let $G$ be a reductive group that acts on $X$, let $\mathcal{L}$ be an invertible sheaf on $X$ with a $G$-linearization, and let $x \in X$ be a geometric point. We consider the following properties:

There is an integer $n \geq 0$ and a $G$-invariant global section $s \in$ $\Gamma\left(X, \mathcal{L}^{\otimes n}\right)$ such that:

(i) $s(x) \neq 0$.

(ii) $X_{s}:=\{s \neq 0\}$ is affine.

(iii) The action of $G$ on $X_{s}$ is closed, i.e., for every $x^{\prime} \in X_{s}$, the orbit $G x^{\prime}$ is a closed subset of $X_{s}$.

(iv) The stabilizer $\operatorname{Stab}_{G}(x):=\{\sigma \in G: \sigma x=x\}$ is 0-dimensional.

Definition 4.1. The set of semi-stable, respectively stable, points (relative to the sheaf $\mathcal{L}$ ) is the set

$$
\begin{array}{rll}
X^{\mathrm{ss}}(\mathcal{L}) & :=\{x \in X:(\mathrm{i}) \text { and (ii) are true }\} & \text { (semi-stable) } \\
X^{\text {stab }}(\mathcal{L}) & :=\{x \in X:(\mathrm{i}),(\mathrm{ii}),(\mathrm{iii}) \text { and (iv) are true }\} & \text { (stable) }
\end{array}
$$

A point that is not semi-stable is called unstable. 
Remark 4.2. There is some variation in the literature regarding the definition of stability. Thus Mumford [50, Section 1.4] defines $x \in X$ to be stable if conditions (i), (ii), and (iii) hold, while $x$ is properly stable if condition (iv) is also true. However, it seems to have become more common to include condition (iv) in the definition of stability; see for example the standard reference [11, page 115]. We further note that the Hilbert-Mumford numerical criterion for stability [50, Theorem 2.1] gives a criterion for stable points that includes condition (iv). This is not an entirely empty distinction, since as shown in [50], a geometric quotient exists even if (iv) is violated. For example, consider the diagonal action of $\mathrm{SL}_{2}$ on $\left(\mathbb{P}^{1}\right)^{2}$. Then (i)-(iii) hold on $\left(\mathbb{P}^{1}\right)^{2} \backslash \Delta$, while no points satisfy (iv). The geometric quotient $\left(\left(\mathbb{P}^{1}\right)^{2} \backslash \Delta\right) / / \mathrm{SL}_{2}$ exists and consists of a single point.

We now describe the Hilbert-Mumford numerical criterion; see [50, Theorem 2.1] for details. Let $\ell: \mathbb{G}_{m} \rightarrow \mathrm{SL}_{m+1}$ be a 1-parameter subgroup, and diagonalize the action

$$
\ell(\alpha)=\left(\begin{array}{cccc}
\alpha^{r_{0}} & & & \\
& \alpha^{r_{1}} & & \\
& & \ddots & \\
& & & \alpha^{r_{m}}
\end{array}\right), \quad \text { i.e., } \quad \ell(\alpha) \star \hat{x}_{i}=\alpha^{r_{i}} \hat{x}_{i}
$$

where $\left(\hat{x}_{0}, \ldots, \hat{x}_{m}\right)$ are coordinates on $\mathbb{A}^{m+1}$. The numerical invariant of $\ell$ at $x$ is

$$
\mu^{\mathcal{L}}(x, \ell):=\max \left\{-r_{i}: i \text { such that } \hat{x}_{i} \neq 0\right\} .
$$

Theorem 4.3 (Mumford [50]). Let $G \subset \mathrm{SL}_{m+1}$ act on $\mathbb{P}^{m}$, and let $P \in \mathbb{P}^{m}$ be a geometric point. Then

$$
\begin{aligned}
P \in\left(\mathbb{P}^{m}\right)^{\mathrm{ss}}\left(\mathcal{O}_{\mathbb{P}^{m}}(1)\right) & \Longleftrightarrow \quad \mu^{\mathcal{O}_{\mathbb{P}}(1)}(P, \ell) \geq 0 \text { for all } \ell: \mathbb{G}_{m} \hookrightarrow G . \\
P \in\left(\mathbb{P}^{m}\right)^{\mathrm{stab}}\left(\mathcal{O}_{\mathbb{P}^{m}}(1)\right) & \Longleftrightarrow \mu^{\mathcal{O}_{\mathbb{P}}(1)}(P, \ell)>0 \text { for all } \ell: \mathbb{G}_{m} \hookrightarrow G .
\end{aligned}
$$

\section{Notation And Basic Properties of $\operatorname{End}_{d}^{N}$}

In this section we set some standard notation that will be used throughout this paper, and we recall some of the basic properties of the space $\operatorname{End}_{d}^{N}$ that parameterizes degree $d$ endomorphisms of $\mathbb{P}^{N}$. For $N \geq 1$ and $d \geq 1$, we recall that there are $\left(\begin{array}{c}N+d \\ d\end{array}\right)$ monomials of degree $d$ in the variables $x_{0}, \ldots, x_{N}$. We let

$$
M=M(N, d):=(N+1)\left(\begin{array}{c}
N+d \\
d
\end{array}\right)-1,
$$


so $M+1$ is the total number of coefficients that appear in $N+1$ generic homogeneous polynomials of degree $d$.

We let

$$
\operatorname{End}_{d}^{N}:=\text { the space of degree } d \text { endomorphisms } \mathbb{P}^{N} \rightarrow \mathbb{P}^{N} .
$$

We denote elements

$$
f=\left[f_{0}, \ldots, f_{N}\right] \in \operatorname{End}_{d}^{N}
$$

using homogeneous polynomials $f_{i}$ of degree $d$ in the variables $x_{0}, \ldots, x_{N}$. For each $0 \leq \rho \leq N$, we denote the coefficients of each $f_{\rho}$ by

$$
f_{\rho}(\boldsymbol{x})=\sum_{|\boldsymbol{e}|=d} a_{\rho}(\boldsymbol{e}) \boldsymbol{x}^{\boldsymbol{e}}:=\sum_{\begin{array}{c}
e_{0}, \ldots, e_{N} \geq 0 \\
e_{0}+e_{1}+\cdots+e_{N}=d
\end{array}} a_{\rho}\left(e_{0}, \ldots, e_{n}\right) x_{0}^{e_{0}} \cdots x_{N}^{e_{N}},
$$

where the notation is self-explanatory. The coefficients of the $f_{\rho}$ are only defined up to multiplication by a non-zero scalar, so fixing some ordering for the coefficients, each $f$ corresponds to a point in $\mathbb{P}^{M}$, i.e., there is a natural embedding

$$
\operatorname{End}_{d}^{N} \longleftrightarrow \mathbb{P}^{M}
$$

and the $a_{\rho}(\boldsymbol{e})$ are global sections of $\mathcal{O}_{\mathbb{P}^{M}}(1)$. The image of this embedding is the complement of the vanishing locus of the Macaulay resultant $\mathcal{R}$, which is a homogeneous polynomial in the $a_{\rho}(\boldsymbol{e})$ of degree $(N+1) d^{N}$.

We define an action of $\mathrm{SL}_{N+1}$ on $\operatorname{End}_{d}^{N}$ and on $\mathbb{P}^{N}$ as follows: For $\varphi \in \mathrm{SL}_{N+1}, f \in \operatorname{End}_{d}^{N}$, and $P \in \mathbb{P}^{N}$, we set

$$
\varphi \star f:=\varphi^{-1} \circ f \circ \varphi \text { and } \varphi \star P:=\varphi^{-1}(P) .
$$

We note that the actions are designed to be compatible with composition and evaluation,

$$
\varphi \star\left(f^{k}\right)=(\varphi \star f)^{k} \text { and }(\varphi \star f)(\varphi \star P)=\varphi \star(f(P)) .
$$

For $f \in \operatorname{End}_{d}^{N}$, we write

$$
\operatorname{Aut}(f):=\left\{\varphi \in \mathrm{SL}_{N+1}: \varphi \star f=f\right\}
$$

for the stabilizer of $f$.

The action of $\mathrm{SL}_{N+1}$ on $\operatorname{End}_{d}^{N}$ extends to an action on $\mathbb{P}^{M}$, and the following classical result says that $\left(\mathbb{P}^{M}\right)^{\text {stab }}\left(\mathcal{O}_{\mathbb{P}^{M}}(1)\right)$ contains $\operatorname{End}_{d}^{N}$, i.e., the action of $\mathrm{SL}_{N+1}$ on $\operatorname{End}_{d}^{N}$ is stable in the sense of geometric invariant theory.

Theorem 5.1. [Petsche-Szpiro-Tepper, Levy]

Let $N \geq 1$ and $d \geq 2$.

(a) For all geometric points $f \in \operatorname{End}_{d}^{N}$, the group Aut $(f)$ is finite. 
(b) The scheme $\operatorname{End}_{d}^{N}$ is contained in the $\mathrm{SL}_{N+1}$-stable locus of $\mathbb{P}^{M}$ relative to the invertible sheaf $\mathcal{O}_{\mathbb{P}^{M}}(1)$. The GIT quotient is typically denoted by $\mathcal{M}_{d}^{N}:=\operatorname{End}_{d}^{N} / / \mathrm{SL}_{N+1}$.

Proof. (a) Finiteness is proven in both [38] and [51, but the former has an even stronger result, namely that there is a bound for \# Aut $(f)$ that depends only on $N$ and $d$. Levy further proves that for any finite group $G \neq 1$, the set

$$
\left\{f \in \operatorname{End}_{d}^{N}: \operatorname{Aut}(f) \text { has a subgroup that is isomorphic to } G\right\}
$$

is an $\mathrm{SL}_{N+1}$-invariant Zariski closed subscheme of $\operatorname{End}_{d}^{N}$, hence descends to a Zariski closed subscheme of $\mathcal{M}_{d}^{N}$.

(b) Semi-stability of $\operatorname{End}_{d}^{N}$ is immediate from the definition, since the Macaulay resultant $\mathcal{R} \in \Gamma\left(\mathbb{P}^{M}, \mathcal{O}_{\mathbb{P}^{M}}\left((N+1) d^{N}\right)\right)$ is a global section whose non-vanishing defines $\operatorname{End}_{d}^{N}$, so $\operatorname{End}_{d}^{N}=\{\mathcal{R} \neq 0\}$ is an affine variety. (See [59, Theorem 1.8] for information about the Macaulay resultant.)

Stability of $\operatorname{End}_{d}^{N}$ is more delicate, the first step always being to prove that $\operatorname{Aut}(f)$ is finite. The original proof of stability for $\operatorname{End}_{d}^{1}$ is in [57], and there are two proofs in the literature for $\operatorname{End}_{d}^{N}$. The first, by Petsche-Szpiro-Tepper [51, is very direct. They apply [50, argument on page 10] to deduce that the action of $\mathrm{SL}_{N+1}$ in $\operatorname{End}_{d}^{N}$ is closed, after which they cite [50, Amplification 1.3 on page 30] to deduce that the geometric quotient exists. This last deduction depends crucially on the fact that $\operatorname{End}_{d}^{N}$ is affine, which as noted earlier follows from the fact that $\operatorname{End}_{d}^{N}$ is the complement of a hypersurface in $\mathbb{P}^{M}$.

The proof by Levy [38] uses the numerical criterion (Theorem 4.3), so is somewhat more cumbersome, but it has a number of advantages. First, as Levy points out, it allows one to describe more accurately the full semi-stable and stable loci. Second, as we show in Section 8 , with some additional work Levy's method allows us to deal with non-affine schemes such as $\operatorname{End}_{d}^{N} \times\left(\mathbb{P}^{N}\right)^{n}$.

\section{Models of Portraits and Portrait Moduli Problems}

In this section we describe what it means for a map $f \in \operatorname{End}_{d}^{N}$ and a collection of points in $\mathbb{P}^{N}$ to be a model for the portrait $\mathcal{P}$. We then define the $\mathcal{P}$-moduli problem to be that of classifying the set of isomorphism classes of models. The first order of business is to recall the definition of the multiplicity of a map $f$ at a point $P$, which is required in order to deal with weighted portraits. Readers who are interested only in unweighted portraits may skip this definition. 
Definition 6.1. Let $(f, P) \in \operatorname{End}_{d}^{N}(k) \times \mathbb{P}^{N}(k)$ be a geometric point. The multiplicity of $f$ at $P$, denoted $e_{f}(P)$, is defined as follows: Let

$$
\begin{aligned}
\mathcal{O}_{P} & :=\text { the local ring of } \mathbb{P}^{N} \text { at } P, \\
\mathfrak{M}_{P} & :=\text { the maximal ideal of } \mathcal{O}_{P} .
\end{aligned}
$$

Write $f=\left[f_{0}, \ldots, f_{N}\right]$ using homogeneous polynomials of degree $d$, choose an index $j$ with $f_{j}(P) \neq 0$, and let $I_{f, P}$ be the ideal of $\mathcal{O}_{P}$ defined by

$$
I_{f, P}:=\left(\frac{f_{0}}{f_{j}}-\frac{f_{0}}{f_{j}}(P), \frac{f_{1}}{f_{j}}-\frac{f_{1}}{f_{j}}(P), \ldots, \frac{f_{N}}{f_{j}}-\frac{f_{N}}{f_{j}}(P)\right) \subseteq \mathfrak{M}_{P} .
$$

To see that the ideal $I_{f, P}$ is independent of the choice of $j$, we note that if $k$ is some other index with $f_{k}(P) \neq 0$, then we have the identity

$$
\begin{aligned}
& \frac{f_{\ell}}{f_{k}}-\frac{f_{\ell}}{f_{k}}(P) \\
& =\left[\left(\frac{f_{\ell}}{f_{j}}-\frac{f_{\ell}}{f_{j}}(P)\right) \cdot \frac{f_{k}}{f_{j}}(P)-\left(\frac{f_{k}}{f_{j}}-\frac{f_{k}}{f_{j}}(P)\right) \cdot \frac{f_{\ell}}{f_{j}}(P)\right] \cdot \frac{f_{j}}{f_{k}} \cdot \frac{f_{j}}{f_{k}}(P) .
\end{aligned}
$$

We thus obtain a well-defined quantity

$$
e_{f}(P):=\operatorname{dim}_{k} \mathcal{O}_{P} / I_{f, P}
$$

For basic properties of multiplicities, and alternative equivalent definitions in various settings, see for example [27, page 669] or [29, 30].

Remark 6.2. In dimension 1, i.e., for $N=1$, the quantity $e_{f}(P)$ is also called the ramification index of $f$ at $P$. If $e_{f}(P) \geq 2$, then dynamicists say that $P$ is a critical point and that $f(P)$ is a critical value, while algebraic geometers say that $P$ is a ramification point and $f(P)$ is a branch point. If $P \neq \infty$ and $f(P) \neq \infty$, then the definition of $e_{f}(P)$ simplifies to

$$
e_{f}(P)=\operatorname{ord}_{P}(f-f(P)) .
$$

Further, working over an algebraically closed field $k$ of characteristic 0 , we have the standard formulas

$$
\operatorname{deg}(f)=\sum_{Q \in f^{-1}(P)} e_{f}(Q) \text { and } 2 \operatorname{deg}(f)-2=\sum_{P \in \mathbb{P}^{1}(k)}\left(e_{f}(P)-1\right) .
$$

Definition 6.3. Let $\mathcal{P}=\left(\mathcal{V}^{\circ}, \mathcal{V}, \Phi, \epsilon\right)$ be a portrait, and let $k$ be an algebraically closed field. A model for $\mathcal{P}$ over $k$ (of dimension $N$ and degree $d)$ is a pair $(f, \gamma)$, where $f$ and $\gamma$ are maps

$$
f \in \operatorname{End}_{d}^{N}(k) \text { and } \gamma: \mathcal{V} \longleftrightarrow \mathbb{P}^{N}(k)
$$


such that the diagram

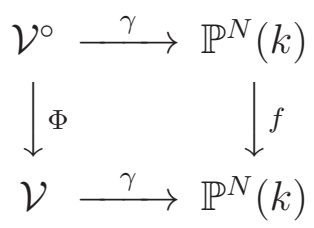

commutes, and such that the multiplicities of $f$ satisfy

$$
e_{f}(\gamma(v)) \geq \epsilon(v) \text { for all } v \in \mathcal{V}^{\circ} .
$$

We note that $\gamma$ is required to be injective, and also that if $\mathcal{P}$ is unweighted, then the multiplicity condition (6) is vacuous. We write $\operatorname{Model}_{d}^{N}[\mathcal{P}]$ for the set of pairs $(f, \gamma)$ satisfying (15) and (6).

Two models $(f, \gamma)$ and $\left(f^{\prime}, \gamma^{\prime}\right)$ are equivalent if there is a change of variables $\varphi \in \mathrm{PGL}_{N+1}(k)$ such that

$$
f^{\prime}=\varphi^{-1} \circ f \circ \varphi \text { and } \gamma^{\prime}=\varphi^{-1} \circ \gamma
$$

The $(N, d, \mathcal{P})$-moduli problem is to classify equivalence classes of $(N, d)$ models for $\mathcal{P}$.

Lemma 6.4. Let $\mathcal{P}_{1}$ and $\mathcal{P}_{2}$ be portraits such that $\operatorname{Model}_{d}^{N}\left(\mathcal{P}_{2}\right) \neq \emptyset$. There is a natural injective map

$$
\begin{aligned}
\operatorname{Hom}\left(\mathcal{P}_{1}, \mathcal{P}_{2}\right) & \longrightarrow \operatorname{Hom}\left(\operatorname{Model}_{d}^{N}\left(\mathcal{P}_{2}\right), \operatorname{Model}_{d}^{N}\left(\mathcal{P}_{1}\right)\right) \\
\alpha & \longmapsto((f, \gamma) \longmapsto(f, \gamma \circ \alpha)) .
\end{aligned}
$$

In other words, if $\alpha: \mathcal{P}_{1} \rightarrow \mathcal{P}_{2}$ is a portrait morphism as described in Definition 6.3 and if $(f, \gamma)$ is a model for $\mathcal{P}_{2}$, then $(f, \gamma \circ \alpha)$ is a model for $\mathcal{P}_{1}$.

Proof. Let $\alpha: \mathcal{P}_{1} \rightarrow \mathcal{P}_{2}$ be a portrait morphism and let $(f, \gamma)$ be a model for $\mathcal{P}_{2}$. The fact that $(f, \gamma \circ \alpha)$ is a model for $\mathcal{P}_{1}$ is immediate from the following facts, all of which come directly from the definitions:

(1) $\Phi\left(\mathcal{V}_{1}^{\circ}\right) \subseteq \mathcal{V}_{2}^{\circ}$

(2) $\alpha: \mathcal{V}_{1} \hookrightarrow \mathcal{V}_{2}$ and $\gamma: \mathcal{V} \hookrightarrow \mathbb{P}^{N}$ are injective.

(3) The diagram

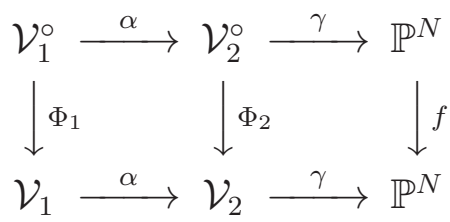

has commutative squares. 
(4) The weights behave properly, as we see from the following calculation:

$$
\begin{array}{cl}
e_{f} \circ \gamma \geq \epsilon_{2} & \text { since }(f, \gamma) \text { is a model for } \mathcal{P}_{2}, \\
e_{f} \circ \gamma \circ \alpha \geq \epsilon_{2} \circ \alpha & \text { composing with } \alpha, \\
e_{f} \circ \gamma \circ \alpha \geq \epsilon_{1} & \text { since } \epsilon_{2} \circ \alpha \geq \epsilon_{1} \text { by definition of portrait } \\
& \text { morphism (Definition 3.4). }
\end{array}
$$

Hence $(f, \gamma \circ \alpha)$ is a model for $\mathcal{P}_{1}$.

Finally, let $\alpha^{\prime}: \mathcal{P}_{1} \rightarrow \mathcal{P}_{2}$ be another portrait morphism, and suppose that $(f, \gamma \circ \alpha)=\left(f, \gamma \circ \alpha^{\prime}\right)$ for every model $(f, \gamma)$ of $\mathcal{P}_{2}$. This means that $\gamma \circ \alpha=\gamma \circ \alpha^{\prime}$, and then the assumed injectivity of $\gamma, \alpha$, and $\alpha^{\prime}$ forces $\alpha=\alpha^{\prime}$.

\section{The Parameter Space $\operatorname{End}_{d}^{N}[\mathcal{P}]$ for Models of a Portrait}

Our goal in this section is to construct a universal parameter space for the set of models of $\mathcal{P}$. We start with a bit of useful notation and a definition.

Definition 7.1. For any finite index set $\mathcal{N}$ and any scheme $S$, we write

$$
S^{\mathcal{N}}:=\prod_{n \in \mathcal{N}} S_{n} \quad \text { with each } S_{n} \cong S
$$

for the product of $\# \mathcal{N}$ copies of $S$, indexed by $\mathcal{N}$. For $n \in \mathcal{N}$, we let

$$
\pi_{S, n}: S^{\mathcal{N}} \longrightarrow S_{n} \cong S
$$

denote the projection map. If the scheme $S$ is clear from context, we may omit $S$ from the notation and write $\pi_{n}$.

Definition 7.2. Let $\mathcal{P}$ be a portrait. A universal parameter space for $(N, d)$-models of $\mathcal{P}$ is a scheme $E$ with the following property: Let $T$ be a scheme, let $f: T \rightarrow \operatorname{End}_{d}^{N}$ be a morphism, and let $\gamma: T \rightarrow\left(\mathbb{P}^{N}\right)^{\mathcal{V}}$ be a morphism such that for every geometric point $t \in T(k)$, the pair $\left(f_{t}, \gamma_{t}\right)$ is a model for $\mathcal{P}$ over $k$. Then there is a unique morphism $F: T \rightarrow E$ such that $F_{t}=\left(f_{t}, \gamma_{t}\right)$ for all geometric points $t \in T(k)$.

The construction of the universal parameter space, which we denote by $\operatorname{End}_{d}^{N}[\mathcal{P}]$, proceeds in two steps. We start with the case that $\mathcal{P}$ is an unweighted portrait. We then construct $\operatorname{End}_{d}^{N}[\mathcal{P}]$ for general $\mathcal{P}$ by taking a closed subscheme of the space for the associated unweighted portrait. 
7.1. The Parameter Space $\operatorname{End}_{d}^{N}[\mathcal{P}]$ for Unweighted Portraits. In this section we assume that $\mathcal{P}$ is an unweighted portrait. We construct a scheme $\operatorname{End}_{d}^{N}[\mathcal{P}]$, which we prove is a universal parameter space for $(N, d)$-models of $\mathcal{P}$. We start with $\operatorname{End}_{d}^{N}$, the parameter space of degree $d$ endomorphisms $f: \mathbb{P}^{N} \rightarrow \mathbb{P}^{N}$, as described in Section 5. In particular, $\operatorname{End}_{d}^{N}$ is identified with an affine subscheme of $\mathbb{P}^{M}$ by listing the coefficients of the homogeneous polynomials that define an endomorphism.

We recall that $\left(\mathbb{P}^{N}\right)^{\mathcal{V}}$ is a product of copies of $\mathbb{P}^{N}$ indexed by $\mathcal{V}$, and that for each $v \in \mathcal{V}$, we have a projection map $\pi_{v}:\left(\mathbb{P}^{N}\right)^{\mathcal{V}} \rightarrow \mathbb{P}^{N}$. For each pair of vertices $v, v^{\prime} \in \mathcal{V}$, we similarly have a bi-projection map

$$
\pi_{v} \times \pi_{v^{\prime}}:\left(\mathbb{P}^{N}\right)^{\mathcal{V}} \longrightarrow \mathbb{P}^{N} \times \mathbb{P}^{N} \text {. }
$$

We let

$$
\Delta \subset \mathbb{P}^{N} \times \mathbb{P}^{N}
$$

denote the diagonal, and then we define the complement of the big diagonal of $\left(\mathbb{P}^{N}\right)^{\mathcal{V}}$ to be the scheme

$$
\left(\mathbb{P}^{N}\right)_{\Delta}^{\mathcal{V}}:=\left(\mathbb{P}^{N}\right)^{\mathcal{V}} \backslash \bigcup_{v, v^{\prime} \in \mathcal{V}, v \neq v^{\prime}} \pi_{v, v^{\prime}}^{-1}(\Delta) .
$$

For example, if $k$ is an algebraically closed field, then $\left(\mathbb{P}^{N}\right)_{\Delta}^{\nu}(k)$ is isomorphic to the usual complement of the big diagonal of $\left(\mathbb{P}^{N}(k)\right)^{\# \mathcal{V}}$, i.e., $\left(\mathbb{P}^{N}\right)_{\Delta}^{\mathcal{V}}(k)$ is the set of $\# \mathcal{V}$-tuples of distinct points in $\mathbb{P}^{N}(k)$.

We observe that $\operatorname{End}_{d}^{N}$ and $\left(\mathbb{P}^{N}\right)_{\Delta}^{\mathcal{V}}$ are naturally quasi-projective schemes over $\operatorname{Spec}(\mathbb{Z})$, since they are open subschemes, respectively, of the schemes $\mathbb{P}^{M}$ and $\left(\mathbb{P}^{N}\right)^{\# \mathcal{V}}$. We want to define $\operatorname{End}_{d}^{N}[\mathcal{P}]$ to be the subscheme of $\operatorname{End}_{d}^{N} \times\left(\mathbb{P}^{N}\right)_{\Delta}^{\mathcal{V}}$ that consists of maps and points that are "models of $\mathcal{P}$."

We start with a map $G$ that we define at the level of geometric points by

$$
G: \operatorname{End}_{d}^{N} \times\left(\mathbb{P}^{N}\right)^{\mathcal{V}} \longrightarrow\left(\mathbb{P}^{N}\right)^{\mathcal{V}} \times\left(\mathbb{P}^{N}\right)^{\mathcal{V}}, \quad G(f, \boldsymbol{P})=(f(\boldsymbol{P}), \boldsymbol{P}) .
$$

More formally, let

$$
f: \operatorname{End}_{d}^{N} \times \mathbb{P}^{N} \rightarrow \mathbb{P}^{N}
$$

be the map giving the natural action of $\operatorname{End}_{d}^{N}$ on $\mathbb{P}^{N}$, i.e., at the level of geometric points $\boldsymbol{f}$ sends $(f, P) \rightarrow f(P)$. Then the map $G$ is the composition

$$
\begin{aligned}
G: \operatorname{End}_{d}^{N} \times\left(\mathbb{P}^{N}\right)^{\mathcal{V}} \stackrel{\operatorname{diag} \times \operatorname{diag}}{\longrightarrow}\left(\operatorname{End}_{d}^{N}\right)^{\mathcal{V}} \times\left(\left(\mathbb{P}^{N}\right)^{\mathcal{V}} \times\left(\mathbb{P}^{N}\right)^{\mathcal{V}}\right) \\
\stackrel{\sim}{\sim}\left(\operatorname{End}_{d}^{N} \times \mathbb{P}^{N}\right)^{\mathcal{V}} \times\left(\mathbb{P}^{N}\right)^{\mathcal{V}} \\
\stackrel{\boldsymbol{f}^{\mathcal{i d}}}{\longrightarrow}\left(\mathbb{P}^{N}\right)^{\mathcal{V}} \times\left(\mathbb{P}^{N}\right)^{\mathcal{V}} .
\end{aligned}
$$


Next, for each pair of vertices $v, v^{\prime} \in \mathcal{V}$, we define a map

$$
\Psi_{v, v^{\prime}}: \operatorname{End}_{d}^{N} \times\left(\mathbb{P}^{N}\right)_{\Delta}^{\mathcal{V}} \rightarrow \mathbb{P}^{N} \times \mathbb{P}^{N}, \quad \Psi_{v, v^{\prime}}=\left(\pi_{v} \times \pi_{v^{\prime}}\right) \circ G
$$

that first applies $G$ and then projects onto the indicated factors. Recalling that $\Delta$ denotes the diagonal of $\mathbb{P}^{N} \times \mathbb{P}^{N}$, we define $\operatorname{End}_{d}^{N}[\mathcal{P}]$ to be the scheme

$$
\operatorname{End}_{d}^{N}[\mathcal{P}]:=\bigcap_{v \in \mathcal{V}^{\circ}} \Psi_{v, \Phi(v)}^{-1}(\Delta)
$$

To trace out what this means, let

$$
\left(f,\left(P_{w}\right)_{w \in \mathcal{V}}\right) \in \operatorname{End}_{d}^{N}(k) \times\left(\mathbb{P}^{N}\right)_{\Delta}^{\mathcal{V}}(k)
$$

be a geometric point, where we interpret the second coordinate as being given by an injective map $P: \mathcal{V} \hookrightarrow \mathbb{P}^{N}(k)$. (The injectivity comes from the fact that we are required to be in $\left(\mathbb{P}^{N}\right)_{\Delta}^{\mathcal{V}}(k)$, which is the complement of the big diagonal.) Then

$$
\begin{aligned}
\left(f,\left(P_{w}\right)_{w \in \mathcal{V}}\right) \in & \operatorname{End}_{d}^{N}[\mathcal{P}](k) \\
& \Longleftrightarrow \Psi_{v, \Phi(v)}\left(f,\left(P_{w}\right)_{w \in \mathcal{V}}\right) \in \Delta \quad \text { for all } v \in \mathcal{V}^{\circ} .
\end{aligned}
$$

We compute

$$
\begin{array}{rlrl}
\Psi_{v, \Phi(v)}(f, & \left.\left(P_{w}\right)_{w \in \mathcal{V}}\right) & \\
& =\left(\pi_{v} \times \pi_{\Phi(v)}\right) \circ G\left(f,\left(P_{w}\right)_{w \in \mathcal{V}}\right) & & \text { definition of } \Psi_{v, v^{\prime}} \\
& =\left(\pi_{v} \times \pi_{\Phi(v)}\right)\left(\left(f\left(P_{w}\right)\right)_{w \in \mathcal{V}},\left(P_{w}\right)_{w \in \mathcal{V}}\right) & & \text { definition of } G \\
& =\left(f\left(P_{v}\right), P_{\Phi(v)}\right) & & \text { definition of } \pi_{v} .
\end{array}
$$

Hence

$$
\left(f,\left(P_{w}\right)_{w \in \mathcal{V}}\right) \in \operatorname{End}_{d}^{N}[\mathcal{P}](k) \Longleftrightarrow f\left(P_{v}\right)=P_{\Phi(v)} \text { for all } v \in \mathcal{V}^{\circ},
$$

which says exactly that $(f, P)$ is a model for $\mathcal{P}$.

Proposition 7.3. Let $\mathcal{P}$ be an unweighted portrait, and let

$$
\operatorname{End}_{d}^{N}[\mathcal{P}] \subset \operatorname{End}_{d}^{N} \times\left(\mathbb{P}^{N}\right)^{\mathcal{V}} \subset \mathbb{P}^{M} \times\left(\mathbb{P}^{N}\right)^{\mathcal{V}}
$$

be the scheme described by (8).

(a) $\operatorname{End}_{d}^{N}[\mathcal{P}]$ is a universal parameter space for models of $\mathcal{P}$.

(b) $\operatorname{End}_{d}^{N}[\mathcal{P}]$ is an $\mathrm{SL}_{N+1}$-invariant subvariety of $\mathbb{P}^{M} \times\left(\mathbb{P}^{N}\right)^{\mathcal{V}}$.

Proof. (a) Let $T$ be a scheme, and let

$$
f: T \rightarrow \operatorname{End}_{d}^{N} \quad \text { and } \quad \gamma: T \rightarrow\left(\mathbb{P}^{N}\right)^{\mathcal{V}}
$$

be morphisms such that for every geometric point $t \in T(k)$, the pair $(f(t), \gamma(t)))$ is a model for $\mathcal{P}$ over $k$. We need to show that there is a unique morphism $F: T \rightarrow \operatorname{End}_{d}^{N}[\mathcal{P}]$ such that $\left.F(t)=(f(t), \gamma(t))\right)$ for 
all geometric points $t \in T(k)$. The given $f$ and $\gamma$ define a morphism $(f, \gamma): T \rightarrow \operatorname{End}_{d}^{N} \times\left(\mathbb{P}^{N}\right)^{\mathcal{V}}$, and (9) combined with the assumptions on $f$ and $\gamma$ imply that the image of this morphism lies in the subscheme $\operatorname{End}_{d}^{N}[\mathcal{P}]$.

(b) We need to verify that $\operatorname{End}_{d}^{N}[\mathcal{P}]$ is an $\mathrm{SL}_{N+1}$-invariant subscheme of $\mathbb{P}^{M} \times\left(\mathbb{P}^{N}\right)^{\mathcal{V}}$, so first we need to describe the action. We let $\varphi \in$ $\mathrm{SL}_{N+1}$ act on $f \in \operatorname{End}_{d}^{N}$ by conjugation $f^{\varphi}:=\varphi^{-1} \circ f \circ \varphi$ and we extend this action to $\mathbb{P}^{M}$ as usual. Similarly, we let $\varphi \in \mathrm{SL}_{N+1}$ act on $\left(P_{v}\right) \in\left(\mathbb{P}^{N}\right)^{\mathcal{V}}$ via the diagonal action $\left(P_{v}\right)^{\varphi}:=\left(\varphi^{-1}\left(P_{v}\right)\right)$. We observe that $\left(\mathbb{P}^{N}\right)_{\Delta}^{\mathcal{V}}$ is an $\mathrm{SL}_{N+1}$-invariant subscheme of $\left(\mathbb{P}^{N}\right)^{\mathcal{V}}$, since the big diagonal is $\mathrm{SL}_{N+1}$-invariant.

It suffices to check the $\mathrm{SL}_{N+1}$-invariance on geometric points, so we need to show that

$$
\begin{aligned}
\left(f,\left(P_{w}\right)_{w \in \mathcal{V}}\right) \in \operatorname{End}_{d}^{N}[\mathcal{P}](k) & \text { and } \varphi \in \operatorname{SL}_{N+1}(k) \\
& \Longrightarrow \quad\left(f^{\varphi},\left(P_{w}^{\varphi}\right)_{w \in \mathcal{V}}\right) \in \operatorname{End}_{d}^{N}[\mathcal{P}](k) .
\end{aligned}
$$

Applying the characterization (9) of geometric points in $\operatorname{End}_{d}^{N}[\mathcal{P}]$, we need to show that

$$
f\left(P_{v}\right)=P_{\Phi(v)} \text { for all } v \in \mathcal{V}^{\circ} \Longrightarrow f^{\varphi}\left(P_{v}^{\varphi}\right)=P_{\Phi(v)}^{\varphi} \text { for all } v \in \mathcal{V}^{\circ} .
$$

But the definitions have been set up so that for all $v \in \mathcal{V}^{\circ}$,

$$
f^{\varphi}\left(P_{v}^{\varphi}\right)=\left(\varphi^{-1} \circ f \circ \varphi\right)\left(\varphi^{-1}\left(P_{v}\right)\right)=\varphi^{-1}\left(f\left(P_{v}\right)\right)=\varphi^{-1}\left(P_{\Phi(v)}\right)=P_{\Phi(v)}^{\varphi} .
$$

This completes the verification that $\operatorname{End}_{d}^{N}[\mathcal{P}]$ is $\mathrm{SL}_{N+1}$-invariant.

Remark 7.4. We describe $\operatorname{End}_{d}^{N}[\mathcal{P}]$ more explicitly. For convenience, we let $\mathcal{V}=\{1,2, \ldots, n\}$ with $\mathcal{V}^{\circ}=\{1,2, \ldots, m\}$. (Note that if $\mathcal{P}=\emptyset$ is the empty portrait, then $\operatorname{End}_{d}^{N}[\mathcal{P}]=\operatorname{End}_{d}^{N}$.) We start with the scheme

$$
\left(\mathbb{P}^{N}\right)^{n}:=\prod_{i=1}^{n} \operatorname{Proj} \mathbb{Z}\left[X_{0}^{(i)}, X_{1}^{(i)}, \ldots, X_{N}^{(i)}\right] .
$$

For each $i \neq j$, the diagonal $\Delta_{i, j}$ corresponding to the condition $P_{i}=P_{j}$ is the subscheme described by the equations

$$
\Delta_{i, j}:=\left(X_{k}^{(j)} X_{\ell}^{(i)}-X_{\ell}^{(j)} X_{k}^{(i)}\right)_{0 \leq k, \ell \leq N} .
$$

We let

$$
\left(\mathbb{P}^{N}\right)_{\Delta}^{n}=\left(\mathbb{P}^{N}\right)^{n} \backslash \bigcup_{1 \leq i<j \leq n} \Delta_{i, j}
$$

be the complement of the big diagonal. 
Similarly, for each $i$ and $j$, the condition $f\left(P_{i}\right)=P_{j}$ defines a closed subscheme $\Gamma_{i, j}$ of $\operatorname{End}_{d}^{N} \times\left(\mathbb{P}^{N}\right)_{\Delta}^{n}$. Explicitly, if we write $f=$ $\left[f_{0}, \ldots, f_{N}\right]$, then $\Gamma_{i, j}$ is the subscheme described by the equations

$$
\Gamma_{i, j}:=\left(X_{k}^{(j)} f_{\ell}\left(X_{0}^{(i)}, \ldots, X_{N}^{(i)}\right)-X_{\ell}^{(j)} f_{k}\left(X_{0}^{(i)}, \ldots, X_{N}^{(i)}\right)\right)_{0 \leq k, \ell \leq N} .
$$

Finally, we can describe $\operatorname{End}_{d}^{N}[\mathcal{P}]$ as the intersection

$$
\operatorname{End}_{d}^{N}[\mathcal{P}]:=\bigcap_{i=1}^{m} \Gamma_{i, \Phi(i)} .
$$

Remark 7.5. There are certain natural geometric constraints that $\mathcal{P}$ must satisfy in order for $\operatorname{End}_{d}^{N}[\mathcal{P}]$ to be non-empty. See Theorem 14.2 for unweighted portraits, and also Proposition 15.1 for weighted portraits when $N=1$.

Remark 7.6. We have constructed $\operatorname{End}_{d}^{N}[\mathcal{P}]$ as a subscheme of $\mathbb{P}^{M} \times$ $\left(\mathbb{P}^{N}\right)^{\mathcal{V}}$. In practice, it is often possible to specify the portrait $\mathcal{P}$ using fewer than $\# \mathcal{V}$ points. This, in turn, can be used to give a simpler description of $\mathcal{M}_{d}^{N}[\mathcal{P}]$, i.e., a description using fewer equations in a lower-dimensional ambient space. This is the approach taken by the first author in [16], where dynamical modular curves are constructed for polynomials $x^{2}+c$ with portraits $\mathcal{P}$ specified by generating sets. However, there are subtleties in this approach, since disconnectedness of $\mathcal{P}$ may lead to extraneous components in the naively constructed moduli space. We refer the reader to [16, Example 2.16] for details.

7.2. The Parameter Space $\operatorname{End}_{d}^{N}[\mathcal{P}]$ for Weighted Portraits. In this section we construct a universal parameter space $\operatorname{End}_{d}^{N}[\mathcal{P}]$, where now $\mathcal{P}=\left(\mathcal{V}^{\circ}, \mathcal{V}, \Phi, \epsilon\right)$ is allowed to include non-trivial weights. We write $\mathcal{P}^{\prime}=\left(\mathcal{V}^{\circ}, \mathcal{V}, \Phi, \epsilon^{\prime}\right)$ for the associated unweighted portrait, i.e., $\mathcal{P}$ and $\mathcal{P}^{\prime}$ have the same vertices and the same directed edges, but $\epsilon^{\prime}(v)=$ 1 for all $v \in \mathcal{V}^{\circ}$. In Section 7.1 we constructed the space $\operatorname{End}_{d}^{N}\left[\mathcal{P}^{\prime}\right]$. In this section we show that we can take $\operatorname{End}_{d}^{N}[\mathcal{P}]$ to be a Zariski closed subscheme of $\operatorname{End}_{d}^{N}\left[\mathcal{P}^{\prime}\right]$.

Theorem 7.7. Let $\mathcal{P}$ be a portrait, let $\mathcal{P}^{\prime}$ be the associated unweighted portrait, and let $\operatorname{End}_{d}^{N}\left[\mathcal{P}^{\prime}\right]$ be the scheme described in Proposition 7.3 . There is a Zariski closed subscheme of $\operatorname{End}_{d}^{N}\left[\mathcal{P}^{\prime}\right]$, which we denote by $\operatorname{End}_{d}^{N}[\mathcal{P}]$, whose geometric points are specified by

$$
\operatorname{End}_{d}^{N}[\mathcal{P}]:=\left\{(f, \boldsymbol{P}) \in \operatorname{End}_{d}^{N}\left[\mathcal{P}^{\prime}\right]: e_{f}\left(P_{v}\right) \geq \epsilon(v) \text { for all } v \in \mathcal{V}^{\circ}\right\}
$$

The space $\operatorname{End}_{d}^{N}[\mathcal{P}]$ has the following properties: 
(a) $\operatorname{End}_{d}^{N}[\mathcal{P}]$ is a universal parameter space for models of $\mathcal{P}$.

(b) $\operatorname{End}_{d}^{N}[\mathcal{P}]$ is an $\mathrm{SL}_{N+1}$-invariant subvariety of $\operatorname{End}_{d}^{N}\left[\mathcal{P}^{\prime}\right]$.

Proposition 7.8. Let $\mathcal{P}_{1}$ and $\mathcal{P}_{2}$ be weighted portraits. There is a natural map

$$
\operatorname{Hom}\left(\mathcal{P}_{1}, \mathcal{P}_{2}\right) \longrightarrow \operatorname{Hom}\left(\operatorname{End}_{d}^{N}\left[\mathcal{P}_{2}\right], \operatorname{End}_{d}^{N}\left[\mathcal{P}_{1}\right]\right)
$$

induced by the map on models described in Lemma 6.4, i.e., a portrait morphism $\mathcal{P}_{1} \rightarrow \mathcal{P}_{2}$ induces a morphism $\operatorname{End}_{d}^{N}\left[\mathcal{P}_{2}\right] \rightarrow \operatorname{End}_{d}^{N}\left[\mathcal{P}_{1}\right]$ of schemes.

We start with the case of a single point.

Proposition 7.9. Let $\epsilon \geq 0$ be an integer. Then the locus

$$
\left\{(f, P) \in \operatorname{End}_{d}^{N} \times \mathbb{P}^{N}: e_{f}(P) \geq \epsilon\right\}
$$

is a Zariski closed subscheme of $\operatorname{End}_{d}^{N} \times \mathbb{P}^{N}$.

The key to proving Proposition 7.9 is the following lemma, which is used to show that the set (11) is defined by finitely many polynomial relations.

Lemma 7.10. With notation as in Definition 6.1, we have:

(a) $e_{f}(P)$ is finite.

(b) For all integers $\epsilon \geq 0$, we have

$$
e_{f}(P) \geq \operatorname{dim}_{k}\left(\frac{\mathcal{O}_{P}}{I_{f, P}+\mathfrak{M}_{P}^{\epsilon}}\right) \geq \min \left\{\epsilon, e_{f}(P)\right\} .
$$

Proof. (a) Let $j$ be an index for which $f_{j}(P) \neq 0$, and let $V_{f, P, j}$ be the Zariski closure in $\mathbb{P}^{N}$ of the set

$$
\left\{Q \in \mathbb{P}^{N}: f_{j}(Q) \neq 0 \text { and }\left(f_{i} / f_{j}\right)(Q)=\left(f_{i} / f_{j}\right)(P) \text { for all } 0 \leq i \leq N\right\} \text {. }
$$

Then $e_{f}(P)=\infty$ if and only if $V_{f, P, j}$ has dimension greater than 0 . But if $\operatorname{dim} V_{f, P, j} \geq 1$, then $V_{f, P, j} \cap\left\{f_{j}=0\right\} \neq \emptyset$, and points in this intersection are indeterminacy points of $f$.

(b) The first inequality is immediate from the definition of $e_{f}(P)$. To ease notation, let $M:=\mathcal{O}_{P} / I_{f, P}$, where we view $M$ as a finitely generated $\mathcal{O}_{P}$-module. Let $r \geq 0$ be the largest integer (or $\infty$ ) such that

$$
\mathfrak{M}_{P}^{i} M \neq \mathfrak{M}_{P}^{i+1} M \quad \text { for all } 0 \leq i<r .
$$

Then we have a descending chain of $\mathcal{O}_{P}$-modules,

$$
M \supsetneq \mathfrak{M}_{P} M \supsetneq \mathfrak{M}_{P}^{2} M \supsetneq \mathfrak{M}_{P}^{3} M \supsetneq \cdots \supsetneq \mathfrak{M}_{P}^{r} M=\mathfrak{M}_{P}^{r+1} M,
$$


and considering the successive quotients, we see that

$$
e_{f}(P):=\operatorname{dim}_{k} M=\sum_{i=0}^{\infty} \operatorname{dim}_{k}\left(\frac{\mathfrak{M}_{P}^{i} M}{\mathfrak{M}_{P}^{i+1} M}\right) \geq \sum_{i=0}^{r-1} 1=r .
$$

This proves that $r$ is finite, since we know from (a) that $e_{f}(P)$ is finite.

Further, our choice of $r$ implies that $\mathfrak{M}_{P} \cdot \mathfrak{M}_{P}^{r} M=\mathfrak{M}_{P}^{r} M$. Applying Nakayama's lemma to the finitely generated $\mathcal{O}_{P}$-module $\mathfrak{M}_{P}^{r} M$, we conclude that $\mathfrak{M}_{P}^{r} M=0$. Hence the chain (12) terminates with 0 , and we obtain an equality

$$
e_{f}(P):=\operatorname{dim}_{k} M=\sum_{i=0}^{r-1} \operatorname{dim}_{k}\left(\frac{\mathfrak{M}_{P}^{i} M}{\mathfrak{M}_{P}^{i+1} M}\right) .
$$

We use the isomorphisms

$$
\frac{\mathcal{O}_{P}}{I_{f, P}+\mathfrak{M}_{P}^{\epsilon}} \cong \frac{\mathcal{O}_{P} / I_{f, P}}{\left(I_{f, P}+\mathfrak{M}_{P}^{\epsilon}\right) / I_{f, P}} \cong \frac{\mathcal{O}_{P} / I_{f, P}}{\mathfrak{M}_{P}^{\epsilon} /\left(I_{f, P} \cap \mathfrak{M}_{P}^{\epsilon}\right)} \cong \frac{M}{\mathfrak{M}_{P}^{\epsilon} M}
$$

and the chain (12) to compute

$$
\operatorname{dim}_{k}\left(\frac{\mathcal{O}_{P}}{I_{f, P}+\mathfrak{M}_{P}^{\epsilon}}\right)=\operatorname{dim}_{k}\left(\frac{M}{\mathfrak{M}_{P}^{\epsilon} M}\right)=\sum_{i=0}^{\epsilon-1} \operatorname{dim}_{k}\left(\frac{\mathfrak{M}_{P}^{i} M}{\mathfrak{M}_{P}^{i+1} M}\right) .
$$

If $\epsilon \leq r-1$, then every term in the sum is at least 1 , so we get a lower bound of $\epsilon$ as desired. On the other hand, if $\epsilon \geq r$, then (13) tells us that the sum in (14) is equal to $e_{f}(P)$.

Proof of Proposition 7.9. We consider a polynomial ring

$$
\mathbb{Z}[\boldsymbol{a}, \boldsymbol{x}, \boldsymbol{u}]
$$

where $\boldsymbol{a}:=\left(a_{\rho}(\boldsymbol{e})\right)$ are the coefficients of $f_{0}, \ldots, f_{N}$ as described by (2), and where $\boldsymbol{x}=\left[x_{0}, \ldots, x_{N}\right]$ and $\boldsymbol{u}=\left[u_{0}, \ldots, u_{N}\right]$ are independent sets of coordinates on $\mathbb{P}^{N}$. The idea is that $\boldsymbol{x}$ is used for the variables in $f$, while $\boldsymbol{u}$ is a point at which we want to compute the multiplicity of $f$. We view $\mathbb{Z}[\boldsymbol{a}, \boldsymbol{x}, \boldsymbol{u}]$ as a trihomogeneous polynomial ring, so

$$
\operatorname{Proj} \mathbb{Z}[\boldsymbol{a}, \boldsymbol{x}, \boldsymbol{u}] \cong \mathbb{P}_{\boldsymbol{a}}^{M} \times \mathbb{P}_{\boldsymbol{x}}^{N} \times \mathbb{P}_{\boldsymbol{u}}^{N}
$$

where the subscripts indicate the homogeneous coordinates on the three factors.

We fix an integer $\epsilon \geq 0$ and want to describe the set of $(\boldsymbol{a}, \boldsymbol{x}, \boldsymbol{u})$ such that $f_{\boldsymbol{a}}(\boldsymbol{x})$ has multiplicity at least $\epsilon$ at $\boldsymbol{u}$. It suffices to do this on the open sets $x_{i} u_{j} \neq 0$ for all $0 \leq i, j \leq N$, and by symmetry it is enough to work on the open set $x_{0} u_{0} \neq 0$. So we dehomogenize by setting

$$
X_{i}:=x_{i} / x_{0} \quad \text { and } \quad U_{i}:=u_{i} / u_{0}
$$


and we let

$R:=\mathbb{Z}[\boldsymbol{a}, \boldsymbol{X}, \boldsymbol{U}]$ with $\operatorname{Proj} \mathbb{Z}[\boldsymbol{a}] \times \operatorname{Spec} \mathbb{Z}[\boldsymbol{X}, \boldsymbol{U}] \cong \mathbb{P}_{\boldsymbol{a}}^{M} \times \mathbb{A}_{\boldsymbol{X}}^{N} \times \mathbb{A}_{\boldsymbol{U}}^{N}$.

We consider the prime ideal $\mathfrak{M}$ of $R$ defined by

$$
\mathfrak{M}:=\left(X_{1}-U_{1}, X_{2}-U_{2}, \ldots, X_{N}-U_{N}\right) \subset R .
$$

We let $R_{\boldsymbol{U}}$ be the localization of $R$ at $\mathfrak{M}$,

$$
R_{U}:=\{A / B: A \in R, B \in R \backslash \mathfrak{M}\},
$$

we let $\mathfrak{M}_{U}$ be its maximal ideal,

$$
\mathfrak{M}_{U}:=\{A / B: A \in \mathfrak{M}, B \in R \backslash \mathfrak{M}\},
$$

and we define an ideal $I_{\boldsymbol{U}, f}$ in $R_{\boldsymbol{U}}$ by ${ }^{3}$

$$
I_{\boldsymbol{U}, f}=\left(f_{i}(\boldsymbol{X}) f_{j}(\boldsymbol{U})-f_{j}(\boldsymbol{X}) f_{i}(\boldsymbol{U})\right)_{1 \leq i, j \leq N^{*}}
$$

We note that $I_{\boldsymbol{U}, f} \subseteq \mathfrak{M}_{\boldsymbol{U}}$, since the functions in $I_{\boldsymbol{U}, f}$ vanish at $\boldsymbol{X}=\boldsymbol{U}$.

If we take particular values for $\boldsymbol{a}$ and $\boldsymbol{U}$ defined over an algebraically closed field $k$, i.e., if we take a particular $f \in \operatorname{End}_{d}^{N}(k)$ and a particular point $P \in \mathbb{P}^{N}(k)$, then unsorting the definitions, we see that

$$
e_{f}(P)=\operatorname{dim}_{k} R_{\boldsymbol{U}(P)} / I_{\boldsymbol{U}(P), f} .
$$

Further, Lemma 7.10 tells us that for any integer $\epsilon \geq 0$, we have

$$
e_{f}(P) \geq \epsilon \quad \Longleftrightarrow \operatorname{dim}_{k} R_{\boldsymbol{U}(P)} /\left(I_{\boldsymbol{U}(P), f}+\mathfrak{M}_{\boldsymbol{U}(P)}^{\epsilon}\right) \geq \epsilon .
$$

We want to turn this around and describe a Zariski closed condition on $(\boldsymbol{a}, \boldsymbol{U})$ that corresponds to requiring $e_{f}(P) \geq \epsilon$. We note that (15) tells us that it suffices to work modulo the ideal $\mathfrak{M}_{U}^{\epsilon}$, and that the quotient ring $R_{\boldsymbol{U}} / \mathfrak{M}_{\boldsymbol{U}}^{\epsilon}$ is a free $\mathbb{Z}[\boldsymbol{a}, \boldsymbol{U}]$-module with basis given by the following finite set of polynomials:

$$
\mathcal{S}:=\left\{\left(X_{1}-U_{1}\right)^{\delta_{1}}\left(X_{2}-U_{2}\right)^{\delta_{2}} \cdots\left(X_{N}-U_{N}\right)^{\delta_{N}}: \delta_{1}+\cdots+\delta_{N}<\epsilon\right\} .
$$

From (15), we see that we need to describe the locus where

$$
\operatorname{rank}_{\mathbb{Z}[\boldsymbol{a}, \boldsymbol{U}]} R_{\boldsymbol{U}} /\left(I_{\boldsymbol{U}, f}+\mathfrak{M}_{\boldsymbol{U}}^{\epsilon}\right) \geq \epsilon .
$$

To ease notation, we let

$n:=\# \mathcal{S}, \quad A:=\mathbb{Z}[\boldsymbol{a}, \boldsymbol{U}], \quad \bar{R}:=R_{\boldsymbol{U}} / \mathfrak{M}_{\boldsymbol{U}}^{\epsilon}, \quad \bar{R}^{\prime}:=R_{\boldsymbol{U}} /\left(I_{\boldsymbol{U}, f}+\mathfrak{M}_{\boldsymbol{U}}^{\epsilon}\right)$.

As noted earlier, the ring $\bar{R}$ is a free $A$-module with basis $\mathcal{S}$, so $n=$ $\operatorname{rank}_{A} \bar{R}$. To form $\bar{R}^{\prime}$, we take the quotient of $\bar{R}$ by the elements of the ideal $I_{\boldsymbol{U}, f}$, where $I_{\boldsymbol{U}, f}$ is generated as an $R_{\boldsymbol{U}}$-module by the finitely many polynomials $f_{i}(\boldsymbol{X}) f_{j}(\boldsymbol{U})-f_{j}(\boldsymbol{X}) f_{i}(\boldsymbol{U})$. And although the rank of $I_{\boldsymbol{U}, f}$ as an $A$-module is infinite, since we are also taking the quotient

\footnotetext{
${ }^{3}$ The polynomial $f_{i}$ is bihomogeneous of bidegree $(1, d)$ in the variables $\boldsymbol{a}$ and $\boldsymbol{x}$, and the notation $f_{i}(\boldsymbol{X})$ is shorthand for $f_{i}(\boldsymbol{X}):=x_{0}^{-d} f_{i}(\boldsymbol{x})=f_{i}\left(1, X_{1}, \ldots, X_{N}\right)$.
} 
by $\mathfrak{M}_{\boldsymbol{U}}^{\epsilon}$, it suffices to reduce the elements in $I_{f, \boldsymbol{U}}$ modulo $\mathfrak{M}_{\boldsymbol{U}}^{\epsilon}$ and thereby express them as $A$-linear combinations of elements of $\mathcal{S}$.

Explicitly, for each $i, j \in\{1, \ldots, N\}$ and each $h(\boldsymbol{X}, \boldsymbol{U}) \in \mathcal{S}$, we multiply the $(i, j)$ generator of $I_{f, P}$ by $h$, reduce modulo $\mathfrak{M}_{\boldsymbol{U}}^{\epsilon}$, and write the result as an $A$-linear combination of the basis $\mathcal{S}$. Thus

$$
\begin{aligned}
&\left(f_{i}(\boldsymbol{X}) f_{j}(\boldsymbol{U})\right.\left.-f_{j}(\boldsymbol{X}) f_{i}(\boldsymbol{U})\right) h(\boldsymbol{X}, \boldsymbol{U}) \\
& \equiv \sum_{g(\boldsymbol{X}, \boldsymbol{U}) \in \mathcal{S}} \alpha_{g}(i, j, h) g(\boldsymbol{X}, \boldsymbol{U}) \quad\left(\bmod \mathfrak{M}_{\boldsymbol{U}}^{\epsilon}\right) \\
& \text { with } \alpha_{g}(i, j, h) \in A
\end{aligned}
$$

Then as an $A$-module, the ring $\bar{R}^{\prime}$ is generated by the elements of $\mathcal{S}$, and the relations among those elements are exactly those described by (16).

We define $M$ to be a matrix with columns indexed by $g \in \mathcal{S}$, with rows indexed by triples $(i, j, h)$ with $1 \leq i, j \leq n$ and $h \in \mathcal{S}$. We set the $g \times(i, j, h)$ entry of $M$ to be $\alpha_{g}(i, \bar{j}, h)$. Then $M$ is an $N^{2} n$-by-n matrix with coefficients in $A$.

We are identifying $A^{n}$ with $A^{\mathcal{S}}$, where an element $\boldsymbol{a} \in A^{\mathcal{S}}$ is a map $\boldsymbol{a}: \mathcal{S} \rightarrow A$, and the map $A^{\mathcal{S}} \rightarrow \bar{R}^{\prime}$ is given by

$$
A^{\mathcal{S}} \rightarrow \bar{R}^{\prime}, \quad \boldsymbol{a} \longmapsto \sum_{g(\boldsymbol{X}, \boldsymbol{U}) \in \mathcal{S}} \boldsymbol{a}_{g} \cdot g(\boldsymbol{X}, \boldsymbol{U}) \quad\left(\bmod I_{\boldsymbol{U}, f}+\mathfrak{M}_{\boldsymbol{U}}^{\epsilon}\right) .
$$

The kernel of this map is generated by the relations (16), where there is one relation for each choice of $(i, j, h)$. Hence the kernel is generated by the row-span of $M$, i.e., by $A^{N^{2} n} M$. This gives the isomorphism

$$
\bar{R}^{\prime} \cong A^{n} / A^{N^{2} n} M,
$$

so in particular we have

$$
\operatorname{rank}_{\mathbb{Z}[\boldsymbol{a}, \boldsymbol{U}]} R_{\boldsymbol{U}} /\left(I_{\boldsymbol{U}, f}+\mathfrak{M}_{\boldsymbol{U}}^{\epsilon}\right)=\operatorname{rank}_{A} \bar{R}^{\prime}=\operatorname{rank}_{A} A^{n} / A^{N^{2} n} M .
$$

This yields

$$
\operatorname{rank}_{\mathbb{Z}[\boldsymbol{a}, \boldsymbol{U}]} R_{\boldsymbol{U}} /\left(I_{\boldsymbol{U}, f}+\mathfrak{M}_{\boldsymbol{U}}^{\epsilon}\right) \geq \epsilon \Longleftrightarrow \operatorname{rank}_{A} M \leq n-\epsilon,
$$

where $\operatorname{rank}_{A} M$ denotes the row-rank, or equivalently the column rank, of the matrix $M$ as an $A$-module.

Finally, we observe that $\operatorname{rank}_{A} M \leq n-\epsilon$ if and only if all of the $n-\epsilon+1$ square minors of $M$ have determinant equal to 0 . Each of these determinantal equations defines a Zariski closed set, and their intersection is the required locus in $\operatorname{End}_{d}^{N} \times \mathbb{P}^{N}$. This completes the proof of Proposition 7.9 . 
Remark 7.11. For a given $f \in \operatorname{End}_{d}^{N}$, the condition $e_{f}(P) \geq 2$ is a codimension 1 condition, since

$$
\left\{P \in \mathbb{P}^{N}: e_{f}(P) \geq 2\right\}=\operatorname{Crit}(f)
$$

is a subvariety of codimension 1 and degree $(N+1)(d-1)$. More generally for $\epsilon \geq 3$, it seems interesting to study the subscheme $\{P \in$ $\left.\mathbb{P}^{N}: e_{f}(P) \geq \epsilon\right\}$ and to understand how it varies with $f$.

Proof of Theorem 7.7. The scheme $\operatorname{End}_{d}^{N}\left[\mathcal{P}^{\prime}\right]$ as constructed in Proposition 7.3 is a closed subscheme of $\operatorname{End}_{d}^{N} \times\left(\mathbb{P}^{N}\right)^{\mathcal{V}}$, which is in turn an open subscheme of $\mathbb{P}^{M} \times\left(\mathbb{P}^{N}\right)^{\mathcal{V}}$. For each $v \in \mathcal{V}^{\circ}$, we consider the projection map

$$
\left(\mathrm{id} \times \pi_{v}\right): \operatorname{End}_{d}^{N} \times\left(\mathbb{P}^{N}\right)^{\mathcal{V}} \longrightarrow \operatorname{End}_{d}^{N} \times \mathbb{P}^{N}
$$

and we define $\operatorname{End}_{d}^{N}[\mathcal{P}]$ to be the intersection of the inverse images

$$
\begin{aligned}
& \operatorname{End}_{d}^{N}[\mathcal{P}]:=\operatorname{End}_{d}^{N}\left[\mathcal{P}^{\prime}\right] \\
& \cap \bigcap_{v \in \mathcal{V}^{\circ}}\left(1 \times \pi_{v}\right)^{-1}\left\{(f, \boldsymbol{P}) \in \operatorname{End}_{d}^{N} \times \mathbb{P}^{N}: e_{f}\left(P_{v}\right) \geq \epsilon(v)\right\} .
\end{aligned}
$$

Proposition 7.9 tells us that the individual sets in the intersection (17) are closed subschemes of $\operatorname{End}_{d}^{N} \times \mathbb{P}^{N}$, so their pull-backs by the projection maps are closed, as is their intersection. Since $\operatorname{End}_{d}^{N}\left[\mathcal{P}^{\prime}\right]$ is also a closed subscheme of $\operatorname{End}_{d}^{N} \times \mathbb{P}^{N}$, the same is true of $\operatorname{End}_{d}^{N}[\mathcal{P}]$.

(a) Let $(T, f, \gamma)$ be as in the definition 7.2 of universal parameter space. Ignoring the weights for the moment, Proposition 7.3)(a) tells us that there is a unique morphism $F: T \rightarrow \operatorname{End}_{d}^{N}\left[\mathcal{P}^{\prime}\right]$ such that $F_{t}=\left(f_{t}, \gamma_{t}\right)$ for all geometric points $t$ of $T$. To complete the proof, it suffices to show that the assumption that every $F_{t}$ is a model for $\mathcal{P}$ implies that the image $F(T)$ is contained in the subscheme $\operatorname{End}_{d}^{N}[\mathcal{P}]$ of $\operatorname{End}_{d}^{N}\left[\mathcal{P}^{\prime}\right]$. But this is clear, since $F_{t}$ being a model for $\mathcal{P}$ means that

$$
e_{f_{t}}\left(\gamma_{t}(v)\right) \geq \epsilon(v) \quad \text { for all } v \in \mathcal{V}^{\circ}
$$

which is exactly the condition (10) needed to ensure that $\left(f_{t}, \gamma_{t}\right)$ is in the subscheme $\operatorname{End}_{d}^{N}[\mathcal{P}]$ of $\operatorname{End}_{d}^{N}\left[\mathcal{P}^{\prime}\right]$.

(b) We observe that for any $(f, P) \in \operatorname{End}_{d}^{N} \times \mathbb{P}^{N}$ and any $\varphi \in \mathrm{SL}_{N+1}$, we have

$$
e_{\varphi^{-1} \circ f \circ \varphi}\left(\varphi^{-1}(P)\right)=e_{\varphi^{-1} \circ f}(P)=e_{f}(P),
$$

where we use the fact that automorphisms of $\mathbb{P}^{N}$ leave the multiplicity invariant. Since we already know from Proposition 7.3 (b) that $\operatorname{End}_{d}^{N}\left[\mathcal{P}^{\prime}\right]$ is $\mathrm{SL}_{N+1}$-invariant, it follows that the subscheme $\operatorname{End}_{d}^{N}[\mathcal{P}]$ is also $\mathrm{SL}_{N+1}$-invariant. 
Proof of Proposition 7.8. Let $\alpha \in \operatorname{Hom}\left(\mathcal{P}_{1}, \mathcal{P}_{2}\right)$, where we view $\alpha$ as a map $\alpha: \mathcal{V}_{1} \rightarrow \mathcal{V}_{2}$ on vertices. We claim that this gives a morphism

$$
\begin{aligned}
\alpha^{*}: \operatorname{End}_{d}^{N} \times\left(\mathbb{P}^{N}\right)_{\Delta}^{\mathcal{V}_{2}} & \longrightarrow \operatorname{End}_{d}^{N} \times\left(\mathbb{P}^{N}\right)_{\Delta}^{\mathcal{V}_{1}}, \\
(f, P) & \longmapsto(f, P \circ \alpha) .
\end{aligned}
$$

The injectivity of $\alpha$ ensures that $P \circ \alpha: \mathcal{V}_{1} \rightarrow \mathbb{P}^{N}$ avoids the big diagonal.

It remains to check that if $(f, P) \in \operatorname{End}_{d}^{N}\left[\mathcal{P}_{2}\right]$, then $\alpha^{*}(f, P) \in$ $\operatorname{End}_{d}^{N}\left[\mathcal{P}_{1}\right]$. It suffices to check this at the level of geometric points:

$$
\begin{aligned}
& (f, P) \in \operatorname{End}_{d}^{N}\left[\mathcal{P}_{2}\right] \\
& \Longleftrightarrow f \circ P=P \circ \Phi_{2} \text { and } e_{f} \circ P \geq \epsilon_{2} \\
& \text { as maps } \mathcal{V}_{2}^{\circ} \rightarrow \mathbb{P}^{N} \text { and } \mathcal{V}_{2}^{\circ} \rightarrow \mathbb{Z}_{\geq 1} \text {, respectively, } \\
& \Longrightarrow \quad f \circ P \circ \alpha=P \circ \Phi_{2} \circ \alpha \text { and } e_{f} \circ P \circ \alpha \geq \epsilon_{2} \circ \alpha \\
& \text { since } \alpha: \mathcal{V}_{1}^{\circ} \hookrightarrow \mathcal{V}_{2}^{\circ} \text {, } \\
& \Longrightarrow \quad f \circ P \circ \alpha=P \circ \alpha \circ \Phi_{1} \text { and } e_{f} \circ P \circ \alpha \geq \epsilon_{1} \\
& \text { since } \alpha \in \operatorname{Hom}\left(\mathcal{P}_{1}, \mathcal{P}_{2}\right) \text {, } \\
& \Longrightarrow \quad(f, P \circ \alpha) \in \operatorname{End}_{d}^{N}\left[\mathcal{P}_{1}\right] .
\end{aligned}
$$

Hence $\alpha^{*}\left(\operatorname{End}_{d}^{N}\left[\mathcal{P}_{2}\right]\right) \subseteq \operatorname{End}_{d}^{N}\left[\mathcal{P}_{1}\right]$.

\section{The $\mathrm{SL}_{N+1}-\mathrm{Stable}$ Locus of $\operatorname{End}_{d}^{N} \times\left(\mathbb{P}^{N}\right)^{n}$}

In Section 7 we constructed a parameter space $\operatorname{End}_{d}^{N}[\mathcal{P}]$ whose points classify models for $\mathcal{P}$. We would like to take the quotient of this space by the action of $\mathrm{SL}_{N+1}$ so as to construct a moduli space for the isomorphism classes of models for $\mathcal{P}$. In order to obtain a nice quotient, we need to prove that $\operatorname{End}_{d}^{N}[\mathcal{P}]$ is in the stable locus for the action of $\mathrm{SL}_{N+1}$ on $\mathbb{P}^{M} \times\left(\mathbb{P}^{N}\right)^{\mathcal{V}}$ relative to a suitable ample sheaf.

Our primary goal in this section is to prove a simple numerical criterion on the integers $\left(N, d, n, m_{0}, \ldots, n_{n}\right)$ which ensures that $\operatorname{End}_{d}^{N} \times\left(\mathbb{P}^{N}\right)^{n}$ is $\mathrm{SL}_{N+1}$-stable relative to the invertible sheaf $\mathcal{O}\left(m_{0}, \ldots, m_{n}\right)$. This criterion is given in Corollary 8.3, see also Section 11 for a more refined result for $\mathbb{P}^{1}$. Although the corollary is an immediate consequence of the first implication in Theorem 8.1 (b), it requires little extra work to prove Theorem 8.1, and since the more precise statement in the theorem may be of independent interest, we give the full proof.

Theorem 8.1. Fix integers

$$
N \geq 1, \quad d \geq 2, \quad n \geq 0, \quad \boldsymbol{m}:=\left(m_{0}, \ldots, m_{n}\right) \in \mathbb{Z}_{\geq 0}^{n},
$$


and let

$\mathcal{O}(\boldsymbol{m}):=\mathcal{O}_{\mathbb{P}^{M} \times\left(\mathbb{P}^{N}\right)^{n}}\left(m_{0}, m_{1}, \ldots, m_{n}\right) \quad$ and $\quad m_{\Sigma}:=m_{1}+m_{2}+\cdots+m_{n}$.

For each geometric point $\boldsymbol{P}=\left(P_{1}, \ldots, P_{n}\right) \in\left(\mathbb{P}^{N}\right)^{n}$, each linear subspace $L \subseteq \mathbb{P}^{N}$, and each integer $\epsilon \geq 0$, le

$$
\begin{aligned}
C_{\boldsymbol{P}}(L) & :=\sum_{1 \leq \nu \leq n, P_{\nu} \in L} m_{\nu}, \\
D_{\epsilon}(L) & :=\frac{m_{\Sigma}(\operatorname{dim} L+1)+m_{0}(d-1) \operatorname{codim} L}{N+1}+\epsilon .
\end{aligned}
$$

We let $\mathrm{SL}_{N+1}$ act on $\operatorname{End}_{d}^{N} \times\left(\mathbb{P}^{N}\right)^{n}$ as in (3) , i.e., the action on $\operatorname{End}_{d}^{N}$ is via conjugation and on each $\mathbb{P}^{N}$ via the inverse. For a geometric point

$$
(f, \boldsymbol{P}) \in \operatorname{End}_{d}^{N} \times\left(\mathbb{P}^{N}\right)^{n},
$$

we consider the following statements:

(SS) : $\quad(f, \boldsymbol{P})$ is $\mathrm{SL}_{N+1}$-semistable relative to $\mathcal{O}(\boldsymbol{m})$.

$\left(\mathrm{SS}_{\epsilon}\right): \quad C_{\boldsymbol{P}}(L) \leq D_{\epsilon}(L) \quad$ for all linear $L \subsetneq \mathbb{P}^{N}$.

(St): $\quad(f, \boldsymbol{P})$ is $\mathrm{SL}_{N+1}$-stable relative to $\mathcal{O}(\boldsymbol{m})$.

$\left(\mathrm{St}_{\epsilon}\right): \quad C_{\boldsymbol{P}}(L)<D_{\epsilon}(L)$ for all linear $L \subsetneq \mathbb{P}^{N}$.

These statements satisfy the following implications:
(a) $\left(\mathrm{SS}_{0}\right) \Longrightarrow(\mathrm{SS}) \Longrightarrow\left(\mathrm{SS}_{m_{0}}\right)$.

(b) $\left(\mathrm{St}_{0}\right) \Longrightarrow(\mathrm{St}) \Longrightarrow\left(\mathrm{St}_{m_{0}}\right)$.

Remark 8.2. Theorem 8.1 gives necessary and sufficient conditions for a pair $(f, \boldsymbol{P}) \in \operatorname{End}_{d}^{N} \times\left(\mathbb{P}^{N}\right)^{n}$ to be stable relative to $\mathcal{O}(\boldsymbol{m})$. These conditions are stated in terms of the numerical quantities $N, d, n, \boldsymbol{m}$ and the $n$-tuple of points $\boldsymbol{P}$. It would be nice if there were a single condition depending on these quantities that was simultaneously necessary and sufficient, but unfortunately, life is not that simple, and any such condition must also depend on $f$. See Proposition 11.1(c) for an example. We also note that Theorem 8.1 only gives the (semi)stable locus in $\operatorname{End}_{d}^{N} \times\left(\mathbb{P}^{N}\right)^{n}$; it does not describe the full (semi)stable locus in $\mathbb{P}^{M} \times\left(\mathbb{P}^{N}\right)^{n}$.

Before proving Theorem 8.1 , we state a corollary giving a simple numerical criterion for the stability of $\operatorname{End}_{d}^{N} \times\left(\mathbb{P}^{N}\right)^{n}$. See Section 11 for a refined statement for $\mathbb{P}^{1}$.

\footnotetext{
${ }^{4}$ We view $C_{\boldsymbol{P}}(L)$ as a counting function for the points of $\boldsymbol{P}$ that are in $L$, where the points are weighted by $m_{1}, \ldots, m_{n}$.
} 
Corollary 8.3. With notation as in Theorem 8.1, we have:

$$
\begin{aligned}
& m_{0} \geq \frac{m_{\Sigma}}{d-1} \Longrightarrow \operatorname{End}_{d}^{N} \times\left(\mathbb{P}^{N}\right)^{n} \text { is } \mathrm{SL}_{N+1} \text {-semistable for } \mathcal{O}(\boldsymbol{m}) . \\
& m_{0}>\frac{m_{\Sigma}}{d-1} \Longrightarrow \operatorname{End}_{d}^{N} \times\left(\mathbb{P}^{N}\right)^{n} \text { is } \mathrm{SL}_{N+1} \text {-stable for } \mathcal{O}(\boldsymbol{m}) .
\end{aligned}
$$

We note that the $n=0$ case of Theorem 8.1 is the classical result that $\operatorname{End}_{d}^{N}$ is $\mathrm{SL}_{N+1}$-stable relative to $\mathcal{O}(1)$; cf. Theorem 5.1. It is also instructive to note that the $\boldsymbol{m}=(0,1, \ldots, 1)$ case of Theorem 8.1 gives the following classical result on stability of points in $\left(\mathbb{P}^{N}\right)^{n}$. Thus our Theorem 8.1 is, in some sense, an amalgamation of Theorems 5.1 and 8.4, although it is not a direct consequence of these two results.

Theorem 8.4 (Mumford). Let $n \geq 1$ and $N \geq 1$. A geometric point $\boldsymbol{P}=\left(P_{1}, \ldots, P_{n}\right) \in\left(\mathbb{P}^{N}\right)^{n}$ is $\mathrm{SL}_{N+1}$-stable relative to $\mathcal{O}(1,1, \ldots, 1)$ if and only if 5

$$
\#\left\{\nu: P_{\nu} \in L\right\}<\frac{n(\operatorname{dim} L+1)}{N+1} \quad \text { for all linear } L \subsetneq \mathbb{P}^{N} .
$$

Proof. Mumford proves this directly from the definition of stability in [50, Chapter 3]; see Definition 3.7/Proposition 3.4 and Theorem 3.8. A proof via the numerical criterion may be found in [12, Example 3.3.21].

Proof of Theorem 8.1. We note that every $(f, \boldsymbol{P}) \in \operatorname{End}_{d}^{N} \times\left(\mathbb{P}^{N}\right)^{n}$ has finite stabilizer in $\mathrm{SL}_{N+1}$, since Theorem 5.1(b) says that $f$ itself has finite stabilizer.

In order to use the numerical criterion (Theorem 4.3) to study stability and semistability, we choose an arbitrary non-trivial 1-parameter subgroup $\ell: \mathbb{G}_{m} \hookrightarrow \mathrm{SL}_{N+1}(k)$, and we choose coordinates on $\mathbb{P}^{N}$ to diagonalize the action as follows:

$$
\ell(\alpha):=\left(\begin{array}{cccc}
\alpha^{k_{0}} & & & \\
& \alpha^{k_{1}} & & \\
& & \ddots & \\
& & & \alpha^{k_{N}}
\end{array}\right) \in \mathrm{SL}_{N+1}
$$

with

$$
k_{0} \leq k_{1} \leq \cdots \leq k_{N}, \quad k_{0}+k_{1}+\cdots+k_{N}=0, \quad \text { and } \quad k_{N}>0 .
$$

We write endomorphisms $f \in \operatorname{End}_{d}^{N}$ using the notation (2) described in Section 5. An $\ell$-diagonalized basis for the global sections of $\mathcal{O}(1)$

\footnotetext{
${ }^{5}$ One can show that (20) implies that $\boldsymbol{P}$ has trivial stabilizer; see [50, Proposition 3.6].
} 
on $\operatorname{End}_{d}^{N}$ is given by

$$
\left\{a_{\rho}(\boldsymbol{e}): 0 \leq \rho \leq N \text { and }|\boldsymbol{e}|=d\right\} .
$$

The action of the 1-parameter subgroup (21) on this basis is

$$
\ell(\alpha) \star a_{\rho}(\boldsymbol{e})=\alpha^{k_{0} e_{0}+\cdots+k_{N} e_{N}-k_{\rho}} a_{\rho}(\boldsymbol{e}) .
$$

For later use, we define

$$
\boldsymbol{d}:=(d, 0,0, \ldots, 0),
$$

so $a_{\rho}(\boldsymbol{d})$ is the coefficient of $x_{0}^{d}$ in $f_{\rho}$. The assumption that $f \in \operatorname{End}_{d}^{N}$ implies that there exists some $\rho^{\prime}$ with

$$
a_{\rho^{\prime}}(\boldsymbol{d}) \neq 0,
$$

since otherwise $f_{0}, \ldots, f_{N}$ would all vanish at $[1,0, \ldots, 0]$, say.

Next we turn to $\left(\mathbb{P}^{N}\right)^{n}$. We let $[U(0), \ldots, U(N)]$ be homogeneous coordinates on $\mathbb{P}^{N}$, so each $U(i)$ is a section of $\mathcal{O}_{\mathbb{P}^{N}}(1)$, and the action of $\ell$ on these sections is via

$$
\ell(\alpha) \star U(i)=\alpha^{-k_{i}} U(i) .
$$

(The negative exponent is due to the fact that $\ell$ acts on points via the inverse matrix.)

More generally, for $1 \leq \nu \leq n$, we let

$$
\left[U_{\nu}(0), \ldots, U_{\nu}(N)\right]=\left\{\begin{array}{l}
\text { homogeneous coordinates on } \\
\text { the } \nu \text { th copy of } \mathbb{P}^{N} \text { in }\left(\mathbb{P}^{N}\right)^{n}
\end{array}\right\}
$$

(Formally, we have $U_{\nu}(i):=U(i) \circ \operatorname{proj}_{\nu}$, where $\operatorname{proj}_{\nu}:\left(\mathbb{P}^{N}\right)^{n} \rightarrow \mathbb{P}^{N}$ is projection on the $\nu$ th factor.)

For our given point $\boldsymbol{P}=\left(P_{1}, \ldots, P_{n}\right) \in\left(\mathbb{P}^{N}\right)^{n}$, we let

$$
\begin{aligned}
j_{\nu} & :=\text { largest } j \text { so that the } j \text { th coordinate of } P_{\nu} \text { is non-zero } \\
& :=\text { the index with } U_{\nu}\left(j_{\nu}\right) \neq 0 \text { and } U_{\nu}(j)=0 \text { for } j>j_{\nu} .
\end{aligned}
$$

Then with these definitions, we have

$$
U_{\nu}\left(j_{\nu}\right) \neq 0 \text { for all } 1 \leq \nu \leq n .
$$

We now consider a general $\ell$-diagonalized global section of $\mathcal{O}(\boldsymbol{m})$. Such a section is a product of $m_{0}$ copies of sections of the form $a_{\rho}(\boldsymbol{e})$ multiplied by $m_{1}$ copies of sections of the form $U_{1}(i)$, multiplied by $m_{2}$ copies of sections of the form $U_{2}(i)$, etc. Thus such a section has the form

$$
s:=\prod_{\mu=1}^{m_{0}} a_{\rho_{\mu}}\left(\boldsymbol{e}_{\mu}\right) \cdot \prod_{\nu=1}^{n} \prod_{\lambda=1}^{m_{\nu}} U_{\nu}\left(i_{\nu, \lambda}\right) .
$$


The section $s$ depends on the choice of the quantities

$$
\rho_{\mu}, \quad \boldsymbol{e}_{\mu}=\left(e_{\mu, 0}, \ldots, e_{\mu, N}\right), \quad i_{\nu, \lambda},
$$

but for convenience we omit this dependence from the notation and always take $s$ to be defined by (26).

The action of $\ell$ on $s$ is given by

$$
\ell(\alpha) \star s=\alpha^{E(s)} s \text { with } E(s):=\sum_{\mu=1}^{m_{0}}\left(\sum_{\sigma=0}^{N} k_{\sigma} e_{\mu, \sigma}-k_{\rho_{\mu}}\right)-\sum_{\nu=1}^{n} \sum_{\lambda=1}^{m_{\nu}} k_{i_{\nu, \lambda}} .
$$

For $-1 \leq r \leq N$, we define an increasing sequence of nested linear subspaces

$$
L_{r}:=\{U(r+1)=\cdots=U(N)=0\} \subset \mathbb{P}^{N},
$$

so in particular $L_{-1}=\emptyset$ and $L_{N}=\mathbb{P}^{N}$. We have

$$
\operatorname{dim} L_{r}=r \quad \text { for all } 0 \leq r \leq N .
$$

We note that the definition of $j_{\nu}$ says exactly that

$$
P_{\nu} \in L_{j_{\nu}} \backslash L_{j_{\nu}-1}
$$

This criterion plays the key role in relating the numerical invariant to membership of the $P_{\nu}$ in linear subspaces.

Using the notation $C_{\boldsymbol{P}}(L)$ and $D_{\epsilon}(L)$ defined by (18) and (19), our next step is to prove the following two formulas, which will be needed later:

$$
\begin{aligned}
\sum_{r=0}^{N} C_{\boldsymbol{P}}\left(L_{r}\right)\left(k_{r}-k_{r+1}\right) & =\sum_{\nu=1}^{n} m_{\nu} k_{j_{\nu}} \\
\sum_{r=0}^{N} D_{0}\left(L_{r}\right)\left(k_{r}-k_{r+1}\right) & =m_{0}(d-1) k_{0}
\end{aligned}
$$

where for convenience we set $k_{N+1}=0$.

In order to prove (31), we compute

$$
\begin{aligned}
\sum_{\nu=1}^{n} m_{\nu} k_{j_{\nu}} & =\sum_{r=0}^{N}\left(\sum_{\substack{1 \leq \nu \leq n \\
j_{\nu}=r}} m_{\nu}\right) k_{r} & & \text { grouping } j_{\nu} \text { by value } \\
& =\sum_{r=0}^{N}\left(\sum_{\substack{1 \leq \nu \leq n \\
P_{\nu} \in L_{r} \backslash L_{r-1}}} m_{\nu}\right) k_{r} & & \text { from (130) }
\end{aligned}
$$




$$
\begin{aligned}
& =\sum_{r=0}^{N} C_{\boldsymbol{P}}\left(L_{r}\right) k_{r}-\sum_{r=0}^{N} C_{\boldsymbol{P}}\left(L_{r-1}\right) k_{r} \quad \text { definition of } C_{\boldsymbol{P}}(L), \\
& =\sum_{r=0}^{N} C_{\boldsymbol{P}}\left(L_{r}\right) k_{r}-\sum_{r=0}^{N} C_{\boldsymbol{P}}\left(L_{r}\right) k_{r+1} \quad \text { using } L_{-1}=\emptyset, k_{N+1}=0, \\
& =\sum_{r=0}^{N} C_{\boldsymbol{P}}\left(L_{r}\right)\left(k_{r}-k_{r+1}\right) .
\end{aligned}
$$

This completes the proof of (31).

We now turn to the proof of (32). To ease notation, we let

$$
A=\frac{m_{\Sigma}-m_{0}(d-1)}{N+1} \quad \text { and } \quad B=\frac{m_{\Sigma}+m_{0}(d-1) N}{N+1} .
$$

Using $\operatorname{dim} L_{r}=r$ and $\operatorname{codim} L_{r}=N-r$, we see that from (19) that

$$
D_{0}\left(L_{r}\right)=\frac{m_{\Sigma}(r+1)+m_{0}(d-1)(N-r)}{N+1}=A r+B
$$

Then

$$
\begin{aligned}
\sum_{r=0}^{N} D_{0}\left(L_{r}\right)\left(k_{r}-k_{r+1}\right) & \left.=\sum_{r=0}^{N}(A r+B)\left(k_{r}-k_{r+1}\right) \quad \text { from (33) }\right) \\
& =\sum_{r=0}^{N} A r k_{r}-\sum_{r=0}^{N} A r k_{r+1}+B k_{0} \\
& =\sum_{r=0}^{N} A r k_{r}-\sum_{r=1}^{N+1} A(r-1) k_{r}+B k_{0} \\
& =A \sum_{r=1}^{N} k_{r}+B k_{0} \quad \text { since } k_{N+1}=0, \\
& =(-A+B) k_{0} \quad \text { since } \sum_{r=0}^{N} k_{r}=0 \text { from (22) }, \\
& =m_{0}(d-1) k_{0} \quad \text { since } B-A=m_{0}(d-1) .
\end{aligned}
$$

This completes the proof of (32).

We now resume the proof of Theorem 8.1, so we consider the product End $_{d}^{N} \times\left(\mathbb{P}^{N}\right)^{n}$ and the $\ell$-diagonalized global sections $s$ for the invertible sheaf $\mathcal{O}(\boldsymbol{m})=\mathcal{O}\left(m_{0}, m_{1}, \ldots, m_{n}\right)$ as defined by (26) $)$. 
A key observation is that we can specify parameters for $s$ so that $s$ does not vanish at our given point $(f, \boldsymbol{P})$ :

$$
s \neq 0 \text { at }(f, \boldsymbol{P}) \text { if }\left\{\begin{array}{cl}
\boldsymbol{e}_{\mu}=\boldsymbol{d} & \text { for all } \mu, \\
\rho_{\mu}=\rho^{\prime} & \text { for all } \mu, \text { and } \\
i_{\nu, \lambda}=j_{\nu} & \text { for all } \nu \text { and all } \lambda,
\end{array}\right.
$$

where $\boldsymbol{d}$ and $\rho^{\prime}$ are defined by (23) and $j_{\nu}$ is defined by (24).

Proof of $\left(\mathrm{SS}_{0}\right) \Longrightarrow(\mathrm{SS})$ and $\left(\mathrm{St}_{0}\right) \Longrightarrow(\mathrm{St})$

The numerical criterion (Theorem 4.3) says that $(f, \boldsymbol{P})$ is semistable relative to $\mathcal{O}(\boldsymbol{m})$ if and only if $\mu^{\mathcal{O}(\boldsymbol{m})}((f, \boldsymbol{P}), \ell) \geq 0$ for every 1parameter subgroup $\ell: \mathbb{G}_{m} \rightarrow \mathrm{SL}_{N+1}$, and similarly for stable with a strict inequality for $\mu$. Without loss of generality, we choose coordinates so that $\ell$ is diagonalized and has the form given by (21) and (22), and then we write $f$ and $\boldsymbol{P}$ using the $\ell$-diagonalized global sections $s$ of $\mathcal{O}(\boldsymbol{m})$ described in (26). Having done this, and recalling from (27) the notation $\ell \star s=\alpha^{E(s)} s$, we estimate the numerical invariant as follows:

$$
\begin{aligned}
\mu^{\mathcal{O}(\boldsymbol{m})} & ((f, \boldsymbol{P}), \ell) \\
& =\max \{-E(s): s \text { is defined by (26) }) \text { and } s(f, \boldsymbol{P}) \neq 0\} \\
& =\max _{s(f, \boldsymbol{P}) \neq 0}-\left(\sum_{\mu=1}^{m_{0}}\left(\sum_{\sigma=0}^{N} k_{\sigma} e_{\mu, \sigma}-k_{\rho_{\mu}}\right)-\sum_{\nu=1}^{n} \sum_{\lambda=1}^{m_{\nu}} k_{i_{\nu, \lambda}}\right) \text { from (27), } \\
& \geq \sum_{\mu=1}^{m_{0}}\left(-k_{0} d+k_{\rho^{\prime}}\right)+\sum_{\nu=1}^{n} \sum_{\lambda=1}^{m_{\nu}} k_{j_{\nu}} \quad \text { using the } s \text { from (34), } \\
& \geq-m_{0} k_{0}(d-1)+\sum_{\nu=1}^{n} m_{\nu} k_{j_{\nu}} \quad \text { since } k_{0}=\min k_{\rho} \text { from (22) }, \\
& =-\sum_{r=0}^{N} D_{0}\left(L_{r}\right)\left(k_{r}-k_{r+1}\right)+\sum_{r=0}^{N} C_{\boldsymbol{P}}\left(L_{r}\right)\left(k_{r}-k_{r+1}\right) \\
& =\sum_{r=0}^{N}\left(D_{0}\left(L_{r}\right)-C_{\boldsymbol{P}}\left(L_{r}\right)\right)\left(k_{r+1}-k_{r}\right) .
\end{aligned}
$$

We next use the fact that $L_{N}=\mathbb{P}^{N}$ to observe that

$$
D_{0}\left(L_{N}\right)=C_{\boldsymbol{P}}\left(L_{N}\right)=m_{\Sigma}
$$


so the $r=N$ term in the sum vanishes. Hence

$$
\mu^{\mathcal{O}(\boldsymbol{m})}((f, \boldsymbol{P}), \ell) \geq \sum_{r=0}^{N-1}\left(D_{0}\left(L_{r}\right)-C_{\boldsymbol{P}}\left(L_{r}\right)\right)(\underbrace{k_{r+1}-k_{r}}_{\geq 0 \text { from }[22)}) .
$$

We stress that eliminating the $r=N$ term is crucial, since the inequality $k_{r+1}-k_{r} \geq 0$ is not true for $r=N$.

Suppose now that $\left(\mathrm{SS}_{0}\right)$ holds. Applying $\left(\mathrm{SS}_{0}\right)$ with $L=L_{r}$ tells us that $C_{\boldsymbol{P}}\left(L_{r}\right) \leq D_{0}\left(L_{r}\right)$ for all $0 \leq r<N$, while as noted earlier, we have $k_{r+1} \geq k_{r}$ for all $0 \leq r<N$. (Again, note that this is not true for $r=N$.) Thus the lower bound for $\mu^{\mathcal{O}(\boldsymbol{m})}((f, \boldsymbol{P}), \ell)$ given by (35) is a sum of non-negative terms, and hence $\mu^{\mathcal{O}(\boldsymbol{m})}((f, \boldsymbol{P}), \ell) \geq 0$. The numerical criterion (Theorem 4.3) then tells us that $(f, \boldsymbol{P})$ is a semistable point, i.e., (SS) is true.

We turn now to stability and assume that $\left(\mathrm{St}_{0}\right)$ holds. Applying $\left(\mathrm{St}_{0}\right)$ with $L=L_{r}$ gives the strict inequality $C_{\boldsymbol{P}}\left(L_{r}\right)<D_{0}\left(L_{r}\right)$, so the lower bound for $\mu^{\mathcal{O}(\boldsymbol{m})}((f, \boldsymbol{P}), \ell)$ in (35) is strictly positive unless

$$
k_{r+1}=k_{r} \quad \text { for all } 0 \leq r<N .
$$

But this would imply that $k_{0}=k_{1}=\cdots=k_{N}$, and then the fact (22) that $\sum_{r=0}^{N} k_{r}=0$ would force $k_{0}=\cdots=k_{N}=0$, contradicting the assumption that $\ell$ is a non-trivial 1-parameter subgroup. Hence the lower bound is strictly positive, i.e., $\mu^{\mathcal{O}(\boldsymbol{m})}((f, \boldsymbol{P}), \ell)>0$, and the numerical criterion then tells us that $(f, \boldsymbol{P})$ is a stable point. Hence (St) is true.

$$
\text { Proof of }(\mathrm{SS}) \Longrightarrow\left(\mathrm{SS}_{m_{0}}\right) \text { and }(\mathrm{St}) \Longrightarrow\left(\mathrm{St}_{m_{0}}\right)
$$

Let $(f, \boldsymbol{P}) \in \operatorname{End}_{d}^{N} \times\left(\mathbb{P}^{N}\right)^{n}$ and let $L \subset \mathbb{P}^{N}$. We claim that there exists a non-trivial 1-parameter subgroup $\ell: \mathbb{G}_{m} \hookrightarrow \mathrm{SL}_{N+1}$, depending on $(N, d, n, \boldsymbol{m}, \boldsymbol{P})$, such that

$$
\mu^{\mathcal{O}(\boldsymbol{m})}((f, \boldsymbol{P}), \ell) \leq\left(D_{m_{0}}(L)-C_{\boldsymbol{P}}(L)\right)(N+1) .
$$

To prove this claim, we let

$$
r:=\operatorname{dim} L \quad \text { and } \quad c:=\#\left\{\nu: P_{\nu} \in L\right\} .
$$

Changing coordinates, we may assume without loss of generality that

$$
L=\{U(r+1)=\cdots=U(N)=0\} \subset \mathbb{P}^{N} .
$$

By definition, exactly $c$ of the $P_{\nu}$ are in $L$, so relabeling the $P_{\nu}$ (hence also the $m_{\nu}$ ), we may assume that

$$
P_{1}, P_{2}, \ldots, P_{c} \in L
$$

In other words, we have

$$
U_{\nu}(i)=0 \text { for all } 1 \leq \nu \leq c \text { and all } i \geq r+1 .
$$


We take for $\ell$ the following 1-parameter subgroup:



It follows from (37) and our choice of $\ell$ that if the section $s$ defined by (26) is non-zero at $(f, \boldsymbol{P})$, then in particular we must have

$$
\begin{aligned}
\sum_{\nu=1}^{n} \sum_{\lambda=1}^{m_{\nu}} k_{i_{\nu, \lambda}} & =\sum_{\nu=1}^{c} \sum_{\lambda=1}^{m_{\nu}} \underbrace{k_{i_{\nu, \lambda}}}_{i_{\nu, \lambda} \leq r \text { from }}+\sum_{\nu=c+1}^{n} \sum_{\lambda=1}^{m_{\nu}} k_{i_{\nu, \lambda}} \\
& \leq \sum_{\nu=1}^{c} \sum_{\lambda=1}^{m_{\nu}}-(N-r)+\sum_{\nu=c+1}^{n} \sum_{\lambda=1}^{m_{\nu}}(r+1) \\
& =-(N-r) \sum_{\nu=1}^{c} m_{\nu}+\sum_{\nu=c+1}^{n} m_{\nu}(r+1) \\
& =-(N-r) C_{\boldsymbol{P}}(L)+\left(\sum_{\nu=1}^{n} m_{\nu}-C_{\boldsymbol{P}}(L)\right)(r+1) \\
& =-(N-r) C_{\boldsymbol{P}}(L)+\left(m_{\Sigma}-C_{\boldsymbol{P}}(L)\right)(r+1) \\
& =-C_{\boldsymbol{P}}(L)(N+1)+m_{\Sigma}(r+1) .
\end{aligned}
$$

We combine this with the trivial bounds

$$
\begin{aligned}
-\sum_{\mu=1}^{m_{0}} \sum_{\sigma=0}^{N} k_{\sigma} e_{\mu, \sigma} & \leq \sum_{\mu=1}^{m_{0}} \sum_{\sigma=0}^{N}(N-r) e_{\mu, \sigma}=m_{0}(N-r) d \\
\sum_{\mu=1}^{m_{0}} k_{\rho_{\mu}} & \leq m_{0}(r+1),
\end{aligned}
$$

to estimate

$$
\begin{aligned}
\mu^{\mathcal{O}(\boldsymbol{m})}( & (f, \boldsymbol{P}), \ell) \\
& =\max \{-E(s): s \text { is defined by (26) and } s(f, \boldsymbol{P}) \neq 0\} \\
& =\max _{s(f, \boldsymbol{P}) \neq 0}-\sum_{\mu=1}^{m_{0}} \sum_{\sigma=0}^{N} k_{\sigma} e_{\mu, \sigma}+\sum_{\mu=1}^{m_{0}} k_{\rho_{\mu}}+\sum_{\nu=1}^{n} \sum_{\lambda=1}^{m_{\nu}} k_{i_{\nu, \lambda}} \quad \text { from (27) }
\end{aligned}
$$




$$
\begin{gathered}
\leq m_{0}(N-r) d+m_{0}(r+1)-C_{\boldsymbol{P}}(L)(N+1)+m_{\Sigma}(r+1) \\
\text { from (38), (139), and (40), } \\
=\left(D_{m_{0}}(L)-C_{\boldsymbol{P}}(L)\right)(N+1) \quad \text { by definition of } D_{\epsilon}(L) .
\end{gathered}
$$

This completes the proof of (36).

We are going to prove contrapositives. So we assume first that $\left(\mathrm{SS}_{m_{0}}\right)$ is false, which means that there is a subspace $L \subsetneq \mathbb{P}^{N}$ such that $C_{\boldsymbol{P}}(L)>D_{m_{0}}(L)$. It follows from (36) that there is an $\ell$ satisfying $\mu^{\mathcal{O}(\boldsymbol{m})}((f, \boldsymbol{P}), \ell)<0$. The numerical criterion (Theorem 4.3) then implies that $(f, \boldsymbol{P})$ is not semistable, i.e., (SS) is false.

Similarly, if we assume that that $\left(\mathrm{St}_{m_{0}}\right)$ is false, then there is a subspace $L \subsetneq \mathbb{P}^{N}$ such that $C_{\boldsymbol{P}}(L) \geq D_{m_{0}}(L)$, and (36) gives an $\ell$ with $\mu^{\mathcal{O}(\boldsymbol{m})}((f, \boldsymbol{P}), \ell) \leq 0$. So the numerical criterion implies that $(f, \boldsymbol{P})$ is not stable, i.e., (St) is false.

Proof of Corollary 8.3. We always have the trivial inequality

$$
C_{\boldsymbol{P}}(L) \leq m_{\Sigma}
$$

where the upper bound comes from the case that $L$ contains every point in $\boldsymbol{P}$. So condition $\left(\mathrm{SS}_{0}\right)$ is automatically satisfied provided

$$
m_{\Sigma} \leq \frac{m_{\Sigma}(r+1)+m_{0}(d-1)(N-r)}{N+1} \text { for all } 0 \leq r<N,
$$

and similarly $\left(\mathrm{St}_{0}\right)$ is true if (41) holds with a strict inequality. Since

$$
\frac{m_{\Sigma}(r+1)+m_{0}(d-1)(N-r)}{N+1}-m_{\Sigma}=\frac{\left(m_{0}(d-1)-m_{\Sigma}\right)(N-r)}{N+1},
$$

so we see that $m_{0}(d-1) \geq m_{\Sigma}$ ensures the required non-negativity, and $m_{0}(d-1)>m_{\Sigma}$ ensures positivity.

\section{Formal Models and Stability of $\operatorname{End}_{d}^{N}[\mathcal{P}]$}

In this section we describe the portrait parameter space $\operatorname{End}_{d}^{N}[\mathcal{P}]$ as a finite cover of the parameter space associated to its subportrait of endpoints. In particular, if $\mathcal{P}$ is preperiodic, i.e., has no endpoints, then we obtain a description of the natural projection map $\operatorname{End}_{d}^{N}[\mathcal{P}] \rightarrow$ $\operatorname{End}_{d}^{N}$. If the endpoint portrait parameter space is $\mathrm{SL}_{N+1}$-stable, we use standard GIT arguments to show that $\operatorname{End}_{d}^{N}[\mathcal{P}]$ is also $\mathrm{SL}_{N+1}$-stable.

Theorem 9.1. Let $N \geq 1$, let $d \geq 2$, let $\mathcal{P}=\left(\mathcal{V}^{\circ}, \mathcal{V}, \Phi, \epsilon\right)$ be a portrait such that the scheme

$$
\operatorname{End}_{d}^{N}[\mathcal{P}] \subset \mathbb{P}^{M} \times\left(\mathbb{P}^{N}\right)^{\mathcal{V}}
$$


constructed in Theorem 7.7 is non-empty 6 let

$$
\overline{\operatorname{End}}_{d}^{N}[\mathcal{P}]:=\text { Zariski closure of } \operatorname{End}_{d}^{N}[\mathcal{P}] \text { in } \operatorname{End}_{d}^{N} \times\left(\mathbb{P}^{N}\right)^{\mathcal{V}}
$$

and let

$$
\Pi: \mathbb{P}^{M} \times\left(\mathbb{P}^{N}\right)^{\mathcal{V}} \longrightarrow \mathbb{P}^{M} \times\left(\mathbb{P}^{N}\right)^{\mathcal{V} \backslash \mathcal{V}^{\circ}}
$$

be the projection map that discards the copies of $\mathbb{P}^{N}$ associated to the vertices in $\mathcal{V}^{\circ}$, i.e., $\Pi$ discards the vertices of $\mathcal{P}$ at which $\Phi$ is defined.

(a) The map

$$
\Pi_{\mathcal{P}}: \overline{\operatorname{End}}_{d}^{N}[\mathcal{P}] \longrightarrow \operatorname{End}_{d}^{N} \times\left(\mathbb{P}^{N}\right)^{\mathcal{V} \backslash \mathcal{V}^{\circ}}
$$

induced by $\Pi$ is a finite morphism.

(b) Both $\overline{\operatorname{End}}_{d}^{N}[\mathcal{P}]$ and $\operatorname{End}_{d}^{N}[\mathcal{P}] \times\left(\mathbb{P}^{N}\right)^{\mathcal{V} \backslash \mathcal{V}^{\circ}}$ are $\mathrm{SL}_{N+1}$-invariant subschemes of $\mathbb{P}^{M} \times\left(\mathbb{P}^{N}\right)^{\mathcal{V}}$.

(c) Let $m$ be an integer satisfying 8

$$
m>\frac{1}{d-1} \#\left(\mathcal{V} \backslash \mathcal{V}^{\circ}\right)
$$

Then the schemes $\overline{\operatorname{End}}_{d}^{N}[\mathcal{P}]$ and $\operatorname{End}_{d}^{N}[\mathcal{P}]$ are contained in the $\mathrm{SL}_{N+1}$-stable locus of $\mathbb{P}^{M} \times\left(\mathbb{P}^{N}\right)^{\mathcal{V}}$ relative to the invertible sheaf

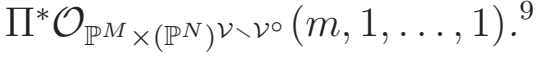

Definition 9.2. Let $\overline{\operatorname{End}}_{d}^{N}[\mathcal{P}]$ be as in Theorem 9.1. A point

$$
\left(f,\left(P_{v}\right)_{v \in \mathcal{V}}\right) \in \overline{\operatorname{End}}_{d}^{N}[\mathcal{P}](k)
$$

is called a formal model for the portrait $\mathcal{P}$ over the field $k$. Note that if we view $P$ as a map $P: \mathcal{V} \rightarrow \mathbb{P}^{N}(k)$, then $(f, P)$ is a model for $\mathcal{P}$ as per Definition 6.3 if and only if $P$ is injective. However, there may be points in $\overline{\operatorname{End}}_{d}^{N}[\mathcal{P}](k)$ for which this injectivity is not true. On the other hand, for any given $f$, there may or may not be a non-injective map $P: \mathcal{V} \rightarrow \mathbb{P}^{N}(k)$ for which $(f, P)$ is in $\overline{\operatorname{End}}_{d}^{N}[\mathcal{P}]$. This is a familiar phenomenon in dynamics, even for polynomials in $\mathbb{C}[z]$, where a point may have formal period strictly larger than its actual period; cf. 58,

\footnotetext{
${ }^{6}$ See Theorems 14.2 and 15.7 and Proposition 15.1 for various necessary and sufficient conditions on $\mathcal{P}$ so that $\operatorname{End}_{d}^{N}[\mathcal{P}] \neq \emptyset$, and formulas for $\operatorname{dim} \operatorname{End}_{d}^{N}[\mathcal{P}]$.

${ }^{7}$ But note that if we replace $\overline{\operatorname{End}}_{d}^{N}[\mathcal{P}]$ with $\operatorname{End}_{d}^{N}[\mathcal{P}]$, then the map need not be proper; see Example 9.4 .

${ }^{8}$ In particular, if $\mathcal{P}$ is a preperiodic portrait, it suffices to take $m=1$. One only needs larger values of $m$ if $\mathcal{P}$ has endpoints, or in graph-speak, if the directed graph $\mathcal{P}$ has sinks.

${ }^{9}$ More generally, if we let $n=\#\left(\mathcal{V} \backslash \mathcal{V}^{\circ}\right)$ and we replace (42) with $m_{0}>\left(m_{1}+\right.$ $\left.\cdots+m_{n}\right) /(d-1)$, then the indicated schemes are in the stable locus relative to $\Pi^{*} \mathcal{O}\left(m_{0}, m_{1}, \ldots, m_{n}\right)$, and similarly in Theorem 10.1
} 
Section 4.1]. The terminology "formal period" for rational maps on $\mathbb{P}^{1}$ is due originally to Milnor.

Proof of Theorem 9.1. (a) We first treat the case that the portrait $\mathcal{P}$ is unweighted, i.e., we assume that $\epsilon(v)=1$ for all $v \in \mathcal{V}^{\circ}$. We modify the definition of $\operatorname{End}_{d}^{N}[\mathcal{P}]$ as described by (17) and (8) to create a somewhat larger subscheme by putting the big diagonal back into $\left(\mathbb{P}^{N}\right)_{\Delta}^{\mathcal{V}}$. Explicitly, we let

$$
\bar{\Psi}_{v, v^{\prime}}: \operatorname{End}_{d}^{N} \times\left(\mathbb{P}^{N}\right)^{\mathcal{V}} \rightarrow \mathbb{P}^{N} \times \mathbb{P}^{N}, \quad \bar{\Psi}_{v, v^{\prime}}=\left(\pi_{v} \times \pi_{v^{\prime}}\right) \circ F,
$$

where note that the domain now uses $\left(\mathbb{P}^{N}\right)^{\mathcal{V}}$ instead of $\left(\mathbb{P}^{N}\right)_{\Delta}^{\mathcal{V}}$. We then define a scheme $W$ by

$$
W:=\bigcap_{v \in \mathcal{V}^{\circ}} \bar{\Psi}_{v, \Phi(v)}^{-1}(\Delta) \subset \operatorname{End}_{d}^{N} \times\left(\mathbb{P}^{N}\right)^{\mathcal{V}} .
$$

We start with three observations about $W$.

(1) $W$ is a closed subscheme of $\operatorname{End}_{d}^{N} \times\left(\mathbb{P}^{N}\right)^{\mathcal{V}}$. Proof: Since $\Delta$ is a closed subscheme of $\mathbb{P}^{N} \times \mathbb{P}^{N}$, and since $\bar{\Psi}_{v, w}$ is a morphism, each $\bar{\Psi}_{v, w}^{-1}(\Delta)$ is a closed subscheme of $\operatorname{End}_{d}^{N} \times\left(\mathbb{P}^{N}\right)^{\mathcal{V}}$. Thus $W$ is a finite intersection of closed subschemes, hence $W$ is a closed subscheme.

(2) $\operatorname{End}_{d}^{N}[\mathcal{P}]$ is contained in $W$. Proof: From the definitions of $\Psi$ and $\bar{\Psi}$ we see that $\Psi_{v, w}^{-1}(\Delta) \subseteq \bar{\Psi}_{v, w}^{-1}(\Delta)$, and then $\operatorname{End}_{d}^{N}[\mathcal{P}] \subset W$ follows by taking an intersection of these inclusions.

(3) $\overline{\operatorname{End}}_{d}^{N}[\mathcal{P}]$ is equal to the closure of $\operatorname{End}_{d}^{N}[\mathcal{P}]$ in $W$. Proof: By definition, $\overline{\operatorname{End}}_{d}^{N}[\mathcal{P}]$ is the closure of $\operatorname{End}_{d}^{N}[\mathcal{P}]$ in $\operatorname{End}_{d}^{N} \times\left(\mathbb{P}^{N}\right)^{\mathcal{V}}$. From (2), it follows that $\overline{\operatorname{End}}_{d}^{N}[\mathcal{P}]$ is the closure of $\operatorname{End}_{d}^{N}[\mathcal{P}]$ in the closure of $W$, but from (1), the closure of $W$ is just $W 10$

To ease notation, we let

$$
\mathcal{Z}=\mathcal{V} \backslash \mathcal{V}^{\circ}
$$

We define

$$
\Pi_{W}: W \longrightarrow \operatorname{End}_{d}^{N} \times\left(\mathbb{P}^{N}\right)^{\mathcal{Z}}
$$

to be the restriction of $\Pi$ to $W$, so in particular $\Pi_{\mathcal{P}}$ is the restriction of $\Pi_{W}$ to $\overline{\operatorname{End}}_{d}^{N}[\mathcal{P}]$. Let $k$ be an algebraically closed field, and consider a geometric point

$$
\left(f,\left(P_{v}\right)_{v \in \mathcal{V}}\right) \in W(k) .
$$

By construction we have

$$
f\left(P_{v}\right)=P_{\Phi(v)} \quad \text { for all } v \in \mathcal{V}^{\circ},
$$

since the only change between the definition (8) of $\operatorname{End}_{d}^{N}[\mathcal{P}]$ and the definition (43) of $W$ is that we have dropped the requirement that the

\footnotetext{
${ }^{10}$ In general, $W$ may be significantly larger than $\overline{\operatorname{End}}_{d}^{N}[\mathcal{P}]$; see Example 9.5
} 
points $P_{v}$ be distinct. The use of the inverse image $\bar{\Psi}_{v, \Phi(v)}^{-1}(\Delta)$ in (43) still forces the geometric points of $W$ to satisfy (45).

We start by verifying that the map $\Pi_{W}$ is quasi-finite, i.e., has finite fibers at all geometric points. Fix a geometric point

$$
\left(f,\left(Q_{z}\right)_{z \in \mathcal{Z}}\right) \in\left(\operatorname{End}_{d}^{N} \times\left(\mathbb{P}^{N}\right)^{\mathcal{Z}}\right)(k),
$$

and consider the points

$$
\left(f,\left(P_{v}\right)_{v \in \mathcal{V}}\right) \in \Pi_{W}^{-1}\left(f,\left(Q_{z}\right)_{z \in \mathcal{Z}}\right) .
$$

This means that $P_{z}=Q_{z}$ for all $z \in \mathcal{Z}$, and we need to show that for each $v \in \mathcal{V}^{\circ}$, there are only finitely many possibilities for $P_{v}$. We consider two cases.

First, if $v \in \mathcal{V}^{\circ}$ is preperiodic for $\Phi$, then (45) implies that $P_{v}$ is preperiodic for $f$, and the total length of the orbit is clearly bounded by $\# \mathcal{V}$. We now use the fact that a given endomorphism $f$ has only finitely many points of any given period 11 and the fact that each point in $\mathbb{P}^{N}(k)$ has at most $d$ preimages by $f$. Hence there are only finitely many possibilities for $P_{v}$ when $v \in \mathcal{V}^{\circ}$ is preperiodic.

Second, if $v \in \mathcal{V}^{\circ}$ is not preperiodic for $\Phi$, then there is some $n \geq$ 1 such that $\Phi^{n}(v) \in \mathcal{Z}$, where we note that $n$ is bounded, e.g., we certainly have $n \leq \# \mathcal{V}$. Then (45) and (46) imply that

$$
f^{n}\left(P_{v}\right)=P_{\Phi^{n}(v)}=Q_{\Phi^{n}(v)}
$$

and since we have assumed that $Q: \mathcal{Z} \rightarrow \mathbb{P}^{n}(k)$ is fixed, this determines the value of $f^{n}\left(P_{v}\right)$. Hence there are only finitely many possible values for $P_{v}$, since $f$ is at most $d$-to- 1 .

This completes the verification that the map $\Pi_{W}$ is quasi-finite, and the same is true of $\Pi_{\mathcal{P}}$, since $\Pi_{\mathcal{P}}$ is the restriction of $\Pi_{W}$ to the subscheme $\overline{\operatorname{End}}_{d}^{N}[\mathcal{P}]$ of $W$.

We next observe that the projection map

$$
\operatorname{End}_{d}^{N} \times\left(\mathbb{P}^{N}\right)^{\mathcal{V}} \longrightarrow \operatorname{End}_{d}^{N} \times\left(\mathbb{P}^{N}\right)^{\mathcal{Z}}
$$

is proper, since its fibers are products of projective spaces. Thus $\Pi_{\mathcal{P}}$, which is the restriction of this map to the closed subscheme $\overline{\operatorname{End}}_{d}^{N}[\mathcal{P}]$ of

\footnotetext{
${ }^{11}$ The points of period dividing $n$ are the points in the intersection $\Gamma_{f^{n}} \cap \Delta$, where $\Gamma_{f^{n}} \subset\left(\mathbb{P}^{N}\right)^{2}$ is the graph of $f^{n}$ and $\Delta \subset\left(\mathbb{P}^{N}\right)^{2}$ is the diagonal. Both $\Gamma_{f^{n}}$ and $\Delta$ are irreducible, so they intersect properly unless they are equal. But $\Gamma_{f^{n}}=\Delta$ implies that $f^{n}$ is the identity map, contradicting $\operatorname{deg}\left(f^{n}\right)=d^{n}>1$.
} 
$\operatorname{End}_{d}^{N} \times\left(\mathbb{P}^{N}\right)^{\mathcal{V}}$ is also proper 12 We have now shown that $\Pi_{\mathcal{P}}$ is quasifinite and proper, so [45, Chapter I, Proposition 1.10] implies that $\Pi_{\mathcal{P}}$ is finite. This completes the proof of (a) for unweighted portraits.

For a general weighted portrait $\mathcal{P}$, let $\mathcal{P}^{\prime}$ be the associated unweighted portrait. We know from Theorem 7.7 that $\overline{\operatorname{End}}_{d}^{N}[\mathcal{P}]$ is a closed subscheme of $\operatorname{End}_{d}^{N}\left[\mathcal{P}^{\prime}\right]$, and the restriction of a finite map to a closed subscheme is finite, which gives the desired result for $\operatorname{End}_{d}^{N}[\mathcal{P}]$. (b) We proved earlier in Theorem $7.7(\mathrm{~b})$ that $\operatorname{End}_{d}^{N}[\mathcal{P}]$ is $\mathrm{SL}_{N+1}$-invariant, from which it follows that its Zariski closure $\overline{\operatorname{End}}_{d}^{N}[\mathcal{P}]$ is likewise $\mathrm{SL}_{N+1}$-invariant.

(c) Every finite morphism is affine, so it follows from (a) that $\Pi_{\mathcal{P}}$ is an affine morphism. We also remark that since every geometric point of $\operatorname{End}_{d}^{N}$ has 0-dimensional $\mathrm{SL}_{N+1}$-stabilizer, the same is true for every point in $\operatorname{End}_{d}^{N} \times\left(\mathbb{P}^{N}\right)^{\mathcal{V}}$, and hence also true for every point in the subscheme $\overline{\operatorname{End}}_{d}^{N}[\mathcal{P}$ ]. We are going to use [50, Proposition 1.18], which says that if $F: X \rightarrow Y$ is a quasi-affine morphism commuting with the action of a reductive group $G$, and if $\mathcal{L}$ is a $G$-linearized invertible sheaf on $Y$, then

$$
F^{-1}\left(\left\{\begin{array}{c}
G \text {-stable points of } Y \\
\text { relative to } \mathcal{L}
\end{array}\right\}\right) \subseteq\left\{\begin{array}{c}
G \text {-stable points of } X \\
\text { relative to } F^{*} \mathcal{L}
\end{array}\right\}
$$

Using notation from Corollary 8.3, we have

$$
n=m_{\Sigma}=\# \mathcal{Z}=\#\left(\mathcal{V} \backslash \mathcal{V}^{\circ}\right) \text { and } m_{0}=m,
$$

so our assumption (42) says that $m_{0}>m_{\Sigma} /(d-1)$. This is exactly what is required to apply Corollary 8.3, so we conclude that

$$
\operatorname{End}_{d}^{N} \times\left(\mathbb{P}^{N}\right)^{\mathcal{Z}} \text { is } \mathrm{SL}_{N+1} \text {-stable for } \mathcal{O}(m, 1, \ldots, 1) .
$$

Applying this, we find that

$$
\begin{aligned}
& \overline{\operatorname{End}}_{d}^{N}[\mathcal{P}]=\Pi_{\mathcal{P}}^{-1}\left(\operatorname{End}_{d}^{N} \times\left(\mathbb{P}^{N}\right)^{\mathcal{Z}}\right) \\
&\left.=\Pi_{\mathcal{P}}^{-1}\left(\left\{\mathrm{SL}_{N+1} \text {-stable points of } \operatorname{End}_{d}^{N} \times\left(\mathbb{P}^{N}\right)^{\mathcal{Z}}\right\}\right) \quad \text { from (48) }\right) \\
& \subseteq\left\{\mathrm{SL}_{N+1} \text {-stable points of } \overline{\operatorname{End}}_{d}^{N}[\mathcal{P}]\right\} \quad \text { from (47]) } \\
& \text { with } G=\mathrm{SL}_{N+1}, \mathcal{L}=\mathcal{O}_{\operatorname{End}_{d}^{N} \times\left(\mathbb{P}^{N}\right)^{\mathcal{Z}}(m, 1, \ldots, 1),} \\
& \quad F=\Pi_{\mathcal{P}}, X=\overline{\operatorname{End}}_{d}^{N}[\mathcal{P}], Y=\operatorname{End}_{d}^{N} \times\left(\mathbb{P}^{N}\right)^{\mathcal{Z}} .
\end{aligned}
$$

Hence $\overline{\operatorname{End}}_{d}^{N}[\mathcal{P}]$ is contained in the $\mathrm{SL}_{N+1}$-stable locus of $\mathbb{P}^{M} \times\left(\mathbb{P}^{N}\right)^{\mathcal{V}}$, which completes the proof of Theorem 10.1 .

\footnotetext{
${ }^{12}$ This follows from: (1) If $Z$ is a closed subscheme of $X$, then the inclusion $Z \hookrightarrow X$ is proper. (2) A composition of proper morphisms is proper. Both (1) and (2) are exercises using the valuative criterion, or see [28, II.4.8].
} 
Combining Theorem 9.1 with Theorem 14.2, a result that we will prove later, leads to a useful fact about formal models for unweighted portraits.

Corollary 9.3. Let $\mathcal{P}=\left(\mathcal{V}^{\circ}, \mathcal{V}, \Phi\right)$ be an unweighted portrait such that $\operatorname{End}_{d}^{N}[\mathcal{P}]$ is non-empty, and let $k$ be an algebraically closed field of characteristic 0 . Then every $f \in \operatorname{End}_{d}^{N}(k)$ admits a formal model for the portrait $\mathcal{P}$ over the field $k$. This is true even if one first arbitrarily assigns values in $\mathbb{P}^{N}(k)$ to the vertices in $\mathcal{V} \backslash \mathcal{V}^{\circ}$.

Proof. As before, we let $\mathcal{Z}:=\mathcal{V} \backslash \mathcal{V}^{\circ}$. We first note that

$$
\begin{aligned}
\operatorname{dim} \operatorname{End}_{d}^{N} \times\left(\mathbb{P}^{N}\right)^{\mathcal{Z}} & =\operatorname{dim} \operatorname{End}_{d}^{N}+N \cdot \# \mathcal{Z} \\
& =\operatorname{dim} \operatorname{End}_{d}^{N}[\mathcal{P}] \text { from Theorem } 14.2(\mathrm{~d}), \\
& =\operatorname{dim} \overline{\operatorname{End}}_{d}^{N}[\mathcal{P}] \text { since } \overline{\operatorname{End}}_{d}^{N}[\mathcal{P}] \text { is the Zariski } \\
& \text { closure of } \operatorname{End}_{d}^{N}[\mathcal{P}] .
\end{aligned}
$$

Next we use the fact from Theorem 9.1(a) that

$$
\Pi_{\mathcal{P}}: \overline{\operatorname{End}}_{d}^{N}[\mathcal{P}] \rightarrow \operatorname{End}_{d}^{N} \times\left(\mathbb{P}^{N}\right)^{\mathcal{Z}}
$$

is finite, so in particular it is proper, so the image $\Pi_{\mathcal{P}}\left(\overline{\operatorname{End}}_{d}^{N}[\mathcal{P}]\right)$ is a closed subscheme of $\operatorname{End}_{d}^{N} \times\left(\mathbb{P}^{N}\right)^{\mathcal{Z}}$. Thus the image is both closed and has dimension equal to the dimension of the irreducible variety $\operatorname{End}_{d}^{N} \times\left(\mathbb{P}^{N}\right)^{\mathcal{Z}}$, hence the image is all of $\operatorname{End}_{d}^{N} \times\left(\mathbb{P}^{N}\right)^{\mathcal{Z}}$.

Example 9.4. Theorem 9.1 says in particular that if $\mathcal{P}$ is a preperiodic portrait, then the map $\overline{\operatorname{End}}_{d}^{N}[\mathcal{P}] \rightarrow \operatorname{End}_{d}^{N}$ is finite and the map $\operatorname{End}_{d}^{N}[\mathcal{P}] \rightarrow \operatorname{End}_{d}^{N}$ is quasi-finite. We note that the latter map need not be finite, since it need not be proper. To illustrate, let $\mathcal{P}$ be the portrait consisting of two fixed points, and consider the family of maps

$$
t \longmapsto f_{t}:=z(z-t+1) \in \operatorname{End}_{2}^{1},
$$

valid for all $t$. On the set $t \neq 0$, we can extend this to a family

$$
t \longmapsto\left(f_{t}, 0, t\right) \in \operatorname{End}_{2}^{1}[\mathcal{P}]
$$

but we clearly cannot extend to $t=0$, since $\operatorname{End}_{2}^{1}[\mathcal{P}]$ classifies triples $(f, P, Q)$ with $f(P)=P, f(Q)=Q$, and $P \neq Q$, so $\left(f_{0}, 0,0\right)$ is not a point of $\operatorname{End}_{2}^{1}[\mathcal{P}]$.

Example 9.5. Let $W$ be the scheme defined by (43). We proved that $\overline{\operatorname{End}}_{d}^{N}[\mathcal{P}]$ is contained in $W$, but we want to give an example showing that they need not be equal. Let $\mathcal{P}$ be the portrait consisting of two fixed points. Then $W$ contains the subscheme

$$
\{(f, P, Q): P=Q \text { and } f(P)=P\}
$$


which is isomorphic to $\operatorname{End}_{d}^{N}\left[1\right.$ fixed point]. But $(f, P, P) \in \overline{\operatorname{End}}_{d}^{N}[\mathcal{P}]$ if and only if $P$ is a fixed point of multiplicity strictly larger than 1 . For example, on $\mathbb{P}^{1}$ we have

$$
(f, P, P) \in \overline{\operatorname{End}}_{d}^{1}[\mathcal{P}] \quad \Longleftrightarrow \quad f^{\prime}(P)=1,
$$

where we write $f^{\prime}(P)$ for the multiplier of $f$ at $P$. Thus for $N=1$, the scheme $W$ has two irreducible components, and for higher values of $N$ and/or more complicated portraits $\mathcal{P}$, it may have many more components.

\section{The Moduli Space $\mathcal{M}_{d}^{N}[\mathcal{P}]$ for Portrait Models}

In this section we construct moduli spaces for dynamical systems on $\mathbb{P}^{N}$ with a specified portrait.

Theorem 10.1. Continuing with the notation from Theorem 9.1, we assume as in that theorem that $m$ is an integer satisfying

$$
m>\frac{1}{d-1} \#\left(\mathcal{V} \backslash \mathcal{V}^{\circ}\right)
$$

(a) There are well-defined geometric quotient schemes 13

$$
\begin{aligned}
& \overline{\mathcal{M}}_{d}^{N}[\mathcal{P}]:=\overline{\operatorname{End}}_{d}^{N}[\mathcal{P}] / / \mathrm{SL}_{N+1}, \\
& \mathcal{M}_{d}^{N}[\mathcal{P}]:=\operatorname{End}_{d}^{N}[\mathcal{P}] / / \mathrm{SL}_{N+1},
\end{aligned}
$$

satisfying:

(i) The structure sheaf of $\overline{\mathcal{M}}_{d}^{N}[\mathcal{P}]$ is the maximal subsheaf of $\Pi_{\mathcal{P}}^{*} \mathcal{O}_{\mathbb{P}^{M} \times\left(\mathbb{P}^{N}\right) \mathcal{V} \backslash \mathcal{V}^{\circ}}(m, 1, \ldots, 1)$ that is $\mathrm{SL}_{N+1}$-invariant.

(ii) $\overline{\mathcal{M}}_{d}^{N}[\mathcal{P}]$ is proper over $\mathcal{M}_{d}^{N}$.

(iii) $\mathcal{M}_{d}^{N}[\mathcal{P}]$ is an open subscheme of $\overline{\mathcal{M}}_{d}^{N}[\mathcal{P}]$.

(b) The scheme $\mathcal{M}_{d}^{N}[\mathcal{P}]$ is a coarse moduli space for the $(N, d, \mathcal{P})$ moduli problem.

(c) Let $\mathcal{P}_{1}$ and $\mathcal{P}_{2}$ be portraits, and assume that $m$ satisfies (49) for both $\mathcal{P}_{1}$ and $\mathcal{P}_{2}$. Then the map described in Proposition 7.8 descends to give a natural map

$$
\operatorname{Hom}\left(\mathcal{P}_{1}, \mathcal{P}_{2}\right) \longrightarrow \operatorname{Hom}\left(\mathcal{M}_{d}^{N}\left[m, \mathcal{P}_{2}\right], \mathcal{M}_{d}^{N}\left[m, \mathcal{P}_{1}\right]\right) \text {. }
$$

N.B. In order to obtain a map between the two moduli spaces, it is necessary to use the same $m$ for both.

\footnotetext{
${ }^{13}$ If we need to indicate the dependence of the structure sheaf on $m$, for example as in Theorem 10.1 (c), then we write $\overline{\mathcal{M}}_{d}^{N}[m, \mathcal{P}]$ and $\mathcal{M}_{d}^{N}[m, \mathcal{P}]$.
} 
Definition 10.2. We set the convenient notation

$$
\langle\cdot\rangle: \operatorname{End}_{d}^{N}[\mathcal{P}] \longrightarrow \mathcal{M}_{d}^{N}[\mathcal{P}]
$$

for the quotient map, i.e., if $(f, P) \in \operatorname{End}_{d}^{N}[\mathcal{P}]$ is a model for $\mathcal{P}$, then $\langle f, P\rangle \in \mathcal{M}_{d}^{N}[\mathcal{P}]$ denotes the $\mathrm{SL}_{N+1}$-equivalence class of $(f, P)$.

Remark 10.3. One can define interesting subschemes of $\mathcal{M}_{d}^{N}[\mathcal{P}]$ by specifying that the automorphism group of the map $f$ in the pair $(f, \boldsymbol{P})$ has a specified subgroup. Thus for each finite group $\mathcal{G}$, we define14

$$
{ }_{\mathcal{G}} \mathcal{M}_{d}^{N}[\mathcal{P}]:=\left\{(f, \boldsymbol{P}) \in \mathcal{M}_{d}^{N}[\mathcal{P}]: \begin{array}{l}
\text { Aut }(f) \text { contains a sub- } \\
\text { group isomorphic to } \mathcal{G}
\end{array}\right\} .
$$

Levy [38] proves that ${ }_{\mathcal{G}} \mathcal{M}_{d}^{N}={ }_{\mathcal{G}} \mathcal{M}_{d}^{N}[\emptyset]$ is a Zariski closed subvariety of $\mathcal{M}_{d}^{N}$ for all $\mathcal{G}$, and that it is non-empty for at most finitely many $\mathcal{G}$, hence the same is true for ${ }_{\mathcal{G}} \mathcal{M}_{d}^{N}[\mathcal{P}] \subseteq \mathcal{M}_{d}^{N}[\mathcal{P}]$.

A nice example is $\mathbb{Z} / 2 \mathbb{Z} \mathcal{M}_{2}^{1}[\emptyset]$, which is a cuspidal cubic curve in $\mathcal{M}_{2}^{1}[\emptyset]=\mathcal{M}_{2}^{1} \cong \mathbb{A}^{2}$. The cusp is the unique point in $\mathcal{S}_{3} \mathcal{M}_{2}^{1}[\emptyset]$. Let $p$ be prime, and let $\mathcal{C}_{n}$ denote the unweighted portrait consisting of a single $n$-cycle. Then Manes [42] shows that the moduli space $\mathbb{Z} / p \mathbb{Z} \mathcal{M}_{d}^{1}\left[\mathcal{C}_{p \ell}\right]$ is reducible for infinitely many primes $\ell$. See also [43] for a catalog of the schemes ${ }_{\mathcal{G}} \mathcal{M}_{2}^{2}[\emptyset]$ for groups satisfying $\# \mathcal{G} \geq 3$. For example, the largest group for which $\mathcal{G}_{\mathcal{2}}^{2}[\emptyset]$ is non-empty is the group $\mathcal{G}=(\mathbb{Z} / 7 \mathbb{Z}) \rtimes(\mathbb{Z} / 3 \mathbb{Z})$ of order 21.

Proof of Theorem 10.1. (a) This follows directly from Theorem 9.1, combined with standard facts from geometric invariant theory regarding geometric quotients of schemes of stable points.

(b) This is immediate from the fact that $\operatorname{End}_{d}^{N}[\mathcal{P}]$ is a universal parameter space for models of $\mathcal{P}$, combined with the fact that $\mathcal{M}_{d}^{N}[\mathcal{P}]$ is a geometric quotient of $\operatorname{End}_{d}^{N}[\mathcal{P}]$ for the action of $\mathrm{SL}_{N+1}$ that matches the equivalence relation on models of $\mathcal{P}$ used to define the $(N, d, \mathcal{P})$ moduli problem.

(c) Proposition 7.8 says that every portrait morphism $\alpha: \mathcal{P}_{1} \rightarrow \mathcal{P}_{2}$ induces a scheme-theoretic morphism of the associated parameter spaces

$$
\alpha^{*}: \operatorname{End}_{d}^{N}\left[\mathcal{P}_{2}\right] \stackrel{\sim}{\longrightarrow} \operatorname{End}_{d}^{N}\left[\mathcal{P}_{1}\right]
$$

via $\alpha^{*}(f, \boldsymbol{P})=(f, \boldsymbol{P} \circ \alpha)$. Further, for $\varphi \in \mathrm{SL}_{N+1}$ and $(f, \boldsymbol{P}) \in$ $\operatorname{End}_{d}^{N}\left[\mathcal{P}_{2}\right]$ we have

$$
\alpha^{*} \circ \varphi\left(f,\left(P_{v}\right)_{v \in \mathcal{V}}\right)=\alpha^{*}\left(f^{\varphi},\left(\varphi^{-1} P_{v}\right)_{v \in \mathcal{V}}\right)=\left(f^{\varphi},\left(\varphi^{-1} P_{\alpha(v)}\right)_{v \in \mathcal{V}}\right),
$$

\footnotetext{
${ }^{14}$ We apologize for this clumsy notation, which will not be used further in this paper, but we want to keep $\mathcal{G}$ separate from the $\operatorname{subgroup} \mathcal{A}$ of $\operatorname{Aut}(\mathcal{P})$ that appears in the notation defined in Section 10.1
} 


$$
\varphi \circ \alpha^{*}\left(f,\left(P_{v}\right)_{v \in \mathcal{V}}\right)=\varphi\left(f,\left(P_{\alpha(v)}\right)_{v \in \mathcal{V}}\right)=\left(f^{\varphi},\left(\varphi^{-1} P_{\alpha(v)}\right)_{v \in \mathcal{V}}\right) .
$$

Thus the action of $\alpha^{*}$ commutes with the action of $\mathrm{SL}_{N+1}$, so $\alpha^{*}$ descends to a morphism of the quotient schemes. (We should also point out that $\alpha^{*}$ preserves $\mathcal{O}(m, 1, \ldots, 1)$, since it simply permutes the factors of $\mathbb{P}^{N}$.)

10.1. The Moduli Space $\mathcal{M}_{d}^{N}[\mathcal{P} \mid \mathcal{A}]$ for Portraits Modulo Automorphisms. Applying Theorem [10.1](c) with $\mathcal{P}_{1}=\mathcal{P}_{2}=\mathcal{P}$, we see that every automorphism of an unweighted portrait induces a schemetheoretic automorphism of the associated moduli space $\mathcal{M}_{d}^{N}[\mathcal{P}]$. The group $\operatorname{Aut}(\mathcal{P})$ is finite, since it is a subgroup of the symmetric group on the set $\mathcal{V} 15$ Quotients of schemes by finite groups of automorphisms always exist as GIT geometric quotients. Hence for any subgroup $\mathcal{A} \subset \operatorname{Aut}(\mathcal{P})$, we obtain a GIT quotient space. We formally state this result as a corollary.

Corollary 10.4. Let $\mathcal{P}$ be a portrait, let $\mathcal{A} \subseteq \operatorname{Aut}(\mathcal{P})$ be a subgroup of the automorphism group of $\mathcal{P}$. Then the natural action of $\mathcal{A}$ on $\operatorname{End}_{d}^{N}[\mathcal{P}]$ commutes with the action of $\mathrm{SL}_{N+1}$, and hence there exists a GIT geometric quotient space

$$
\mathcal{M}_{d}^{N}[\mathcal{P} \mid \mathcal{A}]:=\operatorname{End}_{d}^{N}[\mathcal{P}] / /\left(\mathcal{A} \cdot \mathrm{SL}_{N+1}\right) \cong \mathcal{M}_{d}^{N}[\mathcal{P}] / / \mathcal{A}
$$

Definition 10.5. With $\mathcal{M}_{d}^{N}[\mathcal{P} \mid \mathcal{A}]$ as defined in Corollary 10.4, we set the following notation 16

$$
\mathcal{M}_{d}^{N}[\mathcal{P} \mid 1]:=\mathcal{M}_{d}^{N}[\mathcal{P}] \text { and } \mathcal{M}_{d}^{N}[\mathcal{P} \mid 0]:=\mathcal{M}_{d}^{N}[\mathcal{P} \mid \operatorname{Aut}(\mathcal{P})] .
$$

By analogy with theorems and conjectures on moduli spaces of curves and abelian varieties, we make the following conjecture. See Theorem $\left[13.3\right.$ (c) for an example of a $\mathcal{M}_{d}^{1}[\mathcal{P}]$ that is a surface of general type.

Conjecture 10.6. Let $N \geq 1$ and $d \geq 2$.

(a) There is a constant $C_{1}(N, d)$ so that for all unweighted preperiodic portraits $\mathcal{P}=(\mathcal{V}, \Phi)$ satisfying $\# \mathcal{V} \geq C_{1}(N, d)$ and $\mathcal{M}_{d}^{N}[\mathcal{P}] \neq \emptyset$, the variety $\mathcal{M}_{d}^{N}[\mathcal{P}] \otimes_{\mathbb{Z}} \mathbb{C}$ is an irreducible variety of general type.

(b) There is a constant $C_{0}(N, d)$ so that for all unweighted preperiodic portraits $\mathcal{P}=(\mathcal{V}, \Phi)$ satisfying $\# \mathcal{V} \geq C_{0}(N, d)$ and $\mathcal{M}_{d}^{N}[\mathcal{P} \mid 0] \neq \emptyset$, the variety $\mathcal{M}_{d}^{N}[\mathcal{P} \mid 0] \otimes_{\mathbb{Z}} \mathbb{C}$ is an irreducible variety of general type.

\footnotetext{
${ }^{15}$ We generally assume that $\mathcal{V} \neq \emptyset$, and in any case we define the symmetry group of the empty set to be the trivial group.

${ }^{16}$ This notation is motivated by the notation for the elliptic modular curves $X_{1}(n)$ and $X_{0}(n)$, where $X_{1}(n)$ classifies pairs $(E, P)$ consisting of an elliptic curve and a point of exact order $n$, and $X_{0}(n)=X_{1}(n) / /(\mathbb{Z} / n \mathbb{Z})^{*}$, the action of $k \in(\mathbb{Z} / n \mathbb{Z})^{*}$ being to send $(E, P)$ to $(E, k P)$.
} 
10.2. The Moduli Space for Maps with Points of Exactly Specified Multiplicities. In certain situations one may wish to classify maps $f$ and points $P_{i}$ whose multiplicities (ramification indices) are specified exactly, rather than by inequalities. See for example Section 18 and [56] for applications with $N=1$. We start with a definition.

Definition 10.7. We put a partial order on the set of weighted portraits by saying that $\mathcal{P}^{\prime} \geq \mathcal{P}$ if there is a portrait morphism $\alpha: \mathcal{P} \rightarrow \mathcal{P}^{\prime}$ that is a bijection on vertices. Referring to Definition 3.4 and writing $\mathcal{P}=\left(\mathcal{V}^{\circ}, \mathcal{V}, \Phi, \epsilon\right)$ and $\mathcal{P}^{\prime}=\left(\mathcal{V}^{\prime \circ}, \mathcal{V}^{\prime}, \Phi^{\prime}, \epsilon^{\prime}\right)$, this means that $\alpha$ is a bijection $\alpha: \mathcal{V} \stackrel{\sim}{\longrightarrow} \mathcal{V}^{\prime}$ satisfying

$$
\alpha\left(\mathcal{V}^{\circ}\right)=\mathcal{V}^{\prime \circ}, \quad \alpha \circ \Phi=\Phi^{\prime} \circ \alpha, \quad \text { and } \quad \epsilon^{\prime} \circ \alpha \geq \epsilon .
$$

The intuition is that $\mathcal{P}^{\prime} \geq \mathcal{P}$ if $\mathcal{P}$ and $\mathcal{P}^{\prime}$ have the same vertices and the same maps, but the weights assigned to the vertices in $\mathcal{P}^{\prime}$ are allowed to be greater than the weights assigned in $\mathcal{P}$.

Proposition 10.8. Let $N \geq 1$ and $d \geq 2$, and let $\mathcal{P}$ be a portrait.

(a) Let $\mathcal{P}^{\prime} \geq \mathcal{P}$. Then the map $\alpha: \mathcal{P} \rightarrow \mathcal{P}^{\prime}$ induces an isomorphism of $\operatorname{End}_{d}^{\bar{N}}\left[\mathcal{P}^{\prime}\right]$ with a closed subscheme of $\operatorname{End}_{d}^{N}[\mathcal{P}]$, and similarly allows us to view $\mathcal{M}_{d}^{N}\left[\mathcal{P}^{\prime}\right]$ as a closed subscheme of $\mathcal{M}_{d}^{N}[\mathcal{P}]$.

(b) For a given $(N, d)$, there are only finitely many portraits $\mathcal{P}^{\prime}$ satisfying $\mathcal{P}^{\prime} \geq \mathcal{P}$ and $\operatorname{End}_{d}^{N}\left[\mathcal{P}^{\prime}\right] \neq \emptyset$.

Proof. (a) We identify the vertices and maps of $\mathcal{P}$ and $\mathcal{P}^{\prime}$, say $\mathcal{P}=$ $\left(\mathcal{V}^{\circ}, \mathcal{V}, \Phi, \epsilon\right)$ and $\mathcal{P}^{\prime}=\left(\mathcal{V}^{\circ}, \mathcal{V}, \Phi, \epsilon^{\prime}\right)$. We also let $\mathcal{P}^{\prime \prime}=\left(\mathcal{V}^{\circ}, \mathcal{V}, \Phi, 1\right)$ be the associated unweighted portrait, i.e., it has the same vertices and map as $\mathcal{P}$ and $\mathcal{P}^{\prime}$, but every vertex in $\mathcal{V}^{\circ}$ has weight 1 . Then Theorem 7.7 tells us that $\operatorname{End}_{d}^{N}[\mathcal{P}]$ and $\operatorname{End}_{d}^{N}\left[\mathcal{P}^{\prime}\right]$ are closed subschemes of $\operatorname{End}_{d}^{N}\left[\mathcal{P}^{\prime \prime}\right]$ whose geometric points are specified by the multiplicity condition (10) appearing in Theorem 7.7. Since $\epsilon^{\prime} \geq \epsilon$ by assumption, we see from (10) that $\operatorname{End}_{d}^{N}\left[\mathcal{P}^{\prime}\right] \subseteq \operatorname{End}_{d}^{N}[\mathcal{P}]$, and then taking $\mathrm{SL}_{N+1^{-}}$ quotients as in Theorem 10.1 gives the analogous inclusion $\mathcal{M}_{d}^{N}\left[\mathcal{P}^{\prime}\right] \subseteq$ $\mathcal{M}_{d}^{N}[\mathcal{P}]$.

(b) For any $Q \in \mathbb{P}^{N}$, the multiplicities of the points in the inverse image of $Q$ satisfy

$$
\sum_{P \in f^{-1}(Q)} e_{f}(P)=(\text { topological degree of } f)=\operatorname{deg}(f)^{N},
$$

so taking $Q=f(P)$ yields $e_{f}(P) \leq \operatorname{deg}(f)^{N}$. Hence if $\operatorname{End}_{d}^{N}\left[\mathcal{P}^{\prime}\right] \neq$ $\emptyset$, then the weight of every vertex $v^{\prime}$ in $\mathcal{P}^{\prime}$ must satisfy $\epsilon^{\prime}\left(v^{\prime}\right) \leq d^{N}$, and since $\mathcal{V}^{\prime}=\alpha(\mathcal{V})$, there are a fixed number of vertices in $\mathcal{V}^{\prime}$ to 
which weights may be assigned. Hence there are only finitely many possibilities.

Definition 10.9. For $N \geq 1$ and $d \geq 2$, we define

$$
\begin{aligned}
\operatorname{End}_{d}^{N}[\mathcal{P}]^{\circ} & =\operatorname{End}_{d}^{N}[\mathcal{P}] \backslash \bigcup_{\mathcal{P}^{\prime} \geq \mathcal{P}, \mathcal{P}^{\prime} \neq \mathcal{P}} \operatorname{End}_{d}^{N}\left[\mathcal{P}^{\prime}\right], \\
\mathcal{M}_{d}^{N}[\mathcal{P}]^{\circ} & =\mathcal{M}_{d}^{N}[\mathcal{P}] \backslash \bigcup_{\mathcal{P}^{\prime} \geq \mathcal{P}, \mathcal{P}^{\prime} \neq \mathcal{P}} \mathcal{M}_{d}^{N}\left[\mathcal{P}^{\prime}\right] .
\end{aligned}
$$

It follows from Proposition 10.8 that $\operatorname{End}_{d}^{N}[\mathcal{P}]^{\circ}$ is an open subscheme of $\operatorname{End}_{d}^{N}[\mathcal{P}]$ and that $\mathcal{M}_{d}^{N}[\mathcal{P}]^{\circ}$ is an open subscheme of $\mathcal{M}_{d}^{N}[\mathcal{P}]$.

\section{Refined Stability Criteria for $\mathbb{P}^{1}$}

Corollary 8.3 gives a sufficient numerical criterion for $\operatorname{End}_{d}^{N} \times\left(\mathbb{P}^{N}\right)^{n}$ to be $\mathrm{SL}_{N+1}$-stable for the line bundle $\mathcal{O}(\boldsymbol{m}):=\mathcal{O}\left(m_{0}, \ldots, m_{n}\right)$. We used this in Theorem 9.1 to prove that $\overline{\operatorname{End}}_{d}^{N}[\mathcal{P}]$ is $\mathrm{SL}_{N+1}$-stable for the pull-back of $\mathcal{O}(\boldsymbol{m})$, where we recall that $\overline{\operatorname{End}}_{d}^{N}[\mathcal{P}]$ is the Zariski closure of $\operatorname{End}_{d}^{N}[\mathcal{P}]$ in $\operatorname{End}_{d}^{N} \times\left(\mathbb{P}^{N}\right)^{n}$. In particular, the scheme $\overline{\operatorname{End}}_{d}^{n}[\mathcal{P}]$ may include geometric points $(f, \boldsymbol{P})$ in which $\boldsymbol{P}$ lies on the big diagonal, i.e., the $n$ points in $\boldsymbol{P}=\left(P_{1}, \ldots, P_{n}\right)$ need not be distinct.

In this section we consider maps on $\mathbb{P}^{1}$, i.e., we take $N=1$, and we restrict to points $\boldsymbol{P}$ lying on the complement of the big diagonal. In this case, we give a refined description of the $\mathrm{SL}_{2}$-stable locus of $\operatorname{End}_{d}^{1}[\mathcal{P}]$ relative to $\mathcal{O}(m, 1, \ldots, 1)$ with $m \in\{1,2\}$, and we construct the associated moduli spaces.

Proposition 11.1. Let $d \geq 2$, let $n \geq 0$, and let $\left(\mathbb{P}^{1}\right)_{\Delta}^{n}$ be the complement of the big diagonal as in Section 7.1 .

(a) $\operatorname{End}_{d}^{1} \times\left(\mathbb{P}^{1}\right)_{\Delta}^{n}$ is $\mathrm{SL}_{2}$-semistable relative to $\mathcal{O}(1,1, \ldots, 1)$.

(b) $\operatorname{End}_{d}^{1} \times\left(\mathbb{P}^{1}\right)_{\Delta}^{n}$ is $\mathrm{SL}_{2}$-stable relative to $\mathcal{O}(1,1, \ldots, 1)$ except for the case $(d, n)=(2,1)$.

(c) $\operatorname{End}_{2}^{1} \times \mathbb{P}^{1}$ is $\mathrm{SL}_{2}$-stable relative to $\mathcal{O}(2,1)$. For the sheaf $\mathcal{O}(1,1)$, we have

$$
\begin{aligned}
\left\{(f, P) \in \operatorname{End}_{2}^{1} \times \mathbb{P}^{1}:(f, P)\right. \text { is } & \left.\mathrm{SL}_{2} \text {-stable relative to } \mathcal{O}(1,1)\right\} \\
& =\left\{(f, P) \in \operatorname{End}_{2}^{1} \times \mathbb{P}^{1}: f(P) \neq P\right\} .
\end{aligned}
$$

Proof. For $n=0$, we already know that $\operatorname{End}_{d}^{1}$ is stable, so we assume henceforth that $n \geq 1$. We are going to apply Theorem 8.1 with $N=1$ and $\boldsymbol{m}=(1,1, \ldots, 1)$. In particular, $m_{0}=1$ and $m_{\Sigma}=n$. The only proper linear subspaces $L \subsetneq \mathbb{P}^{1}$ consist of single points, say $L_{Q}=\{Q\}$. We are only considering pairs $(f, \boldsymbol{P})$ where $\boldsymbol{P}=\left(P_{1}, \ldots, P_{n}\right)$ consists 
of $n$ distinct points, so for a given $L_{Q}$, there is at most one $\nu$ with $P_{\nu} \in$ $L_{Q}$. Thus

$$
C_{\boldsymbol{P}}\left(L_{Q}\right)= \begin{cases}1 & \text { if } Q=P_{\nu} \text { for some } \nu, \\ 0 & \text { otherwise. }\end{cases}
$$

(We are using the fact that every $m_{\nu}=1$.) We also note that the definition (19) of $D_{\epsilon}(L)$ for these parameters gives

$$
D_{0}\left(L_{Q}\right)=\frac{n+d-1}{2} \geq 1
$$

where the lower bound comes from our assumption that $d \geq 2$ and $n \geq 1$. Hence the estimate

$$
C_{\boldsymbol{P}}\left(L_{Q}\right) \leq D_{0}\left(L_{Q}\right)
$$

is always true, so Theorem 8.1 (a) tells us that $(f, \boldsymbol{P})$ is semistable relative to $\mathcal{O}(1,1, \ldots, 1)$, which proves (a). Further, if $(d, n) \neq(2,1)$, then $D_{0}\left(L_{Q}\right) \geq 3 / 2$, so (50) is a strict inequality, and Theorem 8.1 (b) tells us that $(f, \boldsymbol{P})$ is stable relative to $\mathcal{O}(1,1, \ldots, 1)$. This proves (b).

It remains to consider stability in the case that $(d, n)=(2,1)$ for the invertible sheaf $\mathcal{O}(1,1)$. Thus $(f, P)$ consists of a map and a single point, and we need to compute $\mu^{\mathcal{O}(1,1)}((f, P), \ell)$ for non-trivial 1parameter subgroups $\ell$. After a change of coordinates, we may assume that

$$
\ell(\alpha):=\left(\begin{array}{cc}
\alpha^{-1} & 0 \\
0 & \alpha
\end{array}\right)
$$

We write $P=[U(0), U(1)]$ and

$$
f=\left[a_{0}(2) X^{2}+a_{0}(1) X Y+a_{0}(0) Y^{2}, a_{1}(2) X^{2}+a_{1}(1) X Y+a_{1}(0) Y^{2}\right],
$$

which is an abbreviated version of the notation used in the proof of Theorem 8.1 .

A basis of $\ell$-diagonalized global sections of $\mathcal{O}(1,1)$ on $\operatorname{End}_{2}^{1} \times \mathbb{P}^{1}$ is given by

$$
\left\{a_{\rho}(e) U(i): 0 \leq \rho \leq 1,0 \leq e \leq 2,0 \leq i \leq 1\right\}
$$

and the action of $\ell$ on these sections is via

$$
\ell \star a_{\rho}(e) U(i)=\alpha^{2(2-e-\rho-i)} a_{\rho}(e) U(i) .
$$

Hence

$$
\mu^{\mathcal{O}(1,1)}((f, P), \ell)=\max _{a_{\rho}(e) U(i) \neq 0} 2(e+\rho+i-2) .
$$

Suppose first that $a_{1}(2) \neq 0$. Then using the sections $a_{1}(2) U(i)$ gives

$$
\mu^{\mathcal{O}(1,1)}((f, P), \ell) \geq \begin{cases}2(2+1+0-2)=2 & \text { if } U(0) \neq 0 \\ 2(2+1+1-2)=4 & \text { if } U(1) \neq 0\end{cases}
$$


But $U(0)$ and $U(1)$ cannot both be 0 , so $\mu^{\mathcal{O}(1,1)}((f, P), \ell)>0$, and the numerical criterion (Theorem 4.3) tells us that $(f, P)$ is stable.

We are reduced to the case that $a_{1}(2)=0$. This implies that $a_{0}(2) \neq$ 0 , since otherwise $f$ would not be a morphism at $[0,1]$. Looking at the sections $a_{0}(2) U(i)$ yields

$$
\mu^{\mathcal{O}(1,1)}((f, P), \ell) \geq \begin{cases}2(2+0+0-2)=0 & \text { if } U(0) \neq 0 \\ 2(2+0+1-2)=2 & \text { if } U(1) \neq 0\end{cases}
$$

Hence if $U(1) \neq 0$, we again conclude that $(f, P)$ is stable. And we note that since $a_{1}(2)=0$, the condition $U(1)=0$ implies that $f(P)=P$, so this shows that if $f(P) \neq P$, then $(f, P)$ is stable.

Finally, suppose that $f(P)=P$. After a change of variables we may assume $P=[1,0]$, hence we also have $a_{1}(2)=0$. We now take the 1 -parameter subgroup $\ell$ given in (51) and compute $\mu^{\mathcal{O}(1,1)}((f, P), \ell)$. There are five $\ell$-diagonalized sections that are potentially non-zero, as listed in the following table with their corresponding values of $e+\rho+$ $i-2$ :

\begin{tabular}{|c||c|c|c|c|c|}
\hline Section & $U(0) a_{0}(2)$ & $U(0) a_{0}(1)$ & $U(0) a_{0}(0)$ & $U(0) a_{1}(1)$ & $U(0) a_{1}(0)$ \\
\hline$e+\rho+i-2$ & 0 & -1 & -2 & 0 & -1 \\
\hline
\end{tabular}

Hence

$$
\mu^{\mathcal{O}(1,1)}((f, P), \ell) \leq \max \{0,-1,-2,0,-1\}=0,
$$

so $(f, P)$ is not stable. This completes the proof that if the pair $(f, P) \in \operatorname{End}_{2}^{1} \times \mathbb{P}^{1}$ satisfies $f(P)=P$, then $(f, P)$ is not stable relative to $\mathcal{O}(1,1)$.

Corollary 11.2. Let $\mathcal{P}=\left(\mathcal{V}^{\circ}, \mathcal{V}, \Phi, \epsilon\right)$ be a portrait, let $d \geq 2$, and let $\mathcal{L}=\mathcal{O}(1,1, \ldots, 1)$ unless $d=2$ and $\#\left(\mathcal{V} \backslash \mathcal{V}^{\circ}\right)=1$, in which case let $\mathcal{L}=\mathcal{O}(2,1)$. Then $\operatorname{End}_{d}^{1}[\mathcal{P}]$ is $\mathrm{SL}_{2}$-stable relative to $\Pi_{\mathcal{P}}^{*} \mathcal{L}$, and hence there is a GIT moduli quotient scheme

$$
\mathcal{M}_{d}^{1}[\mathcal{P}]:=\operatorname{End}_{d}^{1}[\mathcal{P}] / / \mathrm{SL}_{2} \quad \text { with structure sheaf }\left(\Pi_{\mathcal{P}}^{*} \mathcal{L}\right)^{\mathrm{SL}_{2}} .
$$

Proof. We follow the proof of Theorem 9.1(c), mutatis mutandis, but using $\operatorname{End}_{d}^{1}$ instead of $\overline{\mathrm{End}}_{d}^{1}$, since Proposition 11.1 only gives stability of $\operatorname{End}_{d}^{1} \times\left(\mathbb{P}^{1}\right)_{\Delta}^{n}$, i.e., on the complement of the big diagonal. Thus

$$
\begin{aligned}
& \operatorname{End}_{d}^{1}[\mathcal{P}]=\Pi_{\mathcal{P}}^{-1}\left(\operatorname{End}_{d}^{1} \times\left(\mathbb{P}^{1}\right)_{\Delta}^{\mathcal{Z}}\right) \\
& \subseteq \Pi_{\mathcal{P}}^{-1}\left(\mathrm{SL}_{N+1} \text {-stable points of } \operatorname{End}_{d}^{1} \times\left(\mathbb{P}^{1}\right)^{\mathcal{Z}} \text { for } \mathcal{L}\right) \\
& \text { from Proposition 11.1, } \\
& \subseteq\left\{\mathrm{SL}_{N+1} \text {-stable points of } \operatorname{End}_{d}^{1}[\mathcal{P}] \text { for } \Pi_{\mathcal{P}}^{*} \mathcal{L}\right\} \text { from (47) }
\end{aligned}
$$

This completes the proof of Corollary 11.2. 


\section{The Moduli Space of $\mathbb{P}^{1}$-Endomorphisms with Marked Critical Points}

In characteristic 0 , a degree $d$ map $f: \mathbb{P}^{1} \rightarrow \mathbb{P}^{1}$ has $2 d-2$ geometric critical points, counted with appropriate multiplicity. It is often useful to have a moduli space that classifies maps with marked critical points. Our general construction allows us to describe these spaces as GIT quotients.

Thus suppose that $\operatorname{Crit}(f)=\left\{P_{1}, \ldots, P_{r}\right\}$ consists of $r$ distinct points, and let $e_{i}:=e_{f}\left(P_{i}\right) \geq 2$ denote the multiplicity of $P_{i}$. Then

$$
e_{1}, \ldots, e_{r} \geq 2 \text { and } \sum_{i=1}^{r}\left(e_{i}-1\right)=2 d-2 .
$$

Definition 12.1. Let $\mathcal{P}=\left(\mathcal{V}^{\circ}, \mathcal{V}, \Phi, \epsilon\right)$ be a portrait. We say that $\mathcal{P}$ is a complete critical point portrait of degree $d$ if

$$
\sum_{v \in \mathcal{V}^{\circ}}(\epsilon(v)-1)=2 d-2 \text { and } \mathcal{V}=\bigcup_{v \in \operatorname{Crit}(\mathcal{P})} \mathcal{O}_{\Phi}(v)
$$

(See Definition 3.2 for the definition of $\operatorname{Crit}(\mathcal{P})$ and $\mathcal{O}_{\Phi}(v)$.) We say that $\mathcal{P}$ is critically primitive if it has the further property

$$
\epsilon(v) \geq 2 \text { for all } v \in \mathcal{V}^{\circ} .
$$

We write

$$
\begin{aligned}
\mathrm{P}_{d}^{\text {crit }} & =\{\mathcal{P}: \mathcal{P} \text { is a complete critical point portrait of degree } d\}, \\
\mathrm{P}_{d}^{\text {prim }} & =\left\{\mathcal{P} \in \mathrm{P}_{d}^{\text {crit }}: \mathcal{P} \text { is critically primitive }\right\} .
\end{aligned}
$$

Remark 12.2. See [2, 52 for a description of the set of preperiodic portraits in $\mathrm{P}_{d}^{\text {crit }}$ that are represented by a polynomial map $f(x) \in \bar{K}[x]$, or equivalently, preperiodic portraits $\mathcal{P} \in \mathrm{P}_{d}^{\text {crit }}$ such that $\mathcal{P}$ contains a fixed point of multiplicity $d$ and $\operatorname{End}_{d}^{1}[\mathcal{P}] \neq \emptyset$.

Lemma 12.3. Let $d \geq 2$.

(a) Let $\mathcal{P}=\left(\mathcal{V}^{\circ}, \mathcal{V}, \Phi, \epsilon\right) \in \mathrm{P}_{d}^{\text {crit }}$. Then every $v \in \mathcal{V}$ has either an inarrow or an out-arrow, i.e., either $v \in \mathcal{V}^{\circ}$ or there is some $v^{\prime} \in \mathcal{V}^{\circ}$ with $\Phi\left(v^{\prime}\right)=v$.

(b) $\mathrm{P}_{d}^{\text {prim }}$ contains only finitely many isomorphism classes of portraits.

(c) Every portrait in $\mathrm{P}_{d}^{\text {crit }}$ contains a unique subportrait in $\mathrm{P}_{d}^{\text {prim }}$, which we called the frame of $\mathcal{P}$ and denote by $\operatorname{Fr}(\mathcal{P})$.

Proof. (a) If $v$ is a critical point, i.e, $\epsilon(v) \geq 2$, then necessarily $v \in \mathcal{V}^{\circ}$, so $v$ has an out-arrow. And if $v$ is not a critical point, then it is in the orbit of a critical point, so it has an in-arrow. 
(b) Let $\mathcal{P}=\left(\mathcal{V}^{\circ}, \mathcal{V}, \Phi, \epsilon\right) \in \mathrm{P}_{d}^{\text {prim }}$ be critically primitive. Then

$$
2 d-2=\sum_{v \in \mathcal{V}^{\circ}}(\epsilon(v)-1) \geq \# \mathcal{V}^{\circ},
$$

since each $\epsilon(v) \geq 2$ by assumption. This bounds $\# \mathcal{V}^{\circ}$, and hence also bounds

Further,

$$
\# \mathcal{V}=\#\left(\mathcal{V}^{\circ} \cup \Phi\left(\mathcal{V}^{\circ}\right)\right) \leq 2 \# \mathcal{V}^{\circ}
$$

$$
\sum_{v \in \mathcal{V}^{\circ}} \epsilon(v)=\sum_{v \in \mathcal{V}^{\circ}}(\epsilon(v)-1)+\# \mathcal{V}^{\circ}=2 d-2+\# \mathcal{V}^{\circ}
$$

shows that the multiplicities $\epsilon(v)$ are also bounded.

(c) Let $\mathcal{P}=\left(\mathcal{V}^{\circ}, \mathcal{V}, \Phi, \epsilon_{\mathcal{V}}\right) \in \mathrm{P}_{d}^{\text {crit }}$. We define a subportrait

$$
\mathcal{P}^{\prime}=\left(\mathcal{W}^{\circ}, \mathcal{W}, \Phi_{\mathcal{W}}, \epsilon_{\mathcal{W}}\right)
$$

of $\mathcal{P}$ by taking

$$
\begin{aligned}
\mathcal{W}^{\circ} & :=\left\{v \in \mathcal{V}^{\circ}: \epsilon(v) \geq 2\right\} \\
\mathcal{W} & :=\mathcal{W}^{\circ} \cup\left\{\Phi(w): w \in \mathcal{W}^{\circ}\right\} \\
\Phi_{\mathcal{W}} & :=\left.\Phi\right|_{\mathcal{W}^{\circ}}, \quad \epsilon_{\mathcal{W}}:=\left.\epsilon_{\mathcal{V}}\right|_{\mathcal{W}^{\circ}} .
\end{aligned}
$$

We have

$$
\sum_{w \in \mathcal{W}^{\circ}}\left(\epsilon_{\mathcal{W}}(w)-1\right)=\sum_{\substack{v \in \mathcal{V}^{\circ} \\ \epsilon(w) \geq 2}}\left(\epsilon_{\mathcal{V}}(v)-1\right)=\sum_{v \in \mathcal{V}^{\circ}}\left(\epsilon_{\mathcal{V}}(v)-1\right)=2 d-2
$$

so $\mathcal{P}^{\prime}$ is a complete critical point portrait, and the definitions of $\mathcal{W}$ and $\mathcal{W}^{\circ}$ show that $\mathcal{P}^{\prime}$ is critically primitive.

Next suppose that $\mathcal{P}^{\prime \prime}=\left(\mathcal{U}^{\circ}, \mathcal{U}, \epsilon_{\mathcal{U}}\right) \in \mathrm{P}_{d}^{\text {prim }}$ is a subportrait of $\mathcal{P}$. The assumption that $\left(\mathcal{U}^{\circ}, \mathcal{U}, \epsilon_{\mathcal{U}}\right)$ is critically primitive tells us that $\epsilon_{\mathcal{U}}(u) \geq 2$ for all $u \in \mathcal{U}^{\circ}$, and hence $\mathcal{U}^{\circ} \subseteq \mathcal{W}^{\circ}$ from the definition of $\mathcal{W}^{\circ}$. This allows us to compute

$$
\begin{aligned}
\sum_{u \in \mathcal{U}^{\circ}} & \overbrace{\left(\epsilon_{\mathcal{V}}(u)-\epsilon_{\mathcal{U}}(u)\right)}^{\geq 0 \text { since } \mathcal{P}^{\prime \prime} \subseteq \mathcal{P}}+\sum_{w \in \mathcal{W}^{\circ} \backslash \mathcal{U}^{\circ}} \overbrace{\left(\epsilon_{\mathcal{W}}(w)-1\right)}^{\geq 1 \text { since } \epsilon_{\mathcal{W}}(w) \geq 2 \text { for } w \in \mathcal{W}^{\circ}} \\
& =\sum_{u \in \mathcal{U}^{\circ}}\left(\epsilon_{\mathcal{W}}(u)-\epsilon_{\mathcal{U}}(u)\right)+\sum_{w \in \mathcal{W}^{\circ} \backslash \mathcal{U}^{\circ}}\left(\epsilon_{\mathcal{W}}(w)-1\right) \quad \text { since } \epsilon_{\mathcal{W}}=\epsilon_{\mathcal{V}} \\
& =\sum_{u \in \mathcal{U}^{\circ}}\left(\epsilon_{\mathcal{W}}(u)-1\right)-\sum_{u \in \mathcal{U}^{\circ}}\left(\epsilon_{\mathcal{U}}(u)-1\right)+\sum_{w \in \mathcal{W}^{\circ} \backslash \mathcal{U}^{\circ}}\left(\epsilon_{\mathcal{W}}(w)-1\right) \\
& =\sum_{u \in \mathcal{W}^{\circ}}\left(\epsilon_{\mathcal{W}}(u)-1\right)-\sum_{u \in \mathcal{U}^{\circ}}\left(\epsilon_{\mathcal{U}}(u)-1\right) \\
& =(2 d-2)-(2 d-2) \text { since } \mathcal{W} \text { and } \mathcal{U} \text { are complete }
\end{aligned}
$$




$$
=0 \text {. }
$$

Hence

$$
\mathcal{U}^{\circ}=\mathcal{W}^{\circ} \quad \text { and } \quad \epsilon_{\mathcal{U}}(u)=\epsilon_{\mathcal{V}}(u)=\epsilon_{\mathcal{W}}(u) \text { for all } u \in \mathcal{U}^{\circ} .
$$

Further, we see that

$$
\mathcal{U}=\mathcal{U}^{\circ} \cup \Phi\left(\mathcal{U}^{\circ}\right)=\mathcal{W}^{\circ} \cup \Phi\left(\mathcal{W}^{\circ}\right)=\mathcal{W}
$$

where for the first and third equalities we use (a). This completes the proof that $\mathcal{P}^{\prime \prime}=\mathcal{P}^{\prime}$, and hence that $\mathcal{P}^{\prime}$ is the unique primitive complete critical point subportrait of $\mathcal{P}$.

Definition 12.4. Let $\mathcal{P} \in \mathrm{P}_{d}^{\text {crit }}$, and let $\operatorname{Fr}(\mathcal{P}) \in \mathrm{P}_{d}^{\text {prim }}$ be the frame of $\mathcal{P}$ as described in Lemma 12.3. A critical decomposition series for $\mathcal{P}$ is a sequence of portraits

$$
\mathcal{P}_{0} \subsetneq \mathcal{P}_{1} \subsetneq \cdots \subsetneq \mathcal{P}_{n}
$$

with the portraits $\mathcal{P}_{i}=\left(\mathcal{V}_{i}^{\circ}, \mathcal{V}_{i}, \Phi_{i}, \epsilon_{i}\right) \in \mathrm{P}_{d}^{\text {crit }}$ satisfying

$$
\mathcal{P}_{0}=\operatorname{Fr}(\mathcal{P}), \quad \mathcal{P}_{n}=\mathcal{P}, \quad \text { and } \quad \#\left(\mathcal{V}_{i+1} \backslash \mathcal{V}_{i}\right)=0 \text { or } 1
$$

Remark 12.5. The intuition for adjacent portraits in a critical decomposition series (52) is that $\mathcal{P}_{i+1}$ has exactly one more out-arrow than $\mathcal{P}_{i}$. This can happen in one of three ways:

- Adjoin an existing point $v \in \mathcal{V}_{i} \backslash \mathcal{V}_{i}^{\circ}$ to $\mathcal{V}_{i+1}^{\circ}$ and have $\Phi_{i+1}$ map $v$ to a point in $\mathcal{V}_{i+1}=\mathcal{V}_{i}$.

- Create a new point $v^{\prime}$ in $\mathcal{V}_{i+1}^{\circ}$ and have $\Phi_{i+1}$ map $v^{\prime}$ to a point in $\mathcal{V}_{i}$.

- Create a new point $v^{\prime}$ in $\mathcal{V}_{i+1}$, adjoin an existing point $v \in$ $\mathcal{V}_{i} \backslash \mathcal{V}_{i}^{\circ}$ to $\mathcal{V}_{i+1}^{\circ}$, and have $\Phi_{i+1}$ map $v$ to $v^{\prime}$.

Theorem 10.1(c) tells us that the chain of inclusions in a critical decomposition series (52) gives a chain of inclusions of portrait moduli spaces in the opposite direction,

$$
\mathcal{M}_{d}^{1}\left[\mathcal{P}_{n}\right] \subseteq \cdots \subseteq \mathcal{M}_{d}^{1}\left[\mathcal{P}_{1}\right] \subseteq \mathcal{M}_{d}^{1}\left[\mathcal{P}_{0}\right]
$$

i.e., inclusions that start at $\mathcal{M}_{d}^{1}[\mathcal{P}]$ and end at $\mathcal{M}_{d}^{1}[\operatorname{Fr}(\mathcal{P})]$. We note that $\mathcal{M}_{d}^{1}[\operatorname{Fr}(\mathcal{P})]$ is the largest portrait moduli space using a portrait from $\mathrm{P}_{d}^{\text {crit }}$ in which $\mathcal{M}_{d}^{1}[\mathcal{P}]$ naturally sits. Each inclusion $\mathcal{M}_{d}^{1}\left[\mathcal{P}_{i+1}\right] \subseteq$ $\mathcal{M}_{d}^{1}\left[\mathcal{P}_{i}\right]$ imposes at most one additional critical point relation on the portrait. 


\section{EXAMPLES OF $\mathcal{M}_{d}^{1}[\mathcal{P}]$}

In this section we illustrate our general theory by describing various instances of $\mathcal{M}_{d}^{1}[\mathcal{P}]$ and $\mathcal{M}_{d}^{1}[\mathcal{P} \mid \mathcal{A}]$.

Example 13.1 (Dynatomic Curves). One of the most studied families of dynamical systems is the set of "unicritical" polynomial maps $f_{d, c}(x):=x^{d}+c$, especially for $d=2$. (We have put quote marks around "unicritical" because as a map of $\mathbb{P}^{1}$, the polynomial $f_{d, c}(x)$ has two critical points, 0 and $\infty$.) We briefly explain how these curves fit into our general setup.

For each $d \geq 2$ and $n \geq 1$, there are associated dynatomic curves $Y_{1, d}^{\mathrm{dyn}}(n)$ and $Y_{0, d}^{\mathrm{dyn}}(n)$. The former is frequently defined to be the affine curve consisting of the points $(c, x)$ in $\mathbb{A}^{2}$ satisfying the equation

$$
\prod_{m \mid n}\left(f_{d, c}^{n}(x)-x\right)^{\mu(n / m)}=0 ;
$$

see for example [18]. However, for $d \geq 3$, we note that this is not quite a moduli space, since for every $(d-1)$ st root of unity $\zeta$, we can conjugate $f_{d, c}(x)$ by $x \rightarrow \zeta^{-1} x$, and thus we should identify $(c, x)$ and $(\zeta c, \zeta x)$. So we define $Y_{1, d}^{\text {dyn }}(n)$ to be the quotient of the curve (53) by the action of $(d-1)$ st roots of unity. Of course, for the much studied case of $d=2$, there is no distinction.

The curve $Y_{0, d}^{\text {dyn }}(n)$ classifies degree $d$ unicritical polynomials with a marked cycle of formal period $n$. It is defined to be the quotient

$$
Y_{0, d}^{\mathrm{dyn}}(n):=Y_{1, d}^{\mathrm{dyn}}(n) / /(\mathbb{Z} / n \mathbb{Z}),
$$

where the action of $\mathbb{Z} / n \mathbb{Z}$ is given by

$$
\mathbb{Z} / n \mathbb{Z} \longleftrightarrow \operatorname{Aut}\left(Y_{1, d}^{\mathrm{dyn}}(n)\right), \quad k \longmapsto\left((c, x) \mapsto\left(c, f_{d, c}^{k}(x)\right)\right) .
$$

To describe the dynatomic curve $Y_{1, d}^{\mathrm{dyn}}(n)$ in our general setting, we consider the following portrait $\mathcal{P}=\left(\mathcal{V}^{\circ}, \mathcal{V}, \Phi, \epsilon\right)$ :

$$
\begin{array}{rlrlrl}
\mathcal{V}^{\circ} & =\left\{\alpha, \beta_{1}, \gamma_{0}, \ldots, \gamma_{n-1}\right\}, & \mathcal{V} & =\mathcal{V}^{\circ} \cup\left\{\beta_{2}\right\}, \\
\Phi(\alpha) & =\alpha, & \Phi\left(\beta_{1}\right)=\beta_{2}, & \Phi\left(\gamma_{i}\right) & =\gamma_{i+1 \bmod n,} \\
\epsilon(\alpha) & =d, & \epsilon\left(\beta_{1}\right)=d, & \epsilon\left(\gamma_{0}\right) & =\cdots=\epsilon\left(\gamma_{n-1}\right)=1 .
\end{array}
$$

Let $(f, P) \in \operatorname{End}_{d}^{1}[\mathcal{P}]$ be a geometric point of characteristic 0 . Then $f$ has a maximally ramified critical fixed point, hence is a polynomial, a second maximally ramified critical point, hence is unicritical, and a marked periodic cycle of exact period $n$. Changing coordinates, i.e., conjugating by an element of $\mathrm{PGL}_{2}$, we may assume that $(f, P)$ has the form $\left(f_{d, c}, P\right)$, where $c$ is well-defined up to a $(d-1)$ st root of unity. 
In this way we obtain a well-defined morphism $\mathcal{M}_{d}^{1}[\mathcal{P}] \hookrightarrow Y_{1, d}^{\text {dyn }}(n)$, but the map is not surjective. The failure of surjectivity is due to the fact that we have required the points in our model to be distinct: First, the space $\mathcal{M}_{d}^{1}[\mathcal{P}]$ is missing the points of formal period $n$. Second, it is missing the points where the second critical point is either in the $n$ cycle or maps into the $n$-cycle after one step. Finally, $\mathcal{M}_{d}^{1}[\mathcal{P}]$ is missing the points where the second critical point is fixed. (Note that in the second and third cases $f$ is PCF.) These difficulties are overcome by using instead the space $\overline{\mathcal{M}}_{d}^{1}[\mathcal{P}]$, which is finite over $\mathcal{M}_{d}^{1}$. In this way, we obtain an isomorphism $\overline{\mathcal{M}}_{d}^{1}[\mathcal{P}] \stackrel{\sim}{\rightarrow} Y_{1, d}^{\text {dyn }}(n)$. Similarly, if we let $k \in$ $\mathbb{Z} / n \mathbb{Z}$ act on $\mathcal{P}$ via $\gamma_{i} \mapsto \gamma_{i+k} \bmod n$, then we obtain an isomorphism $\overline{\mathcal{M}}_{d}^{1}[\mathcal{P} \mid \mathbb{Z} / n \mathbb{Z}] \stackrel{\sim}{\rightarrow} Y_{0, d}^{\text {dyn }}(n)$; cf. the notation set in Definition 10.5 .

Example 13.2. The space $\mathcal{M}_{2}^{1}$ has dimension 2, and indeed is isomorphic to $\mathbb{A}^{2}$ via the Milnor isomorphism

$$
\boldsymbol{s}=\left(s_{1}, s_{2}\right): \mathcal{M}_{2}^{1} \stackrel{\sim}{\longrightarrow} \mathbb{A}^{2},
$$

where $s_{1}$ and $s_{2}$ are elementary symmetric functions in the multipliers of the fixed points for the equivalence class of maps $\langle f\rangle \in \mathcal{M}_{2}^{1}$; see [58, Theorem 4.56], for example. The next result, which is due to Blanc, Canci, and Elkies, describes the finite coverings of $\mathcal{M}_{2}^{1}$ associated to portraits consisting of a single cycle of small size.

Theorem 13.3 (Blanc, Canci, and Elkies [3]). Let $\mathcal{C}_{n}$ be the unweighted portrait consisting of a single $n$-cycle, i.e., $\mathcal{V}^{\circ}=\mathcal{V}=\mathbb{Z} / n \mathbb{Z}$ and $\Phi(v)=$ $v+1 \bmod n$. Note that $\operatorname{Aut}\left(\mathcal{C}_{n}\right)$ is the group generated by $\Phi 17$

(a) For $1 \leq n \leq 5$, the variety $\mathcal{M}_{2}^{1}\left[\mathcal{C}_{n} \mid 1\right]$ is a rational surface over $\mathbb{Q}$.

(b) For $1 \leq n \leq 6$, the variety $\mathcal{M}_{2}^{1}\left[\mathcal{C}_{n} \mid 0\right]$ is a rational surface over $\mathbb{Q}$.

(c) The variety $\mathcal{M}_{2}^{1}\left[\mathcal{C}_{6} \mid 1\right]$ is a surface of general type. Thus the Bombieri-Lang conjecture implies that $\mathcal{M}_{2}^{1}\left[\mathcal{C}_{6} \mid 1\right](\mathbb{Q})$ is not Zariski dense.

(d) The set $\mathcal{M}_{2}^{1}\left[\mathcal{C}_{6} \mid 1\right](\mathbb{Q})$ is infinite. More precisely, the space $\mathcal{M}_{2}^{1}\left[\mathcal{C}_{6} \mid 1\right]$ contains a rational curve and three elliptic curves, each of which has infinitely many $\mathbb{Q}$-rational points.

(e) Let $\mathcal{C}_{n}^{\prime}$ denote the union of $\mathcal{C}_{n}$ and a single fixed point. Then the set $\mathcal{M}_{2}^{1}\left[\mathcal{C}_{6}^{\prime} \mid 1\right](\mathbb{Q})$ is infinite.

Example 13.4. Let $\mathcal{P}$ be the portrait consisting of three fixed points,

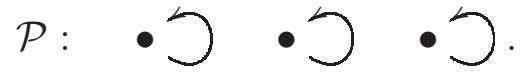

(This is the portrait labeled $\mathcal{P}_{3,6}$ in [56, Table 2].) Every rational map of degree 2 on $\mathbb{P}^{1}$ has three fixed points, counted with multiplicities, so

\footnotetext{
${ }^{17}$ Note that $\operatorname{Aut}\left(\mathcal{C}_{n}\right)$ is not the dihedral group, because our $n$-gon is a directed graph, and automorphisms need to preserve the orientation.
} 
the projection map

$$
\operatorname{End}_{2}^{1}[\mathcal{P}] \rightarrow \operatorname{End}_{2}^{1}
$$

is generically 6 -to-1, corresponding to the six ways to label the three fixed points. However, since $\operatorname{End}_{2}^{1}[\mathcal{P}]$ is, by definition, a subscheme of $\operatorname{End}_{2}^{1} \times\left(\mathbb{P}^{1}\right)_{\Delta}^{3}$, the three specified points are required to be distinct. It follows that the map (54) is étale, and the image is a Zariski open subset of $\operatorname{End}_{2}^{1}$, namely

$$
\operatorname{Image}\left(\operatorname{End}_{2}^{1}[\mathcal{P}]\right)=\left\{f \in \operatorname{End}_{2}^{1}: f \text { has } 3 \text { distinct fixed points }\right\} .
$$

Taking the (partial) closure $\overline{\operatorname{End}}_{2}^{1}[\mathcal{P}]$ fills in the missing maps. Note that there is no issue with formal versus exact period here, since we are only dealing with fixed points.

All of this respects the natural action of $\mathrm{PGL}_{2}$, and we note that $\operatorname{Aut}(\mathcal{P}) \cong \mathcal{S}_{3}$ permutes the three fixed points in $\mathcal{P}$, so we obtain

$$
\begin{array}{ccc}
\mathcal{M}_{2}^{1}[\mathcal{P}] & \underset{\text { étale }}{6 \text {-to-1 }} & \mathcal{M}_{2}^{1}\left[\mathcal{P} \mid \mathcal{S}_{3}\right] \\
\bigcap_{\mathcal{M}_{2}^{1}[\mathcal{P}]} \longrightarrow & \bigcap_{1}^{1} \cong \mathbb{A}^{2} .
\end{array}
$$

The complement $\mathcal{M}_{2}^{1} \backslash \mathcal{M}_{2}^{1}\left[\mathcal{P} \mid \mathcal{S}_{3}\right]$ corresponds to maps having 1 or 2 fixed points. Up to $\mathrm{PGL}_{2}$-conjugation, the only map with 1 fixed point is $z+z^{-1}$, and the maps with 2 fixed points comprise the family ${ }^{18}$

$$
\left\{\left(z^{2}+b z\right) /(z+1): b \neq 1\right\} .
$$

Using the Milnor isomorphism,

$$
\boldsymbol{s}\left(z+z^{-1}\right)=(3,3) \text { and } \boldsymbol{s}\left(\frac{z^{2}+b z}{z+1}\right)=(b+2,2 b+1),
$$

so we see that

$$
\mathcal{M}_{2}^{1}\left[\mathcal{P} \mid \mathcal{S}_{3}\right] \cong \mathbb{A}^{2} \backslash\left\{2 s_{1}=s_{2}+3\right\}
$$

In general, given a map $f$ with three distinct marked fixed points, we can use $\mathrm{PGL}_{2}$ to move the fixed points to $\{0,1, \infty\}$. Then $f$ has the form $f(z)=\left(a z^{2}+b z\right) /(c z+d)$ with $a+b=c+d$. Dehomogenizing $a=$

\footnotetext{
${ }^{18}$ Move the fixed points to 0 and $\infty$, with $\infty$ a double fixed point. The maps that fix 0 and $\infty$ are the maps $f(z)=\left(a z^{2}+b z\right) /(c z+d)$, and $\infty$ is a double fixed point if and only if $a=c$. So setting $a=c$ and dehomogenizing $a=1$, we have $f(z)=$ $\left(z^{2}+b z\right) /(z+d)$. Conjugating by $\varphi(z)=d z$ yields $(1 / d) f(d z)=\left(z^{2}+b z / d\right) /(z+1)$, so relabeling yields the stated result.
} 
1 , and then for convenience letting $b=u-1$ and $d=v$, so $c=u-v$, this puts $f$ into the normal form

$$
f(z)=\frac{z^{2}+(u-1) z}{(u-v) z+v}
$$

with resultant $\operatorname{Res}(f)=u v(1-u+v)$ and with Milnor coordinates

$$
\begin{aligned}
s_{1}(f) & =\frac{(u-1)(v+1)(u-v)}{u v}+2, \\
s_{2}(f) & =\frac{(u-v)\left(u^{2}+v^{2}-u+v+1\right)-1}{u v}+1 \\
& =\frac{(u-1)\left(u^{2}+v^{2}-u v+v+1\right)-v^{3}}{u v}+1 \\
& =\frac{-(v+1)\left(u^{2}+v^{2}-u v-u+1\right)+u^{3}}{u v}+1 .
\end{aligned}
$$

We observe that the excluded locus $2 s_{1}-s_{2}-3=0$ pulls back to

$$
2 s_{1}-s_{2}-3=\frac{(1-u+v)^{3}}{u v} \text {. }
$$

Hence

$$
\mathcal{M}_{2}^{1}[\mathcal{P}] \cong \mathbb{A}^{2} \backslash\{u v(1-u+v)=0\},
$$

where $u v(1-u+v)=0$ corresponds to maps of degree strictly less than 2 .

To illustrate the 6-to-1 nature of the map (55), we observe that the Milnor map sends each of the lines $\{u=1\},\{v=-1\}$, and $\{u=v\}$ in $(u, v)$-space as a double cover onto the line $s_{1}=2$, which is the locus of polynomial maps in $\mathcal{M}_{2}^{1}$. Explicitly,

$u=1 \Rightarrow s_{2}=1-v^{2}, \quad v=-1 \Rightarrow s_{2}=1-u^{2}, \quad u=v \Rightarrow s_{2}=1-u^{-2}$.

The lines $\{u=1\}$ and $\{v=1\}$ map 2-to-1 onto the line $s_{1}=2$. The line $\{u=v\}$ also maps 2-to- 1 to the line $s_{1}=2$, but its image misses the point $\left(s_{1}, s_{2}\right)=(2,1)$.

Example 13.5. Let $\mathcal{P}$ be the portrait consisting of two fixed points and one 2-cycle,

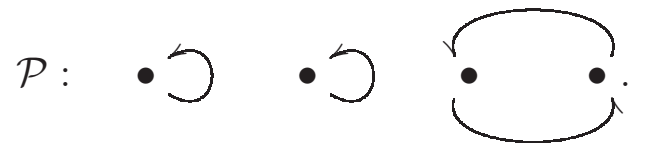

(This is the portrait labeled $\mathcal{P}_{4,13}$ in [56, Table 3].) A generic rational map of degree 2 on $\mathbb{P}^{1}$ has three fixed points and one cycle of period 2 . 
There are 6 ways to choose a labeled pair of fixed points and 2 ways to label the 2-cycle, so the projection map

$$
\operatorname{End}_{2}^{1}[\mathcal{P}] \rightarrow \operatorname{End}_{2}^{1}
$$

is generically 12 -to- 1 .

The automorphism group $\operatorname{Aut}(\mathcal{P}) \cong(\mathbb{Z} / 2 \mathbb{Z})^{2}$ is generated by the involution $\varphi_{1}$ that swaps the fixed points and the involution $\varphi_{2}$ that swaps the two points in the 2-cycle. This gives the commutative diagram of morphisms, where we have indicated the degree of each map:

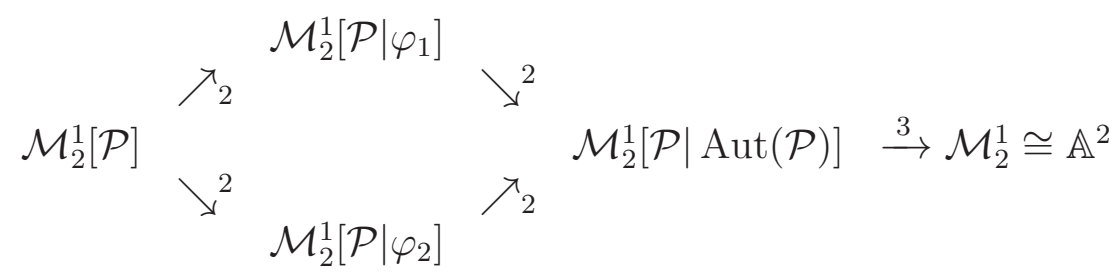

We can use $\mathrm{PGL}_{2}$ to move the points in the 2-cycle to $\{0, \infty\}$ and one of the fixed points to 1 , so $f$ has the form

$$
f(z)=\frac{a z+b}{c z^{2}+d z} \quad \text { with } a+b=c+d .
$$

The fact that $\operatorname{deg}(f)=2$ tells us that $c \neq 0$, so we can dehomogenize $c=1$ to obtain

$$
f(z)=\frac{a z+b}{z^{2}+(a+b-1) z} \quad \text { with } \quad \operatorname{Res}(f)=(1-a) b(a+b) .
$$

The image of $f$ via the Milnor map is given by the unenlightening formulas

$$
\begin{aligned}
& s_{1}(f)=\frac{(b+1) a^{3}+\left(3 b^{2}+b\right) a^{2}+\left(3 b^{3}-2 b^{2}-b\right) a+\left(b^{4}-3 b^{3}-3 b^{2}-b\right)}{-b a^{2}+\left(-b^{2}+b\right) a+b^{2}}, \\
& s_{2}(f)=\frac{-a^{4}-4 b a^{3}+\left(-7 b^{2}+b-1\right) a^{2}+\left(-6 b^{3}+5 b^{2}+b\right) a+\left(-2 b^{4}+6 b^{3}+6 b^{2}+2 b\right)}{-b a^{2}+\left(-b^{2}+b\right) a+b^{2}} .
\end{aligned}
$$

The fixed points of $f$ are the roots of the equation

$$
(z-1)\left(z^{2}+(a+b) z+b\right)=0 .
$$

In order to specify a second fixed point, we need to choose a root of $z^{2}-(a+b) z+b$, which requires taking a square root. Formally, there is a model for $\mathcal{M}_{2}^{1}[\mathcal{P}]$ as an open subvariety of

$$
V:=\left\{(a, b, t) \in \mathbb{A}^{3}:(a+b)^{2}-4 b=t^{2}\right\},
$$

and we have a dominant birational map

$$
\begin{gathered}
V \\
(a, b, t) \longmapsto\left(\frac{a z+b}{z^{2}+(a+b-1) z}, 1, \frac{-a-b+t}{2}, 0, \infty\right),
\end{gathered}
$$


where we have labeled the four points in the picture (56) for $\mathcal{P}$ from left to right.

Example 13.6. We consider maps of degree 3 on $\mathbb{P}^{1}$. Let $\mathcal{P}$ be the portrait consisting of three fixed points, each taken with multiplicity 2 :

$$
\mathcal{P}: \bullet \bigcirc 2 \bullet \bigcup_{2} \bullet \supset_{2}
$$

Without loss of generality, we may move the fixed points to $\{0,1, \infty\}$,

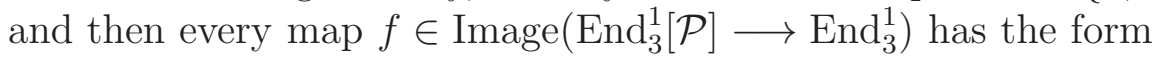

$$
f(z)=\frac{a z^{3}+b z^{2}}{(3 a+2 b) z-(2 a+b)}
$$

after an appropriate change of variables. The resultant of $f$ is

$$
\operatorname{Res}(f)=-2 a^{2}(a+b)^{2}(2 a+b)^{2},
$$

so we find that

$$
\mathcal{M}_{3}^{1}[\mathcal{P}]=\mathbb{P}^{1} \backslash\{[0,1],[1,-1],[1,-2]\} .
$$

Example 13.7. A generic map of degree 3 has 4 fixed points and total ramification index $\sum\left(e_{f}(P)-1\right)=2 d-2=4$, so in principle we could require that all four fixed points be critical points, i.e., we could consider $\mathcal{M}_{3}^{1}\left[\mathcal{P}^{\prime}\right]$ for the portrait

$$
\mathcal{P}^{\prime}: \bullet \bigcirc 2 \quad \bullet \bigcirc_{2} \bullet \bigcirc_{2} \bullet \bigcirc_{2}
$$

This imposes one additional constraint on the 1-parameter family of maps $f(z)$ described by (58), so we might expect examples to exist. However, it turns out that $\mathcal{M}_{3}^{1}\left[\mathcal{P}^{\prime}\right]=\emptyset$, since one easily computes that the fourth fixed point is $2+a^{-1} b$, and the multiplier at that point is

$$
f^{\prime}\left(2+\frac{b}{a}\right)=\frac{3}{2} \text {. }
$$

Hence the fourth fixed point is never critical19 This example illustrates a general way to construct obstructions to $\mathcal{M}_{d}^{1}[\mathcal{P}] \neq \emptyset$ using the fixed point formula described in [58, Theorem 1.14] or [47, Section 12]; see also Section 19.4.

Example 13.8. We investigate a more complicated space $\mathcal{M}_{d}^{1}[\mathcal{P}]$ in which the portrait $\mathcal{P}$ is the union of 3 fixed points and a 3 -cycle containing 2 critical points of multiplicity 2 . We note that the three points in the 3-cycle of $\mathcal{P}$ are rigidly specified, since one is a critical point that is not a critical value, one is a critical value that is not a critical point, and one is both a critical point and a critical value. So

\footnotetext{
${ }^{19}$ More precisely, the fourth fixed point is not critical if $\operatorname{char}(K) \neq 3$, and it is always critical if $\operatorname{char}(K)=3$.
} 
given any $f \in \operatorname{End}_{3}^{1}[\mathcal{P}]$, there is exactly one change of variables taking the 3 -cycle to the points $0,1, \infty$ such that 0 and $\infty$ are critical points and $\infty$ and 1 are critical values. Labeling the three marked fixed points as $\alpha, \beta, \gamma$, we have the picture illustrated in Figure 1.

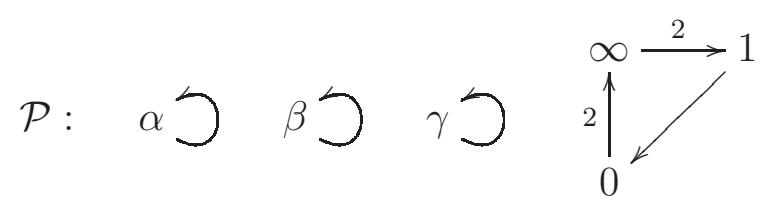

Figure 1. Portrait $\mathcal{P}$ with 3 fixed points and a doublycritical 3-cycle

We first observe that every map $f \in \operatorname{End}_{3}^{1}$ having the 3 -cycle in Figure 1 has the form

$$
f_{u, v}(x)=\frac{x^{3}+u x^{2}-(v+1) x-(u-v)}{x^{3}+u x^{2}}
$$

with

$$
\operatorname{Res}\left(f_{u, v}\right)=-(u+1)(u-v)^{2} v \neq 0 .
$$

Imposing the conditions that $\alpha, \beta, \gamma$ are fixed points of $f_{u, v}$ gives three linear equations for the two quantities $u$ and $v$. Explicitly

$$
\left(\begin{array}{ccc}
\alpha^{3}-\alpha^{2}+1 & \alpha-1 & \alpha\left(\alpha^{3}-\alpha^{2}+1\right) \\
\beta^{3}-\beta^{2}+1 & \beta-1 & \beta\left(\beta^{3}-\beta^{2}+1\right) \\
\gamma^{3}-\gamma^{2}+1 & \gamma-1 & \gamma\left(\gamma^{3}-\gamma^{2}+1\right)
\end{array}\right)\left(\begin{array}{l}
u \\
v \\
1
\end{array}\right)=\left(\begin{array}{l}
0 \\
0 \\
0
\end{array}\right)
$$

We let $A$ denote the matrix in (60) .

This proves that $\left(f_{u, v}, \alpha, \beta, \gamma, 0,1, \infty\right) \in \operatorname{End}_{3}^{1}[\mathcal{P}]$ if and only if the equation (60) has a solution using distinct values $\alpha, \beta, \gamma \notin\{0,1\}$. Clearly a necessary condition for this to occur is to have $\operatorname{det}(A)=0$, and further, if $A$ has rank exactly 2 , then $(\alpha, \beta, \gamma)$ determines a unique pair $(u, v)$.

We pause momentarily to discuss what happens if $\operatorname{rank}(A) \leq 1$. Since we are not allowing $\alpha, \beta, \gamma$ to be 1 and are requiring them to be distinct, a calculation using the 2-by-2 minors of $A$ shows that $\operatorname{rank}(A) \leq 1$ if and only if $\alpha, \beta, \gamma$ are the three roots of the polynomial $t^{3}-t^{2}+1$, in which case $A$ has rank exactly 1 and its null space is the set of vectors of the form $(u, 0, w)$. But the resultant (59) of $f_{u, v}$ vanishes when $v=0$, so these $\alpha, \beta, \gamma$ values do not yield a point in $\operatorname{End}_{3}^{1}[\mathcal{P}] 20$

\footnotetext{
${ }^{20}$ More precisely, we get $\left(1-x^{-2}, \alpha, \beta, \gamma, 0,1 \infty\right) \in \operatorname{End}_{2}^{1}[\mathcal{P}]$.
} 
We conclude that $\mathcal{M}_{3}^{1}[\mathcal{P}]$ is isomorphic to an open subset of the set of $(\alpha, \beta, \gamma) \in \mathbb{A}^{3}$ such that $\operatorname{det}(A)=0$. More precisely, since we want $\alpha, \beta, \gamma$ to be distinct, we take the subvariety of $\mathbb{A}^{3}$ defined by $\frac{\operatorname{det}(A)}{(\alpha-\beta)(\alpha-\gamma)(\beta-\gamma)}=0$. This gives the following formula for a surface $S$ in $\mathbb{A}^{3}$ that contains a Zariski open subset isomorphic to $\mathcal{M}_{3}^{1}[\mathcal{P}]$ :

$$
\begin{gathered}
S: \alpha \beta^{2} \gamma^{2}+\alpha^{2} \beta \gamma^{2}+\alpha^{2} \beta^{2} \gamma-\left(\alpha^{2} \beta^{2}+\alpha^{2} \gamma^{2}+\beta^{2} \gamma^{2}\right) \\
-2\left(\alpha^{2} \beta \gamma+\alpha \beta^{2} \gamma+\alpha \beta \gamma^{2}\right)+3 \alpha \beta \gamma+\alpha^{2} \beta+\alpha^{2} \gamma+\beta^{2} \gamma+\alpha \beta^{2}+\alpha \gamma^{2}+\beta \gamma^{2} \\
\quad-\left(\alpha^{2}+\beta^{2}+\gamma^{2}\right)-2(\alpha \beta+\alpha \gamma+\beta \gamma)+2(\alpha+\beta+\gamma)-1=0 .
\end{gathered}
$$

Next let $\mathcal{A}=\operatorname{Aut}(\mathcal{P}) \cong \mathcal{S}_{3}$ be the set of automorphisms that permute the three fixed points of $\mathcal{P}$. The surface $\mathcal{M}_{3}^{1}[\mathcal{P} \mid \mathcal{A}]$ is obtained by writing the equation of $\mathcal{M}_{3}^{1}[\mathcal{P}]$ in terms of the elementary symmetric functions $X:=\alpha+\beta+\gamma, Y:=\alpha \beta+\alpha \gamma+\beta \gamma$, and $Z:=\alpha \beta \gamma$, which can be done by hand, or using the command IsSymmetric() in Magma [4]. In any case, we find the equation

$$
X^{2}-X Y+Y^{2}-Y Z-2 X+1=0 .
$$

Thus $\mathcal{M}_{3}^{1}[\mathcal{P} \mid \mathcal{A}]$ is a rational surface, since we can solve for $Z$ in terms of $X$ and $Y$. This exhibits $\mathcal{M}_{3}^{1}[\mathcal{P}]$ as a ramified cover of $\mathbb{A}^{2}$.

\section{The Dimension of $\mathcal{M}_{d}^{N}[\mathcal{P}]$ for Unweighted Portraits}

In this section we make the following assumption:

$$
\text { All varieties are defined over a field of characteristic } 0 \text {. }
$$

Definition 14.1. For integers $d \geq 2, N \geq 1$, and $n \geq 1$, we set

$$
\nu_{d}^{N}(n):=\sum_{k \mid n}\left(\mu(n / k) \sum_{j=0}^{N} d^{j k}\right) .
$$

For $m \geq 1$ we set

$$
\nu_{d}^{N}(m, n):=d^{N(m-1)}\left(d^{N}-1\right) \nu_{d}^{N}(n),
$$

and we extend this to $m=0$ by setting $\nu_{d}^{N}(0, n):=\nu_{d}^{N}(n)$.

Theorem 14.2. Let $\mathcal{P}=\left(\mathcal{V}^{\circ}, \mathcal{V}, \Phi\right)$ be an unweighted portrait, and let

$$
\begin{aligned}
D_{\mathcal{P}} & :=\max \left\{\# \Phi^{-1}(P): P \in \mathcal{V}\right\}, \\
C_{\mathcal{P}}(n) & :=\#\left\{v \in \mathcal{V}^{\circ}: v \text { has exact period } n \text { for } \Phi\right\} .
\end{aligned}
$$

Then the following are equivalent:

(a) $\operatorname{End}_{d}^{N}[\mathcal{P}] \neq \emptyset$.

(b) $\mathcal{M}_{d}^{N}[\mathcal{P}] \neq \emptyset$. 
(c) The finite morphism

$$
\Pi_{\mathcal{P}}: \overline{\operatorname{End}}_{d}^{N}[\mathcal{P}] \longrightarrow \operatorname{End}_{d}^{N} \times\left(\mathbb{P}^{N}\right)^{\mathcal{V} \backslash \mathcal{V}^{\circ}}
$$

described in Theorem 9.1(a) is surjective.

(d) $\operatorname{dim} \operatorname{End}_{d}^{N}[\mathcal{P}]=\operatorname{dim} \operatorname{End}_{d}^{N}+N \cdot \#\left(\mathcal{V} \backslash \mathcal{V}^{\circ}\right)$

(e) $\operatorname{dim} \mathcal{M}_{d}^{N}[\mathcal{P}]=\operatorname{dim} \mathcal{M}_{d}^{N}+N \cdot \#\left(\mathcal{V} \backslash \mathcal{V}^{\circ}\right)$.

(f) $D_{\mathcal{P}} \leq d^{N}$ and $C_{\mathcal{P}}(n) \leq \nu_{d}^{N}(n)$ for all $n \geq 1$.

The remainder of this section is devoted to proving Theorem 14.2 and a corollary that describes the dimensions of the fibers and images of maps between portrait moduli spaces.

Definition 14.3. For $f \in \operatorname{End}_{d}^{N}$, we let $\operatorname{PrePer}(f)$ denote the set of preperiodic points for $f$. For each preperiodic point $P \in \operatorname{PrePer}(f)$, we say $P$ has preperiodic type $(m, n)$ if $P$ enters an $n$-cycle after precisely $m$ steps. We will also refer to $m$ and $n$ as the preperiod and (eventual) period, respectively, of $P$.

Lemma 14.4. Fix integers $d \geq 2$ and $N \geq 1$, and let

$$
f: \mathbb{P}_{\operatorname{End}_{d}^{N}}^{N} \longrightarrow \mathbb{P}_{\operatorname{End}_{d}^{N}}^{N}
$$

be the generic endomorphism of degree $d$. Then for all integers $m \geq$ 0 and $n \geq 1$, the map $f$ has exactly $\nu_{d}^{N}(m, n)$ geometric points of preperiodic type $(m, n)$.

Proof. We first consider the case $m=0$. The number of points of period $n$ for $f$, counted with the appropriate multiplicity, is equal to $\nu_{d}^{N}(n)$; see [29, Theorems 4.3 and 4.17]. By [30, page 3513], for a general degree- $d$ endomorphism of $\mathbb{P}^{N}$, each point of exact period $n$ occurs to multiplicity 1 , so there must be exactly $\nu_{d}^{N}(n)=\nu_{d}^{N}(0, n)$ such points.

By [22, Corollary 3.5], the generic map $f$ has no ramified preperiodic points, so every preperiodic point has precisely $d^{N}$ preimages under $f$. Every point of type $(1, n)$ is the preimage of a point of period $n$; conversely, given a point $P$ of period $n$, all but one of its preimages is a point of type $(1, n)$. (The remaining preimage is the periodic point $f^{n-1}(P)$.) Thus $f$ has $\left(d^{N}-1\right) \nu_{d}^{N}(n)=\nu_{d}^{N}(1, n)$ points of type $(1, n)$.

Now assume that $m \geq 2$. The points of type $(m, n)$ are precisely the preimages of the points of type $(m-1, n)$. If we assume that there are $\nu_{d}^{N}(m-1, n)$ points of type $(m-1, n)$, and if we again use the fact that $f$ is unramified at its preperiodic points, it follows that there are exactly

$$
d^{N} \cdot \nu_{d}^{N}(m-1, n)=\nu_{d}^{N}(m, n)
$$


points of type $(m, n)$. Thus the stated result follows by induction on $m$.

Proof of Theorem 14.2. We prove various implications.

$(\mathrm{a}) \Longleftrightarrow(\mathrm{b})$ This is immediate from the surjectivity of the map

$$
\operatorname{End}_{d}^{N}[\mathcal{P}] \rightarrow \mathcal{M}_{d}^{N}[\mathcal{P}]
$$

$(\mathrm{d}) \Longrightarrow(\mathrm{c})$ Theorem 9.1 says that the map (62) is a finite morphism, and (d) says that $\overline{\operatorname{End}}_{d}^{N}[\mathcal{P}]$ contains an open subset (namely, $\operatorname{End}_{d}^{N}[\mathcal{P}]$ ) whose dimension is equal to the dimension of the codomain $\operatorname{End}_{d}^{N} \times\left(\mathbb{P}^{N}\right)^{\mathcal{V} \backslash \mathcal{V}^{\circ}}$. Further, the codomain is irreducible, since $\operatorname{End}_{d}^{N}$ is an open subset of $\mathbb{P}^{M}$. Hence the map (62) is surjective.

$(\mathrm{d}) \Longleftrightarrow(\mathrm{e})$ These implications follow from the fact that the generic fibers of $\operatorname{End}_{d}^{N}[\mathcal{P}] \rightarrow \mathcal{M}_{d}^{N}[\mathcal{P}]$ and $\operatorname{End}_{d}^{N} \rightarrow \mathcal{M}_{d}^{N}$ have dimension equal to the dimension of $\mathrm{PGL}_{N+1}$.

$(\mathrm{c}) \Longrightarrow(\mathrm{a})$ The assumed surjectivity of the map (62) implies in particular that $\overline{\operatorname{End}}_{d}^{N}[\mathcal{P}] \neq \emptyset$. But $\overline{\operatorname{End}}_{d}^{N}[\mathcal{P}]$ is the Zariski closure of $\operatorname{End}_{d}^{N}[\mathcal{P}]$ in $\operatorname{End}_{d}^{N} \times\left(\mathbb{P}^{N}\right)^{\mathcal{V} \backslash \mathcal{V}^{\circ}}$, so $\operatorname{End}_{d}^{N}[\mathcal{P}]$ cannot be the empty set.

$(\mathrm{a}) \Longrightarrow(\mathrm{f}) \quad$ A degree $d$ map $f$ on $\mathbb{P}^{N}$ has topological degree $d^{N}$, and Lemma 14.4 tells us that $f$ can have at most $\nu_{d}^{N}(n)$ points of period $n$. Hence (a) implies (f).

$(\mathrm{f}) \Longrightarrow(\mathrm{a})$ To see the opposite implication, we suppose that the inequalities in (f) are true. Let $\mathcal{V}^{\prime} \subseteq \mathcal{V}$ be the set of preperiodic vertices of $\mathcal{P}$, let $\mathcal{P}^{\prime}=\left(\mathcal{V}^{\prime}, \Phi\right)$ be the preperiodic subportrait of $\mathcal{P}$, and let $m_{0}$ and $n_{0}$ be the maximal preperiod and period, respectively, for a vertex $v \in \mathcal{V}^{\prime}$. We relabel the vertices in $\mathcal{V}$ so that

$\mathcal{V}=\{1, \ldots, s\}, \quad \mathcal{V}^{\circ}=\{q, \ldots, s\}, \quad \mathcal{V}^{\prime}=\{r, \ldots, s\}, \quad$ with $q \leq r \leq s$,

i.e., the vertices are labeled from 1 to $s$ so that

$$
\underbrace{1,2, \ldots, q-1}_{\text {vertices with no out-arrow }}, \underbrace{q, q+1, \ldots, r-1, \overbrace{r, r+1, \ldots, s}^{\text {preperiodic vertices }}}_{\text {vertices having an out-arrow }} .
$$

The fact that the generic degree $d$ map on $\mathbb{P}^{N}$ has no ramified preperiodic points implies that for each $(m, n)$ with $0 \leq m \leq m_{0}$ and $1 \leq n \leq n_{0}$, there is a open subset of $f \in \operatorname{End}_{d}^{N}$ such that the map $f$ has precisely $\nu_{d}^{N}(m, n)$ points of type $(m, n)$. Hence we can find a map $f$ and points $P_{r}, \ldots, P_{s} \in \mathbb{P}^{N}$ so that $\left(f, P_{r}, \ldots, P_{s}\right)$ is a model for $P^{\prime}$. 
We next construct points $P_{k}$ for vertices $k \in \mathcal{V} \backslash \mathcal{V}^{\circ}=\{1, \ldots, q-1\}$ that do not have an out-arrow. For each such $k$, we let

$$
m_{k}:=\max \left\{m: \Phi^{m}(v)=k \text { for some } v \in \mathcal{V}\right\} .
$$

We then successively choose points $P_{q-1}, \ldots, P_{2}, P_{1} \in \mathbb{P}^{N}$ so that

$$
P_{k} \notin \bigcup_{1 \leq m \leq m_{k}} \operatorname{Per}_{m}(f) \cup \bigcup_{m=0}^{m_{k}} \bigcup_{i=k+1}^{s} f^{m}\left(P_{i}\right) \cup \bigcup_{m=0}^{m_{k}} f^{m}(\operatorname{Crit}(f)),
$$

where $\operatorname{Crit}(f)$ is the critical locus (also called the ramification locus) of $f$.

It remains to construct points $P_{q}, \ldots, P_{r-1} \in \mathbb{P}^{N}$ that complete our model for $\mathcal{P}$. These correspond to the vertices $\{q, \ldots, r-1\}$ that have out-arrows, but whose orbits terminate at points with no out-arrow. We do this recursively as follows:

(1) Initialize $\mathcal{L}:=\{q, \ldots, r-1\}$.

(2) Choose a vertex $\ell \in \mathcal{L}$ such that $P_{\Phi(\ell)}$ has already been assigned.

(3) Let $v_{1}, \ldots, v_{t}$ be the vertices in $\Phi^{-1}(\Phi(\ell))$. Note that $t \geq 1$, since $\ell \in \Phi^{-1}(\Phi(\ell))$.

(4) Choose distinct points $P_{v_{1}}, \ldots, P_{v_{t}} \in \mathbb{P}^{N}$ satisfying

$$
f\left(P_{v_{1}}\right)=\cdots=f\left(P_{v_{t}}\right)=P_{\ell} .
$$

(5) Remove $v_{1}, \ldots, v_{t}$ from $\mathcal{L}$. If $\mathcal{L}=\emptyset$ terminate; otherwise go to Step (2) and choose another vertex in $\mathcal{L}$.

We claim that this process yields a model $\left(f, P_{1}, \ldots, P_{s}\right)$ for $\mathcal{P}$. First, we verify that it is in fact possible to choose the $P_{i}$ as we have described. For $1 \leq k<q$, i.e., for vertices with no out-arrows, the set (63) that we need to avoid when choosing $P_{k}$ is a set of codimension 1 in $\mathbb{P}^{N}$, since it consists of a union of a finite set of points together with some forward images of the critical locus, which has codimension 1. Hence these $P_{k}$ may be chosen from a non-empty Zariski open set.

For vertices $\ell$ with an out-arrow whose orbits terminate at a point with no out-arrow, i.e., $q \leq \ell<r$, Step (4) requires that we choose $t$ distinct points in $f^{-1}\left(P_{\ell}\right)$, where $t=\# \Phi^{-1}(\Phi(\ell))$. Our assumption that $\mathcal{D}_{P} \leq d^{N}$ means that $t \leq d^{N}$, while (63) tells us that $P_{\ell} \notin f(\operatorname{Crit}(f))$, i.e., $P_{\ell}$ is not a critical value of $f$, so $\# f^{-1}\left(P_{\ell}\right)=d^{N}$. Hence there are enough distinct points in the inverse image $f^{-1}\left(P_{\ell}\right)$ to accomplish Step (4).

We also note that the algorithm (1)-(5) terminates, since each time we get to Step (5), we remove at least one vertex from the set $\mathcal{L}$.

Finally, it is clear from the construction that for each $v \in \mathcal{V}^{\circ}$, i.e., for each vertex with an out-arrow, we have $f\left(P_{v}\right)=P_{\Phi(v)}$. And the 
points $P_{1}, \ldots, P_{s}$ are distinct due to the restrictions imposed by (63)

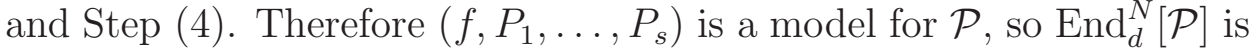
non-empty.

We have now completed the proof of the following implications:

$$
(\mathrm{e}) \Longleftrightarrow(\mathrm{d}) \Longrightarrow(\mathrm{c}) \Longrightarrow(\mathrm{a}) \Longleftrightarrow(\mathrm{b}) \Longleftrightarrow(\mathrm{f})
$$

In order to complete the proof of Theorem 14.2, it suffices to prove that (a) implies (d). We first prove some lemmas describing various codimensions.

Lemma 14.5. Fix integers $d \geq 2$ and $N \geq 1$. For each pair of integers $(m, n)$ with $m \geq 0$ and $n \geq 1$, define $R_{d}^{N}(\bar{m}, n) \subset \operatorname{End}_{d}^{N}$ to be the set of endomorphisms $f$ of $\mathbb{P}^{N}$ with fewer than $\nu_{d}^{N}(m, n)$ points of preperiodic portrait $(m, n)$. Then $R_{d}^{N}(m, n)$ has codimension 1 in $\operatorname{End}_{d}^{N}$.

Proof. First let $m=0$. If $f$ has fewer than $\nu_{d}^{N}(n)$ points of period $n$, then [29, Theorems 2.20 and 3.1] tells us that one of the period- $n$ orbits of $f$ has a multiplier that is an $n$th root of unity.

For each point $\alpha$ of period $n$ for the generic map $f$, write $J_{\alpha}$ for the Jacobian matrix of $f^{n}$ at $\alpha$, and for each $n$th root of unity $\zeta$, let $\lambda_{\alpha, \zeta}$ be the determinant of $J_{\alpha}-\zeta I_{N}$, where $I_{N}$ is the $N \times N$ identity matrix. Let $\lambda$ denote the product of $\lambda_{\alpha, \zeta}$ over all $\nu_{d}^{N}(n)$ points $\alpha$ of period $n$ for $f$ and all $\zeta \in \mu_{n}$. Then $\lambda$ lies in the function field of $\operatorname{End}_{d}^{N}$, since it is symmetric in the periodic points of $f$, so its vanishing will cut out $R_{d}^{N}(0, n)$ as a closed subscheme of $\operatorname{End}_{d}^{N}$ of codimension at most 1 . By Lemma 14.4, however, $R_{d}^{N}(0, n)$ is a proper subscheme of $\operatorname{End}_{d}^{N}$, hence the codimension is equal to 1 .

Now let $m \geq 1$. For each $\beta \in \mathbb{P}_{\text {End }_{d}^{N}}^{N}$, we impose a polynomial condition $\lambda_{\beta}$ by forcing the Jacobian $J_{\beta}$ of $f$ at $\beta$ to have a nontrivial kernel, and we take $\lambda$ to be the product of $\lambda_{\beta}$ over all $\beta$ of eventual period $n$ and preperiod at most $m$. As in the previous paragraph, $\lambda$ cuts out a codimension 1 closed subscheme $S_{d}^{N}(m, n)$ of $\operatorname{End}_{d}^{N}$, and we have

$$
R_{d}^{N}(m, n)=R_{d}^{N}(0, n) \cup S_{d}^{N}(m, n),
$$

which completes the proof of Lemma 14.5.

Lemma 14.6. Let $\mathcal{P}$ be an unweighted portrait such that $\operatorname{End}_{d}^{N}[\mathcal{P}]$ is nonempty, and let

$$
R_{d}^{N}(\mathcal{P}):=\operatorname{End}_{d}^{N} \backslash \operatorname{Image}\left(\operatorname{End}_{d}^{N}[\mathcal{P}] \rightarrow \operatorname{End}_{d}^{N}\right),
$$

i.e., let $R_{d}^{N}(\mathcal{P})$ be the subscheme of $\operatorname{End}_{d}^{N}$ consisting of those maps $f$ that cannot be extended to give a model of $\mathcal{P}$. Then $R_{d}^{N}(\mathcal{P})$ has codimension at least 1 in $\operatorname{End}_{d}^{N}$. 
Proof. If $f \in R_{d}^{N}(\mathcal{P})$, then there exist some integers $m \geq 0$ and $n \geq$ 1 such that $f$ has fewer points of preperiodic type $(m, n)$ than the portrait $\mathcal{P}$, which itself has at most $\nu_{d}^{N}(m, n)$ points of type $(m, n)$ from the implication $(\mathrm{a}) \Longrightarrow(\mathrm{f})$ that we already proved in Theorem 14.2 . Thus, if $n_{0}$ and $m_{0}$ denote the maximal period and preperiod of a preperiodic vertex of $\mathcal{P}$, then

$$
R_{d}^{N}(\mathcal{P}) \subseteq \bigcup_{\substack{0 \leq m \leq m_{0} \\ 1 \leq n \leq n}} R_{d}^{N}(m, n)
$$

and Lemma 14.5 says that each $R_{d}^{N}(m, n)$ has codimension 1 .

Lemma 14.7. Let $\mathcal{P}=\left(\mathcal{V}^{\circ}, \mathcal{V}, \Phi\right)$ be an unweighted portrait. Let

$$
\pi_{\mathcal{P}}: \operatorname{End}_{d}^{N}[\mathcal{P}] \rightarrow \operatorname{End}_{d}^{N}, \quad \pi_{\mathcal{P}}\left(f, P_{1}, \ldots, P_{k}\right)=f
$$

be the natural projection map. Then for every $f \in \operatorname{End}_{d}^{N}$ we have

$$
\operatorname{dim}\left(\pi_{\mathcal{P}}^{-1}(f)\right)= \begin{cases}N \cdot \#\left(\mathcal{V} \backslash \mathcal{V}^{\circ}\right) & \text { if } f \notin R_{d}^{N}(\mathcal{P}), \\ 0 & \text { if } f \in R_{d}^{N}(\mathcal{P})\end{cases}
$$

Proof. We first note that by definition of $R_{d}^{N}(\mathcal{P})$, we have $f \in R_{d}^{N}(\mathcal{P})$ if and only if the fiber $\pi_{\mathcal{P}}^{-1}(f)$ is empty. We may therefore assume that $f \notin R_{d}^{N}(\mathcal{P})$. Let $r:=\#\left(\mathcal{V} \backslash \mathcal{V}^{\circ}\right)$.

First suppose that $\mathcal{V}^{\circ}=\emptyset$, i.e., suppose that $\mathcal{P}$ consists of $r$ vertices with no arrows. Then clearly

$$
\operatorname{End}_{d}^{N}[\mathcal{P}] \cong \operatorname{End}_{d}^{N} \times\left(\mathbb{P}^{N}\right)_{\Delta}^{r}
$$

and the map $\pi_{\mathcal{P}}$ is just projection onto the $\operatorname{End}_{d}^{N}$ factor. Therefore each fiber is isomorphic to $\left(\mathbb{P}^{N}\right)_{\Delta}^{r}$, which is an open subset of $\left(\mathbb{P}^{N}\right)^{r}$, hence has dimension $N r$.

Next suppose $\mathcal{V}^{\circ} \neq \emptyset$. Write

$$
\mathcal{V}=\left\{P_{1}, P_{2}, \ldots, P_{r}, Q_{1}, Q_{2}, \ldots, Q_{s}\right\},
$$

where $\mathcal{V}^{\circ}=\left\{Q_{1}, \ldots, Q_{s}\right\}$. Let $\mathcal{P}^{\prime}$ be the subportrait of $\mathcal{P}$ consisting only of the vertices in $\mathcal{V} \backslash \mathcal{V}^{\circ}$. Thus $\mathcal{V}^{\prime}=\left\{P_{1}, \ldots, P_{r}\right\}$ and $\mathcal{V}^{\prime \circ}=\emptyset$. Then $\pi_{\mathcal{P}}=\pi_{\mathcal{P}^{\prime}} \circ \phi$, where $\phi$ is the map

$$
\begin{aligned}
\phi: \operatorname{End}_{d}^{N}[\mathcal{P}] & \longrightarrow \operatorname{End}_{d}^{N}\left[\mathcal{P}^{\prime}\right] \\
\left(f,\left(x_{1}, \ldots, x_{r}, y_{1}, \ldots, y_{s}\right)\right) & \longmapsto\left(f,\left(x_{1}, \ldots, x_{r}\right)\right) .
\end{aligned}
$$

It remains to show that the fibers of $\phi$ are finite.

Suppose we have fixed the map $f$ and the points $x_{1}, \ldots, x_{r}$. Since $\# \mathcal{V}$ is finite, the orbit of each $y_{i}$ under $f$ must eventually reach either one of the $x_{j}$ or a periodic point. Since there are finitely many points 
with periods specified by $\mathcal{P}$, and each point has at most $d^{N}$ preimages under $f$, we are done with the proof of Lemma 14.7.

We now have the tools required to prove the final implication that completes the proof of Theorem 14.2.

$(\mathrm{a}) \Longrightarrow(\mathrm{d})$ Let $U_{d}^{N}(\mathcal{P})=\operatorname{End}_{d}^{N} \backslash R_{d}^{N}(\mathcal{P})$. By definition, the image of the map $\pi_{\mathcal{P}}$ in Lemma 14.7 is equal to $U_{d}^{N}(\mathcal{P})$. Further, Lemma 14.6 tells us that $U_{d}^{N}(\mathcal{P})$ has full dimension, i.e., that $\operatorname{dim} U_{d}^{N}(\mathcal{P})=\operatorname{dim} \operatorname{End}_{d}^{N}$. The desired result follows from this and the fact that each fiber over $U_{d}^{N}(\mathcal{P})$ has dimension $N \cdot \#\left(\mathcal{V} \backslash \mathcal{V}^{\circ}\right)$.

We conclude this section with a general result describing the dimensions of the fibers and images of maps between portrait moduli spaces.

Theorem 14.8. Let $\mathcal{P}^{\prime} \subseteq \mathcal{P}$ be an inclusion of unweighted portraits, let

$$
M_{\mathcal{P}, \mathcal{P}^{\prime}}: \mathcal{M}_{d}^{N}[\mathcal{P}] \longrightarrow \mathcal{M}_{d}^{N}\left[\mathcal{P}^{\prime}\right]
$$

be the natural induced map on moduli spaces as described in Theorem [10.1, and assume that $\mathcal{M}_{d}^{N}[\mathcal{P}]$ is non-empty. Viewing $\mathcal{P}$ as a directed graph, let $n$ be the number of connected components of $\mathcal{P}$ that intersect $\mathcal{P}^{\prime}$ and do not contain a cycle. Then

$$
\begin{aligned}
\operatorname{dim}\left(\text { Fiber of } M_{\mathcal{P}, \mathcal{P}^{\prime}}\right) & =N \cdot\left(\#\left(\mathcal{V} \backslash \mathcal{V}^{\circ}\right)-n\right), \\
\operatorname{codim}\left(\text { Image of } M_{\mathcal{P}, \mathcal{P}^{\prime}}\right) & =N \cdot\left(\#\left(\mathcal{V}^{\prime} \backslash \mathcal{V}^{\prime \circ}\right)-n\right) .
\end{aligned}
$$

Proof. Let $\left\langle f,\left(P_{v^{\prime}}\right)_{v^{\prime} \in \mathcal{V}^{\prime}}\right\rangle \in \mathcal{M}_{d}^{N}\left[\mathcal{P}^{\prime}\right]$ be in the image of $M_{\mathcal{P}, \mathcal{P}^{\prime}}$, and let $\left\langle f,\left(P_{v}\right)_{v \in \mathcal{V}}\right\rangle \in \mathcal{M}_{d}^{N}[\mathcal{P}]$ be a preimage. Much as in the proof of Lemma 14.7, a point $P_{v} \in \mathbb{P}^{N}$ corresponding to the vertex $v \in \mathcal{V}$ may be chosen freely from a Zariski open subset of $\mathbb{P}^{N}$ if and only if $v$ satisfies both of the following conditions:

- $v$ has no outgoing arrow, i.e., $v \notin \mathcal{V}^{\circ}$.

- $v$ is not in a component of $\mathcal{P}$ containing vertices from $\mathcal{P}^{\prime}$, since otherwise there would be an $f$-orbit relation between $P_{v}$ and a point $P_{v^{\prime}}$ with $v^{\prime} \in \mathcal{V}^{\prime}$.

Hence (65) is true.

Next, we know from the $(\mathrm{a}) \Longrightarrow(\mathrm{e})$ implication of Theorem 14.2 that

$$
\operatorname{dim} \mathcal{M}_{d}^{N}[\mathcal{P}]=\operatorname{dim} \mathcal{M}_{d}^{N}+N \cdot \#\left(\mathcal{V} \backslash \mathcal{V}^{\circ}\right)
$$

Using this, we can compute the dimension of the image of $M_{\mathcal{P}, \mathcal{P}^{\prime}}$ as follows:

$$
\begin{aligned}
\operatorname{dim}\left(\text { Image of } M_{\mathcal{P}, \mathcal{P}^{\prime}}\right) \\
=\operatorname{dim} \mathcal{M}_{d}^{N}[\mathcal{P}]-\operatorname{dim}\left(\text { Fiber of } M_{\mathcal{P}, \mathcal{P}^{\prime}}\right)
\end{aligned}
$$




$$
\begin{aligned}
& =\left(\operatorname{dim} \mathcal{M}_{d}^{N}+N \cdot \#\left(\mathcal{V} \backslash \mathcal{V}^{\circ}\right)\right)-N \cdot\left(\#\left(\mathcal{V} \backslash \mathcal{V}^{\circ}\right)-n\right) \\
& =\operatorname{dim} \mathcal{M}_{d}^{N}+N \cdot n
\end{aligned}
$$

Subtracting this from the formula for $\operatorname{dim} \mathcal{M}_{d}^{N}\left[\mathcal{P}^{\prime}\right]$ in Theorem 14.2(e) gives the desired value for the codimension of the image of $M_{\mathcal{P}, \mathcal{P}^{\prime}}$.

\section{The Dimension of $\mathcal{M}_{d}^{1}[\mathcal{P}]$ for Weighted Portraits}

In this section we study $\mathcal{M}_{d}^{1}[\mathcal{P}]$, where $\mathcal{P}$ is now allowed to be a weighted portrait. We assume throughout that we are working over a field of characteristic 0. As noted in Remark 7.5, there are geometric constraints that may force $\mathcal{M}_{d}^{N}[\mathcal{P}]=\emptyset$. For example, if $\mathcal{P}$ contains four fixed points, or if $\mathcal{P}$ contains two periodic cycles of length 2 , then $\mathcal{M}_{2}^{1}[\mathcal{P}]=\emptyset$; see Theorem $14.2(\mathrm{f})$. More generally, we have the following natural geometric conditions that are necessary, but may not be sufficient, to ensure that $\mathcal{M}_{d}^{1}[\mathcal{P}]$ is non-empty.

Proposition 15.1. Let $d \geq 2$, and let $\mathcal{P}=\left(\mathcal{V}^{\circ}, \mathcal{V}, \Phi, \epsilon\right)$ be a portrait such that $\mathcal{M}_{d}^{1}[\mathcal{P}] \neq \emptyset$. Then $\mathcal{P}$ satisfies the following conditions:

$$
\text { (I) } \max _{v \in \mathcal{V}} \sum_{w \in \Phi^{-1}(v)} \epsilon(w) \leq d . \quad \text { (II) } \quad \sum_{w \in \mathcal{V}^{\circ}}(\epsilon(w)-1) \leq 2 d-2 .
$$

For all $n \geq 1$,

$$
\left(\mathrm{III}_{n}\right) \quad \#\left\{w \in \mathcal{V}^{\circ}: \begin{array}{l}
\Phi^{n}(w)=w \text { and } \\
\Phi^{m}(w) \neq w \text { for all } m<n
\end{array}\right\} \leq \nu_{d}^{1}(n) .
$$

(See (61) in Definition 14.1 for the definition of $\nu_{d}^{N}(n)$.)

Proof. Constraint I comes from the fact that $f$ is a map of degree $d$, so a point has at most $d$ preimages. Constraint II follows from the Riemann-Hurwitz formula $\sum\left(e_{f}(P)-1\right)=2 d-2$; see [58, Theorem 1.1]. Constraint $\mathrm{III}_{n}$ is true because a degree $d$ map on $\mathbb{P}^{1}$ has at most the indicated number of points of exact period $n$; see [58, Remark 4.3]. We remark that if $\mathcal{P}$ is unweighted, then constraint (II) is vacuous.

Remark 15.2. For unweighted portraits, Theorem 14.2(f) gives succinct necessary and sufficient condition for $\mathcal{M}_{d}^{1}[\mathcal{P}]$ to be nonempty. The situation is more complicated in the case of weighted portraits. For example, if $\mathcal{P}$ consists of four fixed points, each having multiplicity 2 , then the explicit computation in Example 13.7 shows that $\mathcal{M}_{3}^{1}[\mathcal{P}]=\emptyset$, despite the fact that $\mathcal{P}$ satisfies properties (I), (II), and $\left(\mathrm{III}_{n}\right)$ for all $n \geq 1$ with $d=3$ in Proposition 15.1. 
Remark 15.3. As illustrated in Remark 14.2, the elementary conditions given in Proposition 15.1 for a portrait to be realized by a degree $d$ rational map are necessary but not sufficient. A deeper topological characterization of rational functions was formulated by Thurston and proved by Douady and Hubbard in [15]. We give a brief description. Let $F: S^{2} \rightarrow S^{2}$ be a topological branched cover of the 2-sphere, and suppose that the forward orbits of the branch points are finite. Thurston defines $F$ to be equivalent to a $\mathrm{PCF}$ rational map $f: \mathbb{P}^{1}(\mathbb{C}) \rightarrow \mathbb{P}^{1}(\mathbb{C})$ if there is a homeomorphism of $S^{2} \cong \mathbb{P}^{1}(\mathbb{C})$ taking $F$ to $f$ and correctly identifying the postcritical set $P_{F}$ of $F$ with the postcritical set $P_{f}$ of $f$. Thurston uses $F$ and $P_{F}$ to define a map $\sigma_{F}: \operatorname{Teich}\left(S^{2}, P_{F}\right) \rightarrow \operatorname{Teich}\left(S^{2}, P_{F}\right)$ on a certain Teichmüller space. Then his topological characterization says that $F$ is equivalent to a PCF rational map if and only if the Thurston pullback map $\sigma_{F}$ has a fixed point.

Remark 15.4. As noted earlier, in this section we work over a field of characteristic 0 . To see why, we note that otherwise there may be problems with Condition (II) in Proposition 15.1. For example, let $\mathcal{P}$ consist of 3 fixed points, each of multiplicity 2 . Then $\mathcal{M}_{2}^{1}[\mathcal{P}](\mathbb{C})=\emptyset$, as specified by Proposition 15.1. But $\mathcal{M}_{2}^{1}[\mathcal{P}]\left(\mathbb{F}_{2}\right)$ contains a point. Indeed,

$$
\mathcal{M}_{2}^{1}[\mathcal{P}]\left(\mathbb{F}_{2}\right)=\left\{\left(x^{2},(0,1, \infty)\right)\right\} .
$$

Wild ramification examples of this sort are closely related to the failure of the classical Riemann-Hurwitz formula in positive characteristic.

Remark 15.5. Note that we can use weighted portraits to easily describe moduli spaces of polynomial maps on $\mathbb{P}^{1}$, since $\mathcal{M}_{d}^{1}[\mathcal{P}]$ parameterizes polynomial maps if and only if $\mathcal{P}=\left(\mathcal{V}^{\circ}, \mathcal{V}, \Phi, \epsilon\right)$ has a vertex $v \in \mathcal{V}^{\circ}$ satisfying $\Phi(v)=v$ and $\epsilon(v)=d$.

Before stating the main result of this section, we need a definition.

Definition 15.6. Let $\mathcal{P}$ be a portrait. We say that a point $(f, \boldsymbol{P}) \in$ $\operatorname{End}_{d}^{N}[\mathcal{P}](\bar{K})$ is a flexible Lattès model for $\mathcal{P}$ if $f \in \operatorname{End}_{d}^{N}(\bar{K})$ is a flexible Lattès map. We refer the reader to [58, Section 6.5] for the definition of flexible Lattès map, but roughly, it means that $f$ comes from a multiplication-by- $m$ map on an elliptic curve. In particular, the locus of flexible Lattès maps in $\mathcal{M}_{d}^{1}$ is empty if $d$ is not a square, and it is a copy of $\mathbb{P}^{1}$ if $d$ is a square. We then define

$$
\operatorname{End}_{d}^{1}[\mathcal{P}]^{\mathrm{nL}}:=\operatorname{End}_{d}^{1}[\mathcal{P}] \backslash(\text { flexible Lattès })
$$

to be the complement of the points corresponding to flexible Lattès models, and similarly for $\mathcal{M}_{d}^{1}[\mathcal{P}]^{\mathrm{nL}}$. 
Theorem 15.7. Let $d \geq 2$, and let $\mathcal{P}=\left(\mathcal{V}^{\circ}, \mathcal{V}, \Phi, \epsilon\right)$ be a portrait such that $\mathcal{M}_{d}^{1}[\mathcal{P}] \neq \emptyset$. Then

$$
\operatorname{dim} \mathcal{M}_{d}^{1}[\mathcal{P}]^{n L}=\operatorname{dim} \mathcal{M}_{d}^{1}-\sum_{v \in \mathcal{V}^{\circ}}(\epsilon(v)-1)+\#\left(\mathcal{V} \backslash \mathcal{V}^{\circ}\right)
$$

Remark 15.8. Since $\mathcal{M}_{d}^{1}[\mathcal{P}]^{\mathrm{nL}}$ and $\mathcal{M}_{d}^{1}$ have dimension 3 less than $\operatorname{End}_{d}^{1}[\mathcal{P}]^{\mathrm{nL}}$ and $\operatorname{End}_{d}^{1}$, respectively, it suffices to prove Theorem 15.7 with $\mathcal{M}$ replaced with End, i.e., to prove that

$$
\operatorname{dim} \operatorname{End}_{d}^{1}[\mathcal{P}]^{\mathrm{nL}}=\operatorname{dim} \operatorname{End}_{d}^{1}-\sum_{v \in \mathcal{V}^{\circ}}(\epsilon(v)-1)+\#\left(\mathcal{V} \backslash \mathcal{V}^{\circ}\right)
$$

Recall that $\operatorname{End}_{d}^{1}[\mathcal{P}]$ is the subvariety of $\operatorname{End}_{d}^{1} \times\left(\mathbb{P}^{1}\right)_{\Delta}^{S}$ determined by the following two conditions 21

$$
\begin{array}{rlrl}
f\left(P_{w}\right) & =P_{\Phi(w)} & & \text { for all } w \in \mathcal{V}^{\circ} . \\
e_{f}\left(P_{w}\right) \geq \epsilon(w) & & \text { for all } w \in \operatorname{Crit}(\mathcal{P}) .
\end{array}
$$

There are $\# \mathcal{V}^{\circ}+\sum_{w \in \mathcal{V}^{\circ}}(\epsilon(w)-1)$ equations given in (68) and (69); since $\operatorname{End}_{d}^{1}[\mathcal{P}]^{\mathrm{nL}}$ is nonempty, this is an upper bound for the codimension of $\operatorname{End}_{d}^{1}[\mathcal{P}]^{\mathrm{nL}}$ in $\operatorname{End}_{d}^{1} \times\left(\mathbb{P}^{1}\right)_{\Delta}^{S}$, hence

$$
\begin{aligned}
& \operatorname{dim} \operatorname{End}_{d}^{1}[\mathcal{P}]^{\mathrm{nL}} \geq \operatorname{dim} \operatorname{End}_{d}^{1}+S-\left(\# \mathcal{V}^{\circ}+\sum_{w \in \mathcal{V}^{\circ}}(\epsilon(w)-1)\right) \\
& =\operatorname{dim} \operatorname{End}_{d}^{1}-\sum_{w \in \mathcal{V}^{\circ}}(\epsilon(w)-1)+\#\left(\mathcal{V} \backslash \mathcal{V}^{\circ}\right) .
\end{aligned}
$$

Thus, in order to complete the proof of Theorem 15.7, it remains to show that

$$
\operatorname{dim} \operatorname{End}_{d}^{1}[\mathcal{P}]^{\mathrm{nL}} \leq \operatorname{dim} \operatorname{End}_{d}^{1}-\sum_{w \in \mathcal{V}^{\circ}}(\epsilon(w)-1)+\#\left(\mathcal{V} \backslash \mathcal{V}^{\circ}\right)
$$

15.1. A Reduction Step. Because our main focus in this section is on critical points and their corresponding critical relations, we specify, for a weighted portrait $\mathcal{P}$, a minimal subportrait of $\mathcal{P}$ that captures the same critical data as $\mathcal{P}$ itself.

\footnotetext{
${ }^{21}$ We remark that if we write $f(x) \in K(x)$ as a rational function, and if we take a point $\alpha \in K$ satisfying $f(\alpha) \neq \infty$, then an inequality $e_{f}(\alpha) \geq \epsilon$ as in (69) simply says that $\left(d^{n} f / d x^{n}\right)(\alpha)=0$ for all $1 \leq n \leq \epsilon-1$.
} 
Definition 15.9. Let $\mathcal{P}=\left(\mathcal{V}, \mathcal{V}^{\circ}, \Phi, \epsilon\right)$ be a portrait. The critically generated subportrait of $\mathcal{P}$ is the subportrait $\mathcal{P}^{\prime}=\left(\mathcal{V}^{\prime}, \mathcal{V}^{\prime \circ}, \Phi^{\prime}, \epsilon^{\prime}\right)$ defined by

$$
\mathcal{V}^{\prime}:=\bigcup_{v \in \operatorname{Crit}(\mathcal{P})} \mathcal{O}_{\Phi}(v), \quad \mathcal{V}^{\prime \circ}:=\mathcal{V}^{\prime} \cap \mathcal{V}^{\circ}, \quad \Phi^{\prime}:=\left.\Phi\right|_{\mathcal{V}^{\prime \circ}}, \quad \epsilon^{\prime}:=\left.\epsilon\right|_{\mathcal{V}^{\prime \circ}}
$$

We say that $\mathcal{P}$ is critically generated $\sqrt{22}$ if $\mathcal{P}^{\prime}=\mathcal{P}$.

We now show that for the purposes of proving Theorem 15.7, it suffices to consider only critically generated portraits.

Lemma 15.10. If Condition (70) holds for all critically generated portraits, then Condition (70) holds for all portraits.

Proof. Let $\mathcal{P}$ be any portrait, and let $\mathcal{P}^{\prime}$ be its critically generated subportrait. By assumption, we have

$$
\operatorname{dim} \operatorname{End}_{d}^{1}\left[\mathcal{P}^{\prime}\right]^{\mathrm{nL}} \leq \operatorname{dim} \operatorname{End}_{d}^{1}-\sum_{v \in \mathcal{V}^{\prime \circ}}\left(\epsilon^{\prime}(v)-1\right)+\#\left(\mathcal{V}^{\prime} \backslash \mathcal{V}^{\prime \circ}\right)
$$

Consider the natural projection map (Proposition 7.8) restricted to the non-Lattès locus,

$$
\pi_{\mathcal{P}, \mathcal{P}^{\prime}}: \operatorname{End}_{d}^{N}[\mathcal{P}]^{\mathrm{nL}} \longrightarrow \operatorname{End}_{d}^{N}\left[\mathcal{P}^{\prime}\right]^{\mathrm{nL}}
$$

As in the proof of Theorem 14.8, the dimension of a nonempty fiber of $\pi_{\mathcal{P}, \mathcal{P}^{\prime}}$ is equal to $\#\left(\mathcal{V} \backslash \mathcal{V}^{\circ}\right)-\#\left(\mathcal{V}^{\prime} \backslash \mathcal{V}^{\prime \circ}\right)$. Thus, noting that

$$
\sum_{v \in \mathcal{V}^{\prime \circ}}\left(\epsilon^{\prime}(v)-1\right)=\sum_{v \in \mathcal{V}^{\circ}}(\epsilon(v)-1)
$$

by construction of $\mathcal{P}^{\prime}$, we have

$$
\begin{aligned}
\operatorname{dim} \operatorname{End}_{d}^{1}[\mathcal{P}]^{\mathrm{nL}} & =\operatorname{dim} \operatorname{Image}\left(\pi_{\mathcal{P}, \mathcal{P}^{\prime}}\right)+\operatorname{dim} \operatorname{Fiber}\left(\pi_{\mathcal{P}, \mathcal{P}^{\prime}}\right) \\
& \leq \operatorname{dim} \operatorname{End}_{d}^{1}\left[\mathcal{P}^{\prime}\right]^{\mathrm{nL}}+\left(\#\left(\mathcal{V} \backslash \mathcal{V}^{\circ}\right)-\#\left(\mathcal{V}^{\prime} \backslash \mathcal{V}^{\circ}\right)\right) \\
& \leq \operatorname{dim} \operatorname{End}_{d}^{1}-\sum_{v \in \mathcal{V}^{\circ}}(\epsilon(v)-1)+\#\left(\mathcal{V} \backslash \mathcal{V}^{\circ}\right)
\end{aligned}
$$

so (170) holds for $\mathcal{P}$.

Remark 15.11. In the proof of Lemma 15.10, we did not assume that $\mathcal{P}$ has a vertex with weight greater than 1 . If $\mathcal{P}$ is unweighted, i.e., if $\epsilon(v)=1$ for all $v \in \mathcal{V}^{\circ}$, then the critically generated subportrait $\mathcal{P}^{\prime}$ is the empty portrait, and in which case we have $\operatorname{End}_{d}^{1}\left[\mathcal{P}^{\prime}\right]=\operatorname{End}_{d}^{1}[\emptyset]=$ $\operatorname{End}_{d}^{1}$. The argument is no different in this situation.

${ }^{22}$ If $\sum_{v \in \mathcal{V}^{\circ}}(\epsilon(v)-1)=2 d-2$, then $\mathcal{P}$ being critically generated is equivalent to $\mathcal{P}$ being a complete critical portrait, using the terminology of Section 12 


\subsection{Critical Relations Realized by Critically Generated Por-} traits. For the remainder of this section, we assume that $\mathcal{P}$ is a critically generated portrait.

In order to prove Theorem 15.7, we relabel vertices of $\mathcal{P}$ using pairs of positive integers as follows: First, we decompose $\mathcal{V}$ as a disjoint union

$$
\mathcal{V}=\mathcal{C}_{1} \cup \cdots \cup \mathcal{C}_{r}
$$

consisting of the vertices in the distinct connected components of the graph attached to $\mathcal{P}$. Note that every component contains at least one critical point by our assumption that $\mathcal{P}$ is critically generated.

For each $1 \leq k \leq r$, we label the elements of $\mathcal{C}_{k}$ as

$$
\mathcal{C}_{k}=\left\{(1, k), \ldots,\left(s_{k}, k\right)\right\},
$$

and we choose the labeling so that there is a $t_{k} \leq s_{k}$ so that

$$
(i, k) \in \mathcal{C}_{k} \text { is a critical point } \Longleftrightarrow 1 \leq i \leq t_{k} .
$$

In other words, we have

$$
\begin{aligned}
\mathcal{V} & =\left\{(i, k): 1 \leq k \leq r, 1 \leq i \leq s_{k}\right\} \text { and } \\
\operatorname{Crit}(\mathcal{P}) & =\left\{(i, k): 1 \leq k \leq r, 1 \leq i \leq t_{k}\right\} .
\end{aligned}
$$

Let

$$
S:=\sum_{k=1}^{r} s_{k} \quad \text { and } \quad T:=\sum_{k=1}^{r} t_{k}
$$

be, respectively, the total number of vertices in $\mathcal{V}$ and the total number of critical points for $\mathcal{V}$. Finally, we let

$$
\zeta(\mathcal{P}):=\#\left(\mathcal{V} \backslash \mathcal{V}^{\circ}\right)
$$

be the number of components of $\mathcal{P}$ that do not contain cycle.

Definition 15.12. A critical relation realized by $\mathcal{P}$ is an identity of the form

$$
\Phi^{m}(i, k)=\Phi^{n}(j, \ell)
$$

with $(i, k),(j, \ell) \in \operatorname{Crit}(\mathcal{P})$. We also encode such a relation as a tuple

$$
((i, k),(j, \ell) ; m, n) \text {. }
$$

The relation is defined to be trivial if $(i, k)=(j, \ell)$ and $m=n$, and nontrivial otherwise.

Note that for any critical relation (72), we must have $k=\ell$, since otherwise the vertices $(i, k)$ and $(j, \ell)$ would be in different components.

We seek to construct a minimal set of critical relations realized by $\mathcal{P}$ that completely determines all relations realized by $\mathcal{P}$. First, we make 
more explicit what we mean for a set of critical relations to determine another relation. The following definition is motivated by [37, $\S 3.1$ ].

Definition 15.13. Let $\mathcal{S}$ be a collection of critical relations realized by $\mathcal{P}$. We let $\sim_{\mathcal{S}}$ denote the smallest equivalence relation $\sim$ on the set

$$
\left\{((i, k), m) \mid 1 \leq k \leq r, 1 \leq i \leq t_{k}, m \in \mathbb{Z}_{\geq 0}\right\}
$$

with the property that

$$
\begin{aligned}
((i, k), m) & \sim((j, \ell), n) \\
& \Longrightarrow((i, k), m+c) \sim((j, \ell), n+c) \text { for all } c \in \mathbb{Z}_{\geq 0} .
\end{aligned}
$$

We refer to the condition (73) by saying that $\sim_{\mathcal{S}}$ is closed under iteration. We say that a relation $((i, k),(j, \ell) ; m, n)$ as in (72) is determined by $\mathcal{S}$ if $((i, k), m) \sim_{\mathcal{S}}((j, \ell), n)$.

We now define a particular set of critical relations for $\mathcal{P}$, which we will then show determines all critical relations realized by $\mathcal{P}$.

Definition 15.14. We construct a set $\mathcal{S}_{\mathcal{P}}$ using the following algorithm:

(A) Initialize $\mathcal{S}_{\mathcal{P}}:=\emptyset, k:=1$, and $i:=1$.

(B) If $i=1$ and the component $\mathcal{C}_{k}$ has no cycle, skip to Step $(\mathrm{G})$.

(C) Let $m_{i, k}$ be the minimal $m \geq 0$ such that $\Phi^{m}(i, k)=\Phi^{n}(j, k)$ for some $1 \leq j \leq i$ and $n \geq 0$, with $n<m$ if $i=j$. (Step (B) ensures that there is such an $m$.)

(D) Let $j_{i, k}$ be the minimal $1 \leq j \leq i$ such that $\Phi^{m_{i, k}}(i, k)=\Phi^{n}(j, k)$ for some $n \geq 0$.

(E) Let $n_{i, k}$ be the minimal $n \geq 0$ such that $\Phi^{m_{i, k}}(i, k)=\Phi^{n}\left(j_{i, k}, k\right)$.

(F) Adjoin the tuple $\left((i, k),\left(j_{i, k}, k\right) ; m_{i, k}, n_{i, k}\right)$ to the set $\mathcal{S}_{\mathcal{P}}$.

(G) Increment $i$. If $i \leq t_{k}$, go to Step (C).

(H) Increment $k$. If $k \leq r$, go to Step (B).

We recall the quantity $\zeta(\mathcal{P})$ defined by (71), and we note that the set $\mathcal{S}_{\mathcal{P}}$ contains precisely $T-\zeta(\mathcal{P})$ tuples, since $\operatorname{Step}(\mathrm{B})$ eliminates $\zeta(\mathcal{P})$ of the critical points. We now show that $\mathcal{S}_{\mathcal{P}}$ determines all critical relations for $\mathcal{P}$.

Proposition 15.15. Let $\mathcal{S}_{\mathcal{P}}$ be as in Definition 15.14. Then every critical relation $\Phi^{m}(i, k)=\Phi^{n}(j, \ell)$ realized by $\mathcal{P}$ is determined by $\mathcal{S}_{\mathcal{P}}$.

Proof. We drop the subscript and write $\sim$ for the equivalence relation $\sim_{\mathcal{S}_{\mathcal{P}}}$. Since $\sim$ is reflexive, any trivial relation is immediately determined by $\mathcal{S}_{\mathcal{P}}$. Henceforth, we consider only nontrivial critical relations. 
As noted previously, for any critical relation $\Phi^{m}(i, k)=\Phi^{n}(j, \ell)$ we must have $k=\ell$. Now, fix $k \in\{1, \ldots, r\}$, and assume that $i \geq j$. We proceed by induction on $i$.

First, we take $i=1$, hence also $j=1$. There is a nontrivial critical relation of the form $\Phi^{m}(1, k)=\Phi^{n}(1, k)$ if and only if the component $\mathcal{C}_{k}$ contains a cycle. We assume that $m \geq n$. In this case, if we let $(M, N)$ denote the preperiodic type of $(1, k)$, then we must have $n \geq M$ and $m=n+c N$ for some $c \geq 1$. (If $c=0$, then the relation is trivial.) Therefore, it suffices to show that $((1, k), n) \sim((1, k), n+c N)$ for all integers $n \geq M$ and $c \geq 1$.

Using the notation from Definition 15.14 we have

$$
j_{1, k}=1, \quad m_{1, k}=M+N, \quad n_{1, k}=M .
$$

Thus $((1, k), M) \sim((1, k), M+N)$. Since $n \geq M$, and since $\sim$ is closed under iteration, it follows also that $((1, k), n) \sim((1, k), n+N)$. In fact, we have $((1, k), n+(c-1) N) \sim((1, k), n+c N)$ for all $c \geq 1$, hence by transitivity we have $((1, k), n) \sim((1, k), n+c N)$ for all $c \geq 1$ as claimed.

Now take $2 \leq i \leq t_{k}$, and assume that for all $1 \leq j^{\prime} \leq i^{\prime}<$ $i$, if $\Phi^{m^{\prime}}\left(i^{\prime}, k\right)=\Phi^{n^{\prime}}\left(j^{\prime}, k\right)$, then that relation is determined by $\mathcal{S}_{\mathcal{P}}$. Since $i \geq 2$, we must have $j_{i, k}<i$. We consider two cases.

Case 1. Assume $i=j$, in which case $\mathcal{C}_{k}$ contains a cycle. Let $(M, N)$ be the preperiodic type of $(i, k)$. By following the same argument as in the $i=1$ case, it will suffice to show that $((i, k), M) \sim((i, k), M+N)$.

The vertex $\Phi^{M}(i, k)$ lies in the $N$-cycle in $\mathcal{C}_{k}$, which is contained in the orbit of $(1, k)$; thus $m_{i, k} \leq M$. By definition of $\mathcal{S}_{\mathcal{P}}$, we have

$$
\left((i, k), m_{i, k}\right) \sim\left(\left(j_{i, k}, k\right), n_{i, k}\right),
$$

and by closure under iteration we have

$$
((i, k), M) \sim\left(\left(j_{i, k}, k\right), n_{i, k}+M-m_{i, k}\right)
$$

and

$$
((i, k), M+N) \sim\left(\left(j_{i, k}, k\right), n_{i, k}+M-m_{i, k}+N\right) .
$$

It therefore suffices to show that

$$
\left(\left(j_{i, k}, k\right), n_{i, k}+M-m_{i, k}\right) \sim\left(\left(j_{i, k}, k\right), n_{i, k}+M-m_{i, k}+N\right) .
$$

Now, by construction we have that $\Phi^{n_{i, k}}\left(j_{i, k}, k\right)=\Phi^{m_{i, k}}(i, k)$; thus, since $M \geq m_{i, k}$, we have

$$
\Phi^{M-m_{i, k}+n_{i, k}}\left(j_{i, k}, k\right)=\Phi^{M}(i, k),
$$

which is periodic of period $N$. It follows that

$$
\Phi^{M-m_{i, k}+n_{i, k}+N}\left(j_{i, k}, k\right)=\Phi^{M-m_{i, k}+n_{i, k}}\left(j_{i, k}, k\right) .
$$


Since $j_{i, k}<i$, our induction assumption guarantees that (74) holds.

Case 2. Assume $i>j$. Since $\Phi^{m}(i, k)=\Phi^{n}(j, k)$, we have $m_{i, k} \leq m$ by definition, and furthermore

$$
\left((i, k), m_{i, k}\right) \sim\left(\left(j_{i, k}, k\right), n_{i, k}\right) .
$$

By closure under iteration, since $m \geq m_{i, k}$ we get

$$
((i, k), m) \sim\left(\left(j_{i, k}, k\right), n_{i, k}+m-m_{i, k}\right) .
$$

On the other hand, we have

$$
\Phi^{n_{i, k}+m-m_{i, k}}\left(j_{i, k}, k\right)=\Phi^{m}(i, k)=\Phi^{n}(j, k),
$$

and since $j_{i, k}, j<i$, our induction hypothesis implies that

$$
\left(\left(j_{i, k}, k\right), n_{i, k}+m-m_{i, k}\right) \sim((j, k), n) .
$$

By transitivity, we get $((i, k), m) \sim((j, k), n)$, as claimed.

15.3. Proof of Theorem [15.7. The key to proving inequality (70), and therefore Theorem [15.7, is to note that the relations coming from (68) and (69) intersect transversally at points in $\operatorname{End}_{d}^{1}[\mathcal{P}]$. This, in turn, relies on Thurston rigidity; see [15] and [37].

Proof of Theorem 15.7. Lemma 15.10 says that it suffices to assume that $\mathcal{P}$ is critically generated, and thus we need to show that (70) is satisfied by such $\mathcal{P}$.

Let

$$
\left(f,\left(P_{i, k}\right)_{\substack{1 \leq k \leq r \\ 1 \leq i \leq s_{k}}}\right) \in \operatorname{End}_{d}^{1}[\mathcal{P}]^{\mathrm{nL}}
$$

The portrait $\mathcal{P}$ determines $T$ distinct critical points for $f$, accounting for at least

$$
\sum_{k=1}^{r} \sum_{i=1}^{t_{k}}(\epsilon(i, k)-1)
$$

critical points when counted with multiplicity. This means that the map $f$ has at most

$$
T^{\prime}:=T+2 d-2-\sum_{k=1}^{r} \sum_{i=1}^{t_{k}}(\epsilon(i, k)-1)
$$

distinct critical points. By [37, Theorem 2.1], the (generally reducible) subvariety $\operatorname{End}_{d, T^{\prime}}^{1} \subset \operatorname{End}_{d}^{1}$ of maps with at most $T^{\prime}$ distinct critical points has dimension at most $T^{\prime}+3$. It follows that the subvariety $\mathcal{X} \subset \operatorname{End}_{d}^{1} \times\left(\mathbb{P}^{1}\right)_{\Delta}^{T}$ defined by

$$
e_{f}\left(P_{i, k}\right) \geq \epsilon(i, k) \quad \text { for all } 1 \leq k \leq r, 1 \leq i \leq t_{k},
$$


has dimension at most $T^{\prime}+3$, since the projection map $\mathcal{X} \rightarrow \operatorname{End}_{d}^{1}$ has finite fibers, and its image lies in $\operatorname{End}_{d, T^{\prime}}^{1}$.

Let $\mathcal{Z}$ be the subvariety of $\mathcal{X}$ cut out by

$$
f^{m}\left(P_{i, k}\right)=f^{n}\left(P_{j, \ell}\right) \quad \text { for all }((i, k),(j, \ell) ; m, n) \in \mathcal{S}_{\mathcal{P}} .
$$

Then the image of the map $\pi_{\mathcal{X}}: \operatorname{End}_{d}^{1}[\mathcal{P}] \rightarrow \mathcal{X}$ obtained by projecting onto the appropriate coordinates is contained in $\mathcal{Z}$. Furthermore, by construction of $\mathcal{S}_{\mathcal{P}}$, there can be no redundancy among the relations; that is, there is no subset of distinct elements of $\mathcal{S}_{\mathcal{P}}$ of the form

$$
\begin{aligned}
& \left(\left(i_{1}, k\right),\left(i_{2}, k\right) ; m_{1}, n_{1}\right),\left(\left(i_{2}, k\right),\left(i_{3}, k\right) ; m_{2}, n_{2}\right), \\
& \ldots,\left(\left(i_{\ell-1}, k\right),\left(i_{\ell}, k\right) ; m_{\ell-1}, n_{\ell-1}\right),\left(\left(i_{\ell}, k\right),\left(i_{1}, k\right) ; m_{\ell}, n_{\ell}\right) .
\end{aligned}
$$

Thus, Thurston rigidity implies 23 that the relations defining $\mathcal{Z}$ intersect transversally in $\mathcal{X}$ at each non-Lattés point in Image $\left(\pi_{\mathcal{X}}\right)$. Therefore, we have

$$
\begin{aligned}
\operatorname{dim} \operatorname{Image}\left(\left.\pi_{\mathcal{X}}\right|_{\left.\operatorname{End}_{d}^{1}[\mathcal{P}]^{\mathrm{nL}}\right)}\right) & \operatorname{dim} \mathcal{X}-\# \mathcal{S}_{\mathcal{P}} \\
& \leq\left(T^{\prime}+3\right)-(T-\zeta(\mathcal{P})) \\
& =\operatorname{dim} \operatorname{End}_{d}^{1}-\sum_{k=1}^{r} \sum_{i=1}^{t_{k}}(\epsilon(i, k)-1)+\zeta(\mathcal{P}) \\
& =\operatorname{dim} \operatorname{End}_{d}^{1}-\sum_{v \in \mathcal{V}^{\circ}}(\epsilon(v)-1)+\#\left(\mathcal{V} \backslash \mathcal{V}^{\circ}\right),
\end{aligned}
$$

where the last equality comes from the fact that we defined $\zeta(\mathcal{P})$ to be equal to $\#\left(\mathcal{V} \backslash \mathcal{V}^{\circ}\right)$. The fact that $\mathcal{P}$ is critically generated implies that for every vertex $(j, \ell) \in \mathcal{V}$, there exist $(i, k) \in \operatorname{Crit}(\mathcal{P})$ and $m \geq 0$ such that $\Phi^{m}(i, k)=(j, \ell)$. This implies that $P_{j, \ell}=f^{m}\left(P_{i, k}\right)$, and thus every coordinate $P_{j, \ell}$ is determined by just the critical point coordinates. It follows that $\operatorname{End}_{d}^{1}[\mathcal{P}]^{\mathrm{nL}}$ is isomorphic to its image under $\pi_{\mathcal{X}}$, and therefore the inequality (76) implies (70).

\section{An FOD/FOM Degree Bound for Dynamical Systems With PORTRAits}

In this section, we turn to a discussion of the field-of-moduli versus field-of-definition problem associated to the space $\mathcal{M}_{d}^{N}[\mathcal{P}]$ with an unweighted portrait $\mathcal{P}$. In the next section we apply this material to

\footnotetext{
${ }^{23}$ More explicitly, one can extend $\mathcal{S}_{\mathcal{P}}$ to a larger set of relations $\mathcal{S}_{f} \supseteq \mathcal{S}_{\mathcal{P}}$ which is minimally full in the sense of [37, Definition 3.6], and then transversality follows from [37, Lemma 4.1] and the proof of [37, Theorem 3.2].
} 
explain how the uniform boundedness conjecture is related to rational points on $\mathcal{M}_{d}^{N}[\mathcal{P}]$.

The main theorem of 19 says that if $K$ is a number field and $\xi \in$ $\mathcal{M}_{d}^{N}(K)$, then there is a field extension $L / K$ whose degree is bounded in terms of $N$ and $d$ so that $\xi$ comes from a point in $\operatorname{End}_{d}^{N}(L)$. We generalize to portrait moduli spaces $\mathcal{M}_{d}^{N}[\mathcal{P}]$, obtaining a result that will be needed in the next section for comparing two uniform boundedness conjectures.

Theorem 16.1. Fix integers $N \geq 1$ and $d \geq 2$. There is a constant $C_{2}(N, d)$ such that for every unweighted portrait $\mathcal{P}$ and every number field $K$ we have

$$
\mathcal{M}_{d}^{N}[\mathcal{P}](K) \subset \operatorname{Image}\left(\bigcup_{[L: K] \leq C_{2}(N, d)} \operatorname{End}_{d}^{N}[\mathcal{P}](L) \longrightarrow \mathcal{M}_{d}^{N}[\mathcal{P}](\bar{K})\right) .
$$

In other words, every $\xi \in \mathcal{M}_{d}^{N}[\mathcal{P}](\bar{K})$ whose field of moduli is contained in $K$ has a field of definition whose degree over $K$ is bounded in terms of $N$ and $d$.

Proof. Recall that we use angle brackets to denote the maps

$$
\langle\cdot\rangle: \operatorname{End}_{d}^{N}[\mathcal{P}] \longrightarrow \mathcal{M}_{d}^{N}[\mathcal{P}] \text { and }\langle\cdot\rangle: \operatorname{End}_{d}^{N} \longrightarrow \mathcal{M}_{d}^{N} .
$$

We start with an element $\langle f, \boldsymbol{P}\rangle \in \mathcal{M}_{d}^{N}[\mathcal{P}](K)$, i.e., the field of moduli of $(f, \boldsymbol{P})$ is contained in $K$. Then $\langle f\rangle \in \mathcal{M}_{d}^{N}(K)$, so [19, Theorem 1] says that there is a $\psi \in \mathrm{PGL}_{N+1}(\bar{K})$ so that $f^{\psi} \in \operatorname{End}_{d}^{N}(L)$ for a field $L$ whose degree over $K$ is bounded in terms of $N$ and $d$. Replacing $K$ with $L$ and $(f, \boldsymbol{P})$ with $\left(f^{\psi}, \psi^{-1}(\boldsymbol{P})\right)$, we may assume without loss of generality that $\langle f, \boldsymbol{P}\rangle \in \mathcal{M}_{d}^{N}[\mathcal{P}](K)$ and $f \in \operatorname{End}_{d}^{N}(K)$.

It follows that for all $\sigma \in \operatorname{Gal}(\bar{K} / K)$, we have

$$
\left\langle f, \boldsymbol{P}^{\sigma}\right\rangle=\left\langle f^{\sigma}, \boldsymbol{P}^{\sigma}\right\rangle=\langle f, \boldsymbol{P}\rangle^{\sigma}=\langle f, \boldsymbol{P}\rangle .
$$

Hence there is a exists $\varphi_{\sigma} \in \operatorname{PGL}_{N+1}(\bar{K})$ such that

$$
\left(f, \boldsymbol{P}^{\sigma}\right)=\varphi_{\sigma} \star(f, \boldsymbol{P})=\left(f^{\varphi_{\sigma}}, \varphi_{\sigma}^{-1}(\boldsymbol{P})\right) .
$$

In particular, we see that $\varphi_{\sigma} \in \operatorname{Aut}(f)$, and hence

$$
\#\left\{\boldsymbol{P}^{\sigma}: \sigma \in \operatorname{Gal}(\bar{K} / K)\right\} \leq \#\left\{\varphi^{-1} \boldsymbol{P}: \varphi \in \operatorname{Aut}(f)\right\} \leq \# \operatorname{Aut}(f) .
$$

It follows that

$$
[K(\boldsymbol{P}): K] \leq \# \operatorname{Aut}(f),
$$

and now we need merely invoke Levy's theorem [38, mentioned already in the proof of Theorem5.1(a), that \# Aut $(f)$ is bounded by a constant that depends on only $N$ and $d$. 


\section{Uniform Boundedness of Preperiodic Points and Its Relation to Algebraic Points on $\mathcal{M}_{d}^{N}[\mathcal{P}]$}

The strong uniform boundedness conjecture gives a strong uniform bound for the number of preperiodic points of morphisms of $\mathbb{P}^{N}$ over number fields of bounded degree. In this section we relate this conjecture to the existence of algebraic points on moduli spaces $\mathcal{M}_{d}^{N}[\mathcal{P}]$.

Conjecture 17.1 (Strong Uniform Boundedness Conjecture; Silverman-Morton [49]). For every $D \geq 1, N \geq 1$, and $d \geq 2$ there is a constant $C_{3}(D, N, d)$ such that for all number fields $K / \mathbb{Q}$ satisfying $[K: \mathbb{Q}] \leq D$ and all endomorphisms $f \in \operatorname{End}_{d}^{N}(K)$, we have

$$
\#\left(\operatorname{PrePer}(f) \cap \mathbb{P}^{N}(K)\right) \leq C_{3}(D, N, d) .
$$

Remark 17.2. Fakhruddin [21] has shown that Conjecture 17.1 implies an analogous uniform boundedness theorem for torsion points on abelian varieties. He has also shown that if Conjecture 17.1 is true for $D=1$, i.e., for $K=\mathbb{Q}$, then it is true for all number fields. Levy, Manes, and Thompson [39, Theorem 2.9] have shown that Conjecture 17.1 is true if we fix a map $f$ and consider only the family of $\bar{K} / K$-twists of $f$, i.e., the set of maps $f^{\varphi}$ such that $\varphi \in \operatorname{PGL}_{N+1}(\bar{K})$ and $f^{\varphi} \in \operatorname{End}_{d}^{N}(K)$.

We next consider a uniformity conjecture for points on $\mathcal{M}_{d}^{N}[\mathcal{P}]$ over fields of bounded degree.

Conjecture 17.3 (Strong Moduli Boundedness Conjecture). For every $D \geq 1, N \geq 1$, and $d \geq 2$ there is a constant $C_{4}(D, N, d)$ such that for all number fields $K / \mathbb{Q}$ satisfying $[K: \mathbb{Q}] \leq D$ and all unweighted preperiodic portraits $\mathcal{P}=(\mathcal{V}, \Phi)$ satisfying $\# \mathcal{V} \geq C_{4}(D, N, d)$, we have

$$
\mathcal{M}_{d}^{N}[\mathcal{P}](K)=\emptyset
$$

Example 17.4. Let $\mathcal{C}_{n}$ denote an unweighted portrait containing a single $n$-cycle, and let $\mathcal{P}_{1}$ and $\mathcal{P}_{2}$ be the following portraits:

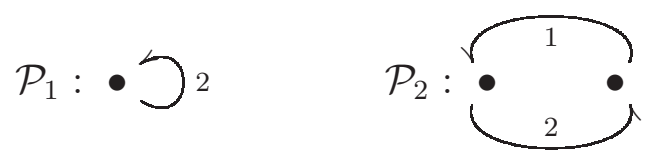

Flynn, Poonen, and Schaefer [23] conjecture that $\mathcal{M}_{2}^{1}\left[\mathcal{P}_{1} \sqcup \mathcal{C}_{n}\right](\mathbb{Q})=\emptyset$ for all $n \geq 4$, or equivalently, that a polynomial of the form $x^{2}+c$ with $c \in \mathbb{Q}$ has no $\mathbb{Q}$-rational periodic points of period 4 or greater. Assuming this conjecture, Poonen [53] proves that there are exactly 12 preperiodic portraits $\mathcal{P}_{1}^{\prime} \supseteq \mathcal{P}_{1}$ (up to isomorphism) with $\mathcal{M}_{2}^{1}\left[\mathcal{P}_{1}^{\prime}\right](\mathbb{Q}) \neq$ 
$\emptyset$, and that among these portraits, the maximum number of vertices is 9 .

Similarly, Canci and Vishkautsan [8] conjecture that for all $n \geq 2$ we have $\mathcal{M}_{2}^{1}\left[\mathcal{P}_{2} \sqcup \mathcal{C}_{n}\right](\mathbb{Q})=\emptyset$, i.e., if $f \in \mathcal{M}_{2}^{1}(\mathbb{Q})$ has a critical 2cycle, then $f$ contains no further $\mathbb{Q}$-rational non-fixed periodic points. Assuming this conjecture, they prove that there are exactly 13 possible preperiodic portraits $\mathcal{P}_{2}^{\prime} \supseteq \mathcal{P}_{2}$ (up to isomorphism) with $\mathcal{M}_{2}^{1}\left[\mathcal{P}_{2}^{\prime}\right](\mathbb{Q}) \neq$ $\emptyset$, and that among these portraits, the maximum number of vertices is 9 .

As one might expect, Conjectures 17.1 and 17.3 are equivalent, but the proof involves a subtlety due to field of moduli versus field of definition issues.

Theorem 17.5. Conjecture 17.1 is true if and only if Conjecture 17.3 is true.

Proof. The logic of the proof of Theorem 17.5 is as follows:

(I): (Conjecture 17.1) AND NOT(Conjecture 17.3) $\Longrightarrow \rightarrow \leftarrow$.

(II): (Conjecture 17.3) AND NOT(Conjecture 17.1) $\Longrightarrow \rightarrow \leftarrow$.

Since the statements of the two conjectures involve a lot of quantifiers, we take a moment to succinctly state them and their converses, where to ease notation we assume that all portraits are preperiodic and unweighted; see Figure 2 .

\begin{tabular}{|c|}
\hline Conjecture 17.1 (Strong Uniform Boundedness Conjecture) \\
$\forall D \geq 1 \forall N \geq 1 \forall d \geq 2 \exists C_{3} \forall K / \mathbb{Q},[K: \mathbb{Q}] \leq D$ \\
$\forall f \in \operatorname{End}_{d}^{N}(K): \#\left(\operatorname{PrePer}(f) \cap \mathbb{P}^{N}(K)\right) \leq C_{3}$. \\
\hline Converse of Conjecture $\mathbf{1 7 . 1}$ \\
$\exists D \geq 1 \exists N \geq 1 \exists d \geq 2 \forall B \exists K / \mathbb{Q},[K: \mathbb{Q}] \leq D$ \\
$\exists f \in \operatorname{End}_{d}^{N}(K): \#\left(\operatorname{PrePer}(f) \cap \mathbb{P}^{N}(K)\right) \geq B$. \\
\hline Conjecture $\mathbf{1 7 . 3}($ Strong Moduli Boundedness Conjecture) \\
$\forall D \geq 1 \forall N \geq 1 \forall d \geq 2 \exists C_{4} \forall K / \mathbb{Q},[K: \mathbb{Q}] \leq D$ \\
$\forall \mathcal{P}=(\mathcal{V}, \Phi), \# \mathcal{V} \geq C_{4}: \mathcal{M}_{d}^{N}[\mathcal{P}](K)=\emptyset$. \\
Converse of Conjecture $[\mathbf{1 7 . 3}$ \\
$\exists D \geq 1 \exists N \geq 1 \exists d \geq 2 \forall B \exists K / \mathbb{Q},[K: \mathbb{Q}] \leq D$ \\
$\exists \mathcal{P}=(\mathcal{V}, \Phi), \# \mathcal{V} \geq B: \mathcal{M}_{d}^{N}[\mathcal{P}](K) \neq \emptyset$. \\
\hline
\end{tabular}

Figure 2. Conjectures 17.1 and 17.3 and their converses 
Part I: In the converse to Conjecture 17.3, we fix the $(D, N, d)$ whose existence is asserted. Then for each $B$, the field $K$ and portrait $\mathcal{P}$ depend on the choice of $B$, so we label them $K_{B}$ and $\mathcal{P}_{B}=\left(\mathcal{V}_{B}, \Phi_{B}\right)$ to indicate this dependence. The converse of Conjecture 17.3 tells us that

$$
\left[K_{B}: \mathbb{Q}\right] \leq D, \quad \# \mathcal{V}_{B} \geq B, \quad \text { and } \quad \mathcal{M}_{d}^{N}\left[\mathcal{P}_{B}\right]\left(K_{B}\right) \neq \emptyset .
$$

Let $\xi_{B} \in \mathcal{M}_{d}^{N}\left[\mathcal{P}_{B}\right]\left(K_{B}\right)$. Theorem 16.1 says that there is a field of definition $L_{\xi_{B}} / K$ for $\xi_{B}$ whose degree satisfies

$$
\left[L_{\xi_{B}}: K\right] \leq C_{2}(N, d) \text {. }
$$

We fix a representative $\bar{\xi}_{B}$ for $\xi_{B}$ that is defined over $L_{\xi_{B}}$, say

$$
\bar{\xi}_{B}=\left(f, P_{1}, \ldots, P_{n}\right) \in \operatorname{End}_{d}^{N}\left[\mathcal{P}_{B}\right]\left(L_{\xi_{B}}\right),
$$

where $n=\# \mathcal{V}_{B}$. The fact that $\bar{\xi}_{B}$ is defined over $L_{\xi_{B}}$ means that $f \in$ $\operatorname{End}_{d}^{N}\left(L_{\xi_{B}}\right)$ and $P_{1}, \ldots, P_{n} \in \mathbb{P}^{N}\left(L_{\xi_{B}}\right)$, and our assumption that $\mathcal{P}_{B}$ is a preperiodic portrait means that $P_{1}, \ldots, P_{n}$ are in $\operatorname{PrePer}(f)$. Hence

$$
\#\left(\operatorname{PrePer}(f) \cap \mathbb{P}^{N}\left(L_{\xi_{B}}\right)\right) \geq \# \mathcal{V}_{B} \geq B \text {. }
$$

On the other hand, we have

$$
\left[L_{\xi_{B}}: \mathbb{Q}\right]=\left[L_{\xi_{B}}: K\right] \cdot[K: \mathbb{Q}] \leq C_{2}(N, d) \cdot D .
$$

We now assume that Conjecture 17.1 is true and derive a contradiction. Applying Conjecture 17.1 with the field $L_{\xi_{B}}$ in place of $K$ and the degree constant $C_{2}(N, d) \cdot D$ in place of $D$, we find that

$$
\#\left(\operatorname{PrePer}(f) \cap \mathbb{P}^{N}\left(L_{\xi_{B}}\right)\right) \leq C_{3}\left(C_{2}(N, d) \cdot D, N, d\right) .
$$

Combining (77) and (78) yields

$$
C_{3}\left(C_{2}(N, d) \cdot D, N, d\right) \geq B
$$

We have proven that this inequality is true for some $(D, N, d)$ and all $B$, which is impossible. This completes the proof that Conjecture 17.1 and the converse of Conjecture 17.3 give a contradiction.

Part II: We assume that Conjecture 17.1 is false, and we fix $(D, N, d)$ whose existence is asserted in the converse to Conjecture 17.1. Then for every choice of $B$, the field $K$ and endomorphism $f$ in the converse to Conjecture 17.1 depend on the choice of $B$, so we label them $K_{B}$ and $f_{B}$ to indicate this dependence. They satisfy

$$
\left[K_{B}: \mathbb{Q}\right] \leq D, \quad f_{B} \in \operatorname{End}_{d}^{N}\left(K_{B}\right), \quad \#\left(\operatorname{PrePer}\left(f_{B}\right) \cap \mathbb{P}^{N}\left(K_{B}\right)\right) \geq B .
$$


We label the $K_{B}$-rational preperiodic points of $f_{B}$ as

$$
\operatorname{PrePer}\left(f_{B}\right) \cap \mathbb{P}^{N}\left(K_{B}\right)=\left\{P_{1}, \ldots, P_{n}\right\},
$$

and we let $\mathcal{P}_{B}=\left(\mathcal{V}_{B}, \Phi_{B}\right)$ be the abstract portrait determined by the action of $f_{B}$ on $\left(P_{1}, \ldots, P_{n}\right)$. Then

$$
\bar{\xi}_{B}:=\left(f, P_{1}, \ldots, P_{n}\right) \in \operatorname{End}_{d}^{N}\left[\mathcal{P}_{B}\right]\left(K_{B}\right),
$$

and in particular $\mathcal{M}_{d}^{N}\left[\mathcal{P}_{B}\right]\left(K_{B}\right) \neq \emptyset$.

We are also assuming that Conjecture 17.3 is true, and Conjecture 17.3 tells us that if $\# \mathcal{V} \geq C_{4}(D, N, d)$, then $\mathcal{M}_{d}^{N}\left[\mathcal{P}_{B}\right]\left(K_{B}\right)$ would be empty. We conclude that $\# \mathcal{V}<C_{4}(D, N, d)$. To recapitulate, we have proven that there exists a triple $(D, N, d)$ such that for all $B$,

$$
C_{4}(D, N, d) \geq \# \mathcal{V}=n=\#\left(\operatorname{PrePer}\left(f_{B}\right) \cap \mathbb{P}^{N}\left(K_{B}\right)\right) \geq B .
$$

This is clearly false, which completes the proof that Conjecture 17.3 and the converse of Conjecture 17.1 lead to a contradiction.

\section{Good Reduction of Dynamical Systems}

Good reduction for dynamical systems with portraits, which is the topic of the paper 56, is closely related to the existence of integral points on the moduli spaces constructed in the present paper. We discuss this connection, and refer the reader to [56] for further details, especially for the dimension 1 case.

Definition 18.1. Let $K$ be a number field with ring of integers $R$, let $S$ be a finite set of places of $K$, including all archimedean places, and let $R_{S}$ be the ring of $S$-integers of $K$.

A map $f \in \operatorname{End}_{d}^{N}(K)$ has good reduction outside of $S$ if $\langle f\rangle$ is in the image of the map

$$
\operatorname{End}_{d}^{N}\left(R_{S}\right) \longrightarrow \mathcal{M}_{d}^{N}(K) .
$$

This is equivalent to there being a change of variables so that the new $f$ induces a morphism $\mathbb{P}_{R_{S}}^{N} \rightarrow \mathbb{P}_{R_{S}}^{N}$ of $R_{S^{-}}$schemes.

Let $\mathcal{P}$ be an unweighted portrait. According to the definition in [56], a map-and-portrait pair $(f, \boldsymbol{P}) \in \operatorname{End}_{d}^{N}[\mathcal{P} \mid 0](K)$ has good reduction outside of $S$ if $\langle f, \boldsymbol{P}\rangle$ is in the image of the natural map

$$
\operatorname{End}_{d}^{N}[\mathcal{P} \mid 0]\left(R_{S}\right) \longrightarrow \mathcal{M}_{d}^{N}[\mathcal{P} \mid 0](K) .
$$

What this means, essentially, is that after a change of coordinates, the map $f$ is defined over $K$, has good reduction outside $S$, and the points $\boldsymbol{P}=\left(P_{v}\right)_{v \in \mathcal{V}}$ form a $\operatorname{Gal}(\bar{K} / K)$-invariant set whose elements remain distinct modulo $\mathfrak{P}$ for all primes $\mathfrak{P}$ lying over primes $\mathfrak{p} \notin S$. 
A stricter version of good reduction asks that we start with a pair $(f, \boldsymbol{P}) \in \operatorname{End}_{d}^{N}[\mathcal{P} \mid 1](K)$, i.e., we insist that the individual points $\left(P_{v}\right)_{v \in \mathcal{V}}$ satisfy $P_{v} \in \mathbb{P}^{N}(K)$; cf. [56].

In general, there are infinitely many $\mathrm{PGL}_{N+1}\left(R_{S}\right)$-conjugacy classes of maps $f \in \operatorname{End}_{d}^{N}(K)$ having good reduction outside $S$. For example, every monic polynomial in $R_{S}[x]$ gives an endomorphism of $\mathbb{P}^{1}$ having good reduction outside $S$. On the other hand, if we add enough level structure, i.e., add a portrait with enough vertices, then at least for $\mathbb{P}^{1}$ there are only finitely many elements of $\operatorname{End}_{d}^{1}[\mathcal{P} \mid 1](K) / \mathrm{PGL}_{2}\left(R_{S}\right)$ having good reduction; see [56, Theorem 2(a)]. In any case, one might expect the maps having good reduction outside $S$ to be reasonably rare. In 56. we defined the Shafarevich dimension as a way to measure the abundance or scarcity of good reduction maps with portraits.

Definition 18.2. Let $N \geq 1$, let $d \geq 2$, let $\mathcal{P}$ be an unweighted portrait, and let $\mathcal{A} \subset \operatorname{Aut}(\mathcal{P})$. The $(N, d)$-Shafarevich dimension of $(\mathcal{P}, \mathcal{A})$ is

$$
\begin{aligned}
& \operatorname{ShafDim}_{d}^{N}[\mathcal{P} \mid \mathcal{A}] \\
& :=\underset{\substack{K \text { a number field } \\
S \text { a finite set of places }}}{\operatorname{dim}} \overline{\operatorname{Image}\left(\operatorname{End}_{d}^{N}[\mathcal{P} \mid \mathcal{A}]\left(R_{S}\right) \longrightarrow \mathcal{M}_{d}^{N}(K)\right)},
\end{aligned}
$$

where the overscore indicates Zariski closure. If $\mathcal{A}=\{1\}$, we may omit it from the notation, and if $\mathcal{P}=\emptyset$, we write simply $\operatorname{ShafDim}_{d}^{N}$.

For example, it is proven in [56, Proposition 12] that

$$
\operatorname{ShafDim}_{2}^{1}=2, \quad \text { and that } \operatorname{ShafDim}_{d}^{1} \geq d+1 \text { for all } d \geq 3 \text {. }
$$

Since $\operatorname{dim} \mathcal{M}_{d}^{1}=2 d-2$, these estimates are optimal for $d=2$ and $d=3$. It would be interesting to know if they are optimal for larger values of $d$.

For $N=1$ and weighted portraits, there are three versions of good reduction described in [56]. Roughly speaking, in addition to requiring that $f$ have good reduction and that the points in $\left(P_{v}\right)_{v \in \mathcal{V}}$ remain distinct for primes not in $S$, we want to impose a condition relating the ramification indices of $f$ at the marked points to the weight function of the portrait. Thus we can require that the ramification indices of $f$ exceed the weights specified by $\epsilon$, or we can require that the ramification indices of $f$ exactly equal the weights specified by $\epsilon$, or we can require that the ramification indices remain exactly equal to the weights even after reduction modulo every prime not in $S$. These cases are assigned symbols [56], which we summarize by:

--good reduction: $e_{f}\left(P_{v}\right) \geq \epsilon\left(P_{v}\right)$ for all $P_{v} \in \mathcal{V}^{\circ}$. 
o-good reduction : $e_{f}\left(P_{v}\right)=\epsilon\left(P_{v}\right)$ for all $P_{v} \in \mathcal{V}^{\circ}$.



We now translate these definitions into moduli-theoretic terms. We fix a weighted portrait $\mathcal{P}$, and we let $(f, P) \in \operatorname{End}_{d}^{1}[\mathcal{P} \mid \mathcal{A}](K)$. Then, after an appropriate change of coordinates for $(f, P)$ :

$(f, P)$ has $\bullet$-good reduction $\Longleftrightarrow \quad(f, P) \in \operatorname{End}_{d}^{1}[\mathcal{P} \mid \mathcal{A}]\left(R_{S}\right)$.

$(f, P)$ has o-good reduction $\Longleftrightarrow(f, P) \in \operatorname{End}_{d}^{1}[\mathcal{P} \mid \mathcal{A}]^{\circ}\left(R_{S}\right)$.

$(f, P)$ has $\star$-good reduction $\Longleftrightarrow \widehat{(f, P)} \in \operatorname{End}_{d}^{1}[\mathcal{P} \mid \mathcal{A}]^{\circ}\left(\overline{\mathbb{F}}_{\mathfrak{p}}\right) \forall \mathfrak{p} \notin S$.

For each type of good reduction, we define an associated Shafarevich dimension. Thus following the notation set in [56], for each symbol $x \in\{\bullet, \circ, \star\}$, we let

$$
\begin{aligned}
& \mathcal{G R}_{d}^{1}[\mathcal{P} \mid \mathcal{A}]^{x}(K, S) \\
&:=\left\{(f, P) \in \operatorname{End}_{d}^{1}[\mathcal{P} \mid \mathcal{A}](K): \begin{array}{l}
(f, P) \text { has } x \text {-good } \\
\text { reduction outside } S
\end{array}\right\},
\end{aligned}
$$

and then we set

$$
\begin{aligned}
& \operatorname{ShafDim}_{d}^{1}[\mathcal{P} \mid \mathcal{A}]^{x} \\
& :=\sup _{S}^{K \text { a number field }} \operatorname{dim} \overline{\operatorname{Image}\left(\mathcal{G} \mathcal{R}_{d}^{1}[\mathcal{P} \mid \mathcal{A}]^{x}(K, S) \longrightarrow \mathcal{M}_{d}^{N}(K)\right)} .
\end{aligned}
$$

We note that these dimensions satisfy [56, Proposition 17]

$$
\operatorname{ShafDim}_{d}^{1}[\mathcal{P} \mid \mathcal{A}]^{\star} \leq \operatorname{ShafDim}_{d}^{1}[\mathcal{P} \mid \mathcal{A}]^{\circ} \leq \operatorname{ShafDim}_{d}^{1}[\mathcal{P} \mid \mathcal{A}]^{\bullet} \leq 2 d-2
$$

It is proven in [56, Theorem 1] that if $\mathcal{P}=\left(\mathcal{V}^{\circ}, \mathcal{V}, \Phi, \epsilon\right)$ is a weighted portrait with total weight

$$
\operatorname{wt}(\mathcal{P}):=\sum_{v \in \mathcal{V}^{\circ}} \epsilon(v) \geq 2 d+1
$$

then $\operatorname{ShafDim}_{d}^{1}[\mathcal{P}]^{\bullet}=0$, but that there are always portraits with $\operatorname{wt}(\mathcal{P})=2 d$ satisfying $\operatorname{ShafDim}_{d}^{1}[\mathcal{P}]^{\bullet} \geq 1$. The paper [56] contains an exhaustive analysis of the 35 portraits $\mathcal{P}$ satisfying $\operatorname{wt}(\mathcal{P}) \leq 4$ and $\mathcal{M}_{2}^{1}[\mathcal{P}] \neq \emptyset$, including a calculation of all three Shafarevich dimensions. For example, it is shown that there are:

10 portraits $\mathcal{P}$ with $\operatorname{wt}(\mathcal{P})=4$ and $\operatorname{ShafDim}_{2}^{1}[\mathcal{P}]^{\bullet}=1$;

12 portraits $\mathcal{P}$ with $w t(\mathcal{P})=4$ and $\operatorname{ShafDim}_{2}^{1}[\mathcal{P}]^{\bullet}=0$;

6 portraits $\mathcal{P}$ with wt $(\mathcal{P})=4$ and $\operatorname{ShafDim}_{2}^{1}[\mathcal{P}]^{\star}=1$. 


\section{Multiplier Systems for Periodic Portraits}

A fundamental tool in studying the dynamics of rational maps on $\mathbb{P}^{1}$ is the use of multipliers at periodic points, since multipliers are $\mathrm{PGL}_{2^{-}}$ invariants. Over a valued field such as $\mathbb{C}$ or $\mathbb{C}_{p}$, the magnitude of the multiplier helps to determine the short-term local behavior of the map. Algebraically, appropriate symmetric functions of the multipliers give $\mathrm{PGL}_{2}$-invariant regular functions on $\operatorname{End}_{d}^{1}$, and hence descend to functions on $\mathcal{M}_{d}^{1}$. Further, one can decompose $\operatorname{End}_{d}^{1}$ or $\mathcal{M}_{d}^{1}$ into a (nicely) varying union of slices by specifying one or more multiplier values. Thus one might look at

$$
\operatorname{Per}_{d, p}(\lambda):=\left\{f \in \mathcal{M}_{d}^{1}: \begin{array}{l}
f \text { has a point } P \text { of (formal) } \\
\text { period } p \text { and multiplier } \lambda
\end{array}\right\},
$$

or more generally one can specify the multipliers for a list of periodic points. See for example [46, Appendix D] for a description of the geometry of the curves $\operatorname{Per}_{2, p}(\lambda)$ in $\mathcal{M}_{2}^{1} \cong \mathbb{A}^{2}$, and [40, 41] for the use of these spaces in studying the stability loci of families of maps on $\mathbb{P}^{1}$.

The classical definition of the multiplier of a rational function $f(z)$ at a $p$-periodic point $\alpha$ is as the derivative $\lambda_{f}(\alpha):=\left(f^{p}\right)^{\prime}(\alpha)$ of the $p$ th iterate of $f$ at $\alpha$, but a more intrinsic definition, which also obviates the need to treat $\infty$ separately, is to note that $f^{p}$ induces a linear transformation

$$
D f_{\alpha}^{p}: \mathcal{T}_{\alpha} \longrightarrow \mathcal{T}_{\alpha}
$$

of the tangent space $\mathcal{T}_{\alpha}$ of $\mathbb{P}^{1}$ at $\alpha$. The space $\mathcal{T}_{\alpha}$ has dimension 1 , and $D f_{\alpha}^{p}$ is multiplication by the scalar $\lambda_{f}(\alpha)$. This characterization also makes it clear that the multiplier is $\mathrm{PGL}_{2}$-invariant, i.e., we have $\lambda_{f} \varphi\left(\varphi^{-1}(\alpha)\right)=\lambda_{f}(\alpha)$. Our goal in this section is to generalize this construction to endomorphisms of $\mathbb{P}^{N}$ for all $N \geq 1$.

19.1. The Multiplier Map for a Single $p$-Cycle. We start with the case of a single cycle, and we let $\mathcal{C}_{p}$ denote the portrait consisting of a $p$-cycle, say

$$
\mathcal{C}_{p}:=\left(\mathbb{Z} / p \mathbb{Z}, \Phi_{p}\right) \quad \text { with } \quad \Phi_{p}(i)=i+1 \bmod p .
$$

A geometric point of $\operatorname{End}_{d}^{N}\left[\mathcal{C}_{p}\right]$ is a map $f$ and a $p$-tuple of distinct points that are cyclically permuted by $f$. Our general construction describes $\operatorname{End}_{d}^{N}\left[\mathcal{C}_{p}\right]$ and $\overline{\operatorname{End}}_{d}^{N}\left[\mathcal{C}_{p}\right]$ as subschemes of $\operatorname{End}_{d}^{N} \times\left(\mathbb{P}^{N}\right)^{p}$, but for the purposes of this section, it is convenient to observe that they may be naturally described as subschemes of $\operatorname{End}_{d}^{N} \times \mathbb{P}^{N}$ by simply projecting onto the first copy of $\mathbb{P}^{N}$. Alternatively, we can start with 
a scheme

$$
\widehat{\operatorname{End}}_{d}^{N}\left[\mathcal{C}_{p}\right]:=\left\{(f, P) \in \operatorname{End}_{d}^{N} \times \mathbb{P}^{N}: f^{p}(P)=P\right\}
$$

that is "too large" and remove the parts that we don't want. Thus

$$
\operatorname{End}_{d}^{N}\left[\mathcal{C}_{p}\right] \cong \widehat{\operatorname{End}}_{d}^{N}\left[\mathcal{C}_{p}\right] \backslash \bigcup_{\substack{q \mid p \\ q<p}} \widehat{\operatorname{End}}_{d}^{N}\left[\mathcal{C}_{q}\right]
$$

We note that $\overline{\operatorname{End}}_{d}^{N}\left[\mathcal{C}_{p}\right]$ is the closure of $\operatorname{End}_{d}^{N}\left[\mathcal{C}_{p}\right]$ in $\widehat{\operatorname{End}}_{d}^{N}\left[\mathcal{C}_{p}\right]$, and that there is a natural action of $\mathbb{Z} / p \mathbb{Z}$ on each of these spaces via

$$
\mathbb{Z} / p \mathbb{Z} \longrightarrow \operatorname{Aut}\left(\widehat{\operatorname{End}}_{d}^{N}\left[\mathcal{C}_{p}\right]\right), \quad j \longmapsto\left((f, P) \mapsto\left(f, f^{j}(P)\right)\right) .
$$

With this construction, a geometric point of $\operatorname{End}_{d}^{N}\left[\mathcal{C}_{p}\right]$ consists of a map $f$ and a point $P$ whose orbit is a cycle of exact period $p$. In particular, the point $P$ is fixed by the $p$ th iterate $f^{p}$, so we get an induced map on the tangent space of $\mathbb{P}^{N}$ at $P$,

$$
D f_{P}^{p}: \mathcal{T}_{P} \longrightarrow \mathcal{T}_{P}
$$

We write the characteristic polynomial of $D f_{P}^{p}$ as

$$
\operatorname{det}\left(X-D f_{P}^{p}\right)=\sum_{i=0}^{N}(-1)^{i} \Lambda_{i}\left(f^{p}, P\right) X^{N-i}
$$

so $\Lambda_{i}\left(f^{p}, P\right)$ is the $i$ th symmetric polynomial of the eigenvalues of the linear transformation $D f_{P}^{p}$. These eigenvalues are typically called the multipliers of $f$ at $P$.

Each coefficient of the characteristic polynomial defines a regular function

$$
\operatorname{End}_{d}^{N}\left[\mathcal{C}_{p}\right] \longrightarrow \mathbb{A}^{1}, \quad(f, P) \longmapsto \Lambda_{i}\left(f^{p}, P\right) .
$$

We call these $\Lambda_{i}\left(f^{p}, P\right)$ the multiplier coefficients of $f$ at $P$, and we fit them together to define the multiplier map

$$
\mu_{p}: \operatorname{End}_{d}^{N}\left[\mathcal{C}_{p}\right] \longrightarrow \mathbb{A}^{N}, \quad(f, P) \longmapsto\left(\Lambda_{1}\left(f^{p}, P\right), \ldots, \Lambda_{N}\left(f^{p}, P\right)\right) .
$$

The fact that the characteristic polynomial is invariant for the action of $\mathrm{PGL}_{N+1}$, i.e.,

$$
\Lambda_{i}\left(\varphi^{-1} \circ f^{p} \circ \varphi, \varphi^{-1}(P)\right)=\Lambda_{i}\left(f^{p}, P\right),
$$

shows that the multiplier map descends to give a multiplier map

$$
\mu_{p}: \mathcal{M}_{d}^{N}\left[\mathcal{C}_{p}\right] \rightarrow \mathbb{A}^{N}
$$


on the portrait moduli space. Further, since the multipliers are invariant for the action (81), i.e., we have $\Lambda_{i}\left(f^{p}, P\right)=\Lambda_{i}\left(f^{p}, f^{j}(P)\right)$ for all $j \in \mathbb{Z} / p \mathbb{Z}$, the multiplier map descends to a map on the quotient space

$$
\mu_{p}: \mathcal{M}_{d}^{N}\left[\mathcal{C}_{p} \mid \mathbb{Z} / p \mathbb{Z}\right] \rightarrow \mathbb{A}^{N}
$$

We claim that the multiplier map extends to $\overline{\operatorname{End}}_{d}^{N}\left[\mathcal{C}_{p}\right]$ and $\overline{\mathcal{M}}_{d}^{N}\left[\mathcal{C}_{p}\right]$, and more generally, it extends to spaces attached to disjoint unions of cyclic portraits. The easiest way to see this is to define the multiplier map on a generalization of the larger scheme (80) that we used earlier.

\subsection{The Multiplier Map for Cycles of Length $\boldsymbol{p}_{1}, \ldots, \boldsymbol{p}_{n}$.}

Let $f=\left[f_{0}, \ldots, f_{N}\right]$ be a generic element of $\operatorname{End}_{d}^{N}$, i.e., the coefficients of $f_{0}, \ldots, f_{N}$ are indeterminates $a_{0}(\boldsymbol{e}), \ldots, a_{N}(\boldsymbol{e})$ as in (2). We write the $p$ th iterate of $f$ as a polynomial of degree $d^{p}$ with coefficients that are polynomials in the $a_{\rho}(\boldsymbol{e})$, say

$$
f^{p}(\boldsymbol{x})=\left[F_{0}^{(p)}(\boldsymbol{x}), \ldots, F_{N}^{(p)}(\boldsymbol{x})\right] .
$$

As in the proof of Theorem 8.1, we want to define various subschemes of $\operatorname{End}_{d}^{N} \times\left(\mathbb{P}^{N}\right)^{n}$, so for each $1 \leq \nu \leq n$, we let

$$
\boldsymbol{U}_{\nu}:=\left[U_{\nu}(0), \ldots, U_{\nu}(N)\right]=\left\{\begin{array}{l}
\text { homogeneous coordinates on } \\
\text { the } \nu \text { th copy of } \mathbb{P}^{N} \text { in }\left(\mathbb{P}^{N}\right)^{n}
\end{array}\right\},
$$

and we let

$$
\boldsymbol{U}:=\left(\boldsymbol{U}_{1}, \ldots, \boldsymbol{U}_{n}\right) .
$$

We also fix an $n$-tuple of periods $\boldsymbol{p}:=\left(p_{1}, \ldots, p_{n}\right)$, and we write

$$
\mathcal{C}_{p}:=\mathcal{C}_{p_{1}} \cup \mathcal{C}_{p_{2}} \cup \cdots \cup \mathcal{C}_{p_{n}}
$$

for the portrait consisting of a disjoint union of periodic cycles of the indicated periods. We then define a large subscheme of $\operatorname{End}_{d}^{N} \times\left(\mathbb{P}^{N}\right)^{n}$, generalizing (80), by

$$
\widehat{\operatorname{End}}_{d}^{N}\left[\mathcal{C}_{\boldsymbol{p}}\right]:=\bigcap_{\nu=1}^{n} \bigcap_{i, j=1}^{N}\left\{(f, \boldsymbol{U}): U_{\nu}(i) F_{j}^{\left(p_{\nu}\right)}\left(\boldsymbol{U}_{\nu}\right)-U_{\nu}(j) F_{i}^{\left(p_{\nu}\right)}\left(\boldsymbol{U}_{\nu}\right)\right\} .
$$

This formula is how we define the structure of $\widehat{\operatorname{End}}_{d}^{N}\left[\mathcal{C}_{p}\right]$ explicitly as a subscheme of the product $\operatorname{End}_{d}^{N} \times\left(\mathbb{P}^{N}\right)^{n}$, but intuitively at the level of geometric points, we see that

$$
\begin{aligned}
\widehat{\operatorname{End}}_{d}^{N}\left[\mathcal{C}_{\boldsymbol{p}}\right] & := \\
& \left\{(f, \boldsymbol{P}) \in \operatorname{End}_{d}^{N} \times\left(\mathbb{P}^{N}\right)^{n}: f^{p_{\nu}}\left(P_{\nu}\right)=P_{\nu} \text { for all } 1 \leq \nu \leq n\right\} .
\end{aligned}
$$

We say that the scheme $\widehat{\operatorname{End}}_{d}^{N}\left[\mathcal{C}_{\boldsymbol{p}}\right]$ is "large" because it tends to include extra components beyond the closure of $\operatorname{End}_{d}^{N}\left[\mathcal{C}_{p}\right]$. This is due to the 
fact that we do not require the orbits of the $P_{\nu}$ to be disjoint, nor do we require individual $P_{\nu}$ to have exact or formal period $p_{\nu}$.

For each $0<i \leq N$ and each $1 \leq \nu \leq n$, the multiplier coefficient $\Lambda_{i}\left(f^{p_{\nu}}, P_{\nu}\right)$ gives a regular function

$$
\widehat{\operatorname{End}}_{d}^{N}\left[\mathcal{C}_{\boldsymbol{p}}\right] \longrightarrow \mathbb{A}^{1}, \quad(f, \boldsymbol{P}) \longmapsto \Lambda_{i}\left(f^{p_{\nu}}, P_{\nu}\right) .
$$

Fitting these together, we obtain a multiplier map

$$
\mu_{\boldsymbol{p}}: \widehat{\operatorname{End}}_{d}^{N}\left[\mathcal{C}_{\boldsymbol{p}}\right] \longrightarrow \mathbb{A}^{N n}, \quad(f, \boldsymbol{P}) \longmapsto\left(\Lambda_{i}\left(f^{p_{\nu}}, P_{\nu}\right)\right)_{\substack{0<i \leq N \\ 1 \leq \nu \leq n}}
$$

N.B. The map $\mu_{p}$ is a morphism on this large closed subscheme of $\operatorname{End}_{d}^{N} \times\left(\mathbb{P}^{N}\right)^{n}$. By restriction, we obtain a morphism on any subscheme of this large scheme, so in particular we get multiplier maps

$$
\mu_{\boldsymbol{p}}: \operatorname{End}_{d}^{N}\left[\mathcal{C}_{\boldsymbol{p}}\right] \longrightarrow \mathbb{A}^{N n} \text { and } \mu_{\boldsymbol{p}}: \overline{\operatorname{End}}_{d}^{N}\left[\mathcal{C}_{\boldsymbol{p}}\right] \longrightarrow \mathbb{A}^{N n} \text {. }
$$

But (82) says that these maps are invariant for the action of $\mathrm{PGL}_{N+1}$ on $\operatorname{End}_{d}^{N}\left[\mathcal{C}_{p}\right]$, hence they descend and give well-defined maps as in the next definition.

Definition 19.1. Let $\boldsymbol{p}=\left(p_{1}, \ldots, p_{n}\right)$. The multiplier maps (morphisms) on the associated portrait moduli spaces are denoted

$$
\mu_{\boldsymbol{p}}: \mathcal{M}_{d}^{N}\left[\mathcal{C}_{\boldsymbol{p}}\right] \longrightarrow \mathbb{A}^{N n} \text { and } \mu_{\boldsymbol{p}}: \overline{\mathcal{M}}_{d}^{N}\left[\mathcal{C}_{\boldsymbol{p}}\right] \longrightarrow \mathbb{A}^{N n}
$$

Using these multiplier maps allows us to generalize the classical decomposition (79) of $\mathcal{M}_{d}^{1}$ in a variety of ways, for example, by taking inverse images of points in $\mathbb{A}^{N n}$. More generally, we can fiber $\mathbb{A}^{N n}$ using $k$-dimensional linear subspaces, leading to a decomposition of $\overline{\mathcal{M}}_{d}^{N}$ as a union

$$
\overline{\mathcal{M}}_{d}^{N}=\pi\left(\bigcup_{L \in \mathrm{Gr}_{k}^{N n}} \mu_{\boldsymbol{p}}^{-1}(L)\right),
$$

where $\pi: \overline{\mathcal{M}}_{d}^{N}\left[\mathcal{C}_{\boldsymbol{p}}\right] \rightarrow \overline{\mathcal{M}}_{d}^{N}$ denotes the natural projection map and $\mathrm{Gr}_{k}^{N n}$ is the Grassmanian parameterizing $k$-dimensional linear subspaces of $\mathbb{A}^{N n}$.

19.3. The Multiplier Map for Full $p$-Cycle Structure. For this subsection we again assume:

$$
\text { All varieties are defined over a field of characteristic } 0 \text {. }
$$

In order to extract the maximum amount of information from periodic points of a map $f$, we should use all of the periodic points of a given period. For integers $N \geq 1, d \geq 2$, and $p \geq 1$, we recall that a map $f \in \operatorname{End}_{d}^{N}$ has exactly $\nu_{d}^{N}(p)$ geometric periodic points of formal period $p$, counted with appropriate multiplicities, where $\nu_{d}^{N}(p)$ is given 
by (61) in Definition [14.1; see [29, Theorems 4.3 and 4.17]. Further, Lemma 14.4 says that a generic map $f \in \operatorname{End}_{d}^{N}$ has exactly this many geometric points of exact period $p$. To ease notation, for the remainder of this section we let

$$
\bar{\nu}(p):=\frac{1}{p} \nu_{d}^{N}(p)=\frac{1}{p} \sum_{k \mid p}\left(\mu(p / k) \sum_{j=0}^{N} d^{j k}\right)
$$

denote the number of geometric $p$-cycles of a generic $f \in \operatorname{End}_{d}^{N}$.

By a slight abuse of notation, we let

$$
\boldsymbol{p}(N, d):=(\underbrace{p, p, p, \ldots, p}_{\bar{\nu}(p) \text { copies of } p})
$$

so the portrait $\mathcal{C}_{\boldsymbol{p}(N, d)}$ is given by

$$
\mathcal{C}_{\boldsymbol{p}(N, d)}=\text { a disjoint union of } \bar{\nu}(p) \text { periodic cycles of period } p .
$$

The $(\mathrm{f}) \Longrightarrow(\mathrm{c})$ implication of Theorem 14.2 tells us that

$$
\overline{\operatorname{End}}_{d}^{N}\left[\mathcal{C}_{\boldsymbol{p}(N, d)}\right] \rightarrow \operatorname{End}_{d}^{N}
$$

is a finite surjective morphism. (This is where we use our characteristic 0 assumption, since it is required for Theorem 14.2.)

We let

$$
\mathcal{A}_{N, d, p}=\operatorname{Aut}\left(\mathcal{C}_{\boldsymbol{p}(N, d)}\right) \cong \mathcal{S}_{\bar{\nu}(p)} \gamma(\mathbb{Z} / p \mathbb{Z}) \cong \mathcal{S}_{\bar{\nu}(p)} \rtimes(\mathbb{Z} / p \mathbb{Z})^{\bar{\nu}(p)}
$$

be the full automorphism group of our disjoint union of $p$-cycles. It is a wreath product consisting of permutations and rotations of the cycles.

As above, there are multiplier maps (84) associated to the full $p$ period portraits $\mathcal{C}_{\boldsymbol{p}(N, d)}$. The pure cycle rotations (81) in $\mathcal{A}_{N, d, p}$ have no effect on the multipliers, so leave the multiplier map invariant, while the permutation part of $\mathcal{A}_{N, d, p}$ corresponds to permuting the various copies of $\mathbb{A}^{N}$ in the images of the multiplier maps (83). Hence we obtain multiplier maps (which by abuse of notation we continue to call $\mu_{\boldsymbol{p}(N, d)}$ )

$$
\begin{aligned}
& \mu_{\boldsymbol{p}(N, d)}: \mathcal{M}_{d}^{N}\left[\mathcal{C}_{\boldsymbol{p}} \mid \mathcal{A}_{N, d, p}\right] \longrightarrow\left(\mathbb{A}^{\bar{\nu}(p)}\right)^{N} / \mathcal{S}_{\bar{\nu}(p)}, \\
& \mu_{\boldsymbol{p}(N, d)}: \overline{\mathcal{M}}_{d}^{N}\left[\mathcal{C}_{\boldsymbol{p}} \mid \mathcal{A}_{N, d, p}\right] \longrightarrow\left(\mathbb{A}^{\bar{\nu}(p)}\right)^{N} / \mathcal{S}_{\bar{\nu}(p)},
\end{aligned}
$$

where $\mathcal{S}_{\bar{\nu}(p)}$ acts diagonally on $\left(\mathbb{A}^{\bar{\nu}(p)}\right)^{N}$.

We next observe that the map $\mathcal{M}_{d}^{N}\left[\mathcal{C}_{p}\right] \rightarrow \mathcal{M}_{d}^{N}$ factors through $\mathcal{M}_{d}^{N}\left[\mathcal{C}_{p} \mid \mathcal{A}_{N, d, p}\right]$, since the action of $\mathcal{A}_{N, d, p}$ on $\left(f, P_{1}, \ldots, P_{\bar{\nu}(p)}\right)$ leaves $f$ invariant. On the other hand, both of the maps

$$
\mathcal{M}_{d}^{N}\left[\mathcal{C}_{p}\right] \rightarrow \mathcal{M}_{d}^{N}\left[\mathcal{C}_{p} \mid \mathcal{A}_{N, d, p}\right] \quad \text { and } \quad \mathcal{M}_{d}^{N}\left[\mathcal{C}_{p}\right] \rightarrow \mathcal{M}_{d}^{N}
$$


have generic degree equal to $\# \mathcal{A}_{N, d, p}=\bar{\nu}(p) ! \cdot p^{\bar{\nu}(p)}$. Hence the map $\mathcal{M}_{d}^{N}\left[\mathcal{C}_{p} \mid \mathcal{A}_{N, d, p}\right] \rightarrow \mathcal{M}_{d}^{N}$ is an isomorphism, so (85) may be viewed as a multiplier map

$$
\mu_{\boldsymbol{p}(N, d)}: \mathcal{M}_{d}^{N} \longrightarrow\left(\mathbb{A}^{\bar{\nu}(p)}\right)^{N} / \mathcal{S}_{\bar{\nu}(p)} .
$$

Remark 19.2. When $N \geq 2$, there are some advantages to taking a further quotient in which we allow $N$ copies of $\mathcal{S}_{\bar{\nu}(p)}$ to act independently on the $N$ copies of $\mathbb{A}^{\bar{\nu}(p)}$, and then use the general fact that $\mathbb{A}^{\nu} / \mathcal{S}_{\nu} \cong \mathbb{A}^{\nu}$ via the elementary symmetric functions. This gives a "collapsed" multiplier map

$$
\mu_{\boldsymbol{p}(N, d)}^{\prime}: \mathcal{M}_{d}^{N} \stackrel{\mu_{\boldsymbol{d}(N, d)}}{\longrightarrow}\left(\mathbb{A}^{\bar{\nu}(p)}\right)^{N} / \mathcal{S}_{\bar{\nu}(p)}, \longrightarrow\left(\mathbb{A}^{\bar{\nu}(p)} / \mathcal{S}_{\bar{\nu}(p)}\right)^{N} \stackrel{\sim}{\longrightarrow} \mathbb{A}^{\bar{\nu}(p) N} .
$$

We note that these maps coincide for $N=1$, i.e., $\mu_{\boldsymbol{p}(1, d)}^{\prime}=\mu_{\boldsymbol{p}(1, d)}$.

A famous theorem of McMullen says that for $N=1$ and in characteristic 0, sufficiently many multipliers (almost) determine the map $\mathrm{PGL}_{2}$-conjugacy class of the map $f$. In our notation, McMullen's theorem says the following.

Theorem 19.3 (McMullen [44]). For every $d \geq 2$ there is a $\kappa(d)$ with the property that the map

$$
\prod_{p \leq \kappa(d)} \mu_{\boldsymbol{p}(N, d)}: \mathcal{M}_{d}^{1}(\mathbb{C}) \longrightarrow \prod_{p \leq \kappa(d)} \mathbb{C}^{\bar{\nu}(p)}
$$

is quasi-finite away from the locus of flexible Lattès maps.

This naturally leads to two questions, as described in [59, Question 2.43].

Question 19.4. For $N \geq 2$ and $d \geq 2$, is there a $\kappa(N, d)$ such that the map

$$
\prod_{p \leq \kappa(N, d)} \mu_{\boldsymbol{p}(N, d)}^{\prime}: \mathcal{M}_{d}^{N}(\mathbb{C}) \longrightarrow \prod_{p \leq \kappa(N, d)} \mathbb{C}^{\bar{\nu}(p) N}
$$

is quasi-finite on a non-empty Zariski open subset? If so, what does the complement look like?

Question 19.5 (Poonen). For $N \geq 1$ and $d \geq 2$, is there a $\kappa(N, d)$ such that the map

$$
\prod_{p \leq \kappa(N, d)} \mu_{\boldsymbol{p}(N, d)}: \mathcal{M}_{d}^{N}(\mathbb{C}) \longrightarrow \prod_{p \leq \kappa(N, d)}\left(\mathbb{C}^{\bar{\nu}(p)}\right)^{N} / \mathcal{S}_{\bar{\nu}(p)}
$$

is one-to-one on a non-empty Zariski open subset of $\mathcal{M}_{d}^{N}(\mathbb{C})$ ? Note that this question is open even for $N=1$. 
19.4. Fixed Point Multiplier Relations. In this section we consider an endomorphism $f: \mathbb{P}^{N} \rightarrow \mathbb{P}^{N}$ of degree $d \geq 2$ and fixed points $P \in \operatorname{Fix}(f)$. We factor the characteristic polynomial of the linear map $D f_{P}: \mathcal{T}_{P} \rightarrow \mathcal{T}_{P}$ as

$$
\operatorname{det}\left(X-D f_{P}\right)=\sum_{i=0}^{N}(-1)^{i} \Lambda_{i}(f, P) X^{N-i}=\prod_{j=1}^{N}\left(X-\lambda_{j}(f, P)\right),
$$

so $\lambda_{1}(f, P), \ldots, \lambda_{N}(f, P)$ are the multipliers of $f$ at $P$, with appropriate multiplicities. We say that $P$ is a simple fixed point if none of its multipliers is equal to 1 .

When $N=1$, each fixed point $P$ of $f$ has a single multiplier $\lambda(P)$, and assuming that the fixed points are simple, their multipliers satisfy the following famous classical relation 24

$$
\sum_{P \in \operatorname{Fix}(f)} \frac{1}{1-\lambda(f, P)}=1
$$

Writing (88) as a symmetric function of the $\lambda(f, P)$, we see that it defines a hypersurface containing the image of

$$
\mu_{\mathbf{1}(1, d)}: \mathcal{M}_{d}^{1} \longrightarrow \mathbb{A}^{d+1}
$$

(Note that an $f \in \operatorname{End}_{d}^{1}$ has $d+1$ fixed points.) For example, when $d=$ 2 the relation (88) says that the image lies in a hyperplane, and indeed, in this case Milnor proved that $\mu_{\mathbf{1}(1,2)}$ gives an isomorphism $\mathcal{M}_{2}^{1} \rightarrow \mathbb{A}^{2}$; cf. Example 13.2.

The classical fixed point multiplier relation (88) for $\mathbb{P}^{1}$ has been generalized by Ueda to endomorphisms of $\mathbb{P}^{N}$.

Theorem 19.6. (Ueda 60]) Let $f \in \operatorname{End}_{d}^{N}$, and assume that all of the fixed points of $f$ are simple. Then

$$
\sum_{P \in \operatorname{Fix}(f)} \frac{\operatorname{det}\left(X-D f_{P}\right)}{\operatorname{det}\left(I-D f_{P}\right)}=\frac{X^{N+1}-d^{N+1}}{X-d} .
$$

Equivalently, for each $0 \leq k \leq N$, we have

$$
\sum_{P \in \operatorname{Fix}(f)} \frac{\Lambda_{k}(f, P)}{\operatorname{det}\left(I-D f_{P}\right)}=(-d)^{k}
$$

\footnotetext{
${ }^{24}$ In his 1919 Mémoire [33, Section 9], Julia says that this relation is "bien connue."
} 
Remark 19.7. Ueda's formula (89) yields many interesting relations. For example, differentiating (89) with respect to $X$ and then setting $X=1$ yields

$$
\sum_{P \in \operatorname{Fix}(f)} \sum_{j=1}^{N} \frac{1}{1-\lambda_{j}(f, P)}=d^{N-1}+2 d^{N-2}+\cdots+(N-1) d+N
$$

which is a natural generalization of the classical 1-dimensional result (88).

Substituting

$$
\operatorname{det}\left(I-D f_{P}\right)=\sum(-1)^{i} \Lambda_{i}(f, P)
$$

into Ueda's relation (90) and clearing the denominators, for each $0 \leq$ $k \leq N$ we obtain a polynomial relation among the collection of multipliers

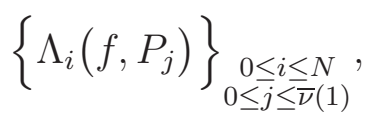

where $P_{1}, \ldots, P_{\bar{\nu}(1)}$ are the fixed points of $f$. This relation is invariant under permutation of the points in $\operatorname{Fix}(f)$, so it descends to define a hypersurface $U_{k}$ having the property that the image of the fixed-point multiplier map

$$
\mu_{\mathbf{1}(N, d)}: \mathcal{M}_{d}^{N} \longrightarrow\left(\mathbb{A}^{\bar{\nu}(1)}\right)^{N} / \mathcal{S}_{\bar{\nu}(1)}
$$

lies in $U_{k} 25$ We call $U_{k}$ a Ueda fixed-point hypersurface, and we observe that these hypersurfaces are defined over $\mathbb{Q}$.

As noted in (87), we can collapse the image of the multiplier map by taking a further quotient. This corresponds to permuting the elements of the sets $\left\{\Lambda_{i}(P)\right\}_{P \in \operatorname{Fix}(f)}$ independently for each $0 \leq i \leq N$, which is somewhat unnatural, since it does not come from a natural action on $\mathcal{M}_{d}^{N}\left[\mathcal{C}_{\mathbf{1}}\right]$. On the other hand, it leads to a simpler target space, so could be useful. In any case, we push forward $U_{k}$ to obtain the collapsed Ueda fixed-point hypersurfaces

$$
U_{k}^{\prime}=\operatorname{Image}\left(U_{k} \subset\left(\mathbb{A}^{\bar{\nu}(1)}\right)^{N} / \mathcal{S}_{\bar{\nu}(1)} \longrightarrow\left(\mathbb{A}^{\bar{\nu}(1)} / \mathcal{S}_{\bar{\nu}(1)}\right)^{N} \cong \mathbb{A}^{\bar{\nu}(1) N}\right) .
$$

\footnotetext{
${ }^{25}$ We only know, a priori, that the maps $f \in \mathcal{M}_{d}^{N}$ with simple fixed points map to points in the hypersurface $U_{k}$, but the maps with simple fixed points form a non-empty Zariski open subset, so the entire image of $\mathcal{M}_{d}^{N}$ must lie in the closed hypersurface $U_{k}$.
} 
At most $N$ of the $N+1$ hypersurfaces $U_{0}, \ldots, U_{N}$ can be independent, since

$$
\begin{gathered}
\sum_{k=0}^{N} \overbrace{\left(\sum_{P \in \operatorname{Fix}(f)}(-1)^{k} \frac{\Lambda_{k}(f, P)}{\operatorname{det}\left(I-D f_{P}\right)}-d^{k}\right)}^{\text {equation that determines } U_{k}} \\
=\sum_{P \in \operatorname{Fix}(f)} \frac{\sum_{k=0}^{N}(-1)^{k} \Lambda_{k}(f, P)}{\operatorname{det}\left(I-D f_{P}\right)}-\sum_{k=0}^{N} d^{k} \\
=\sum_{P \in \operatorname{Fix}(f)} \frac{\operatorname{det}\left(I-D f_{P}\right)}{\operatorname{det}\left(I-D f_{P}\right)}-\sum_{k=0}^{N} d^{k}=0
\end{gathered}
$$

where for the last equality we have used the fact that \# $\operatorname{Fix}(f)=$ $\left(d^{N+1}-1\right) /(d-1)$. It seems likely that there are no other relations.

Conjecture 19.8. (a) The collapsed Ueda hypersurfaces $U_{0}^{\prime}, \ldots, U_{N}^{\prime}$ are all irreducible.

(b) The intersection of the collapsed Ueda hypersurfaces $U_{0}^{\prime}, \ldots, U_{N}^{\prime}$ is a subvariety of $\mathbb{A}^{\bar{\nu}(1) N}$ of codimension $N$.

(c) Question: Is the intersection $\bigcap U_{k}^{\prime}$ irreducible?

Acknowledgements. The authors would like to thank Dan Abramovich, Eric Bedford, David Eppstein, Noah Giansiracusa, Jeremy Kahn, and Michael Rosen for their helpful advice.

\section{REFERENCES}

[1] Matthieu Arfeux. Compactification and trees of spheres covers. Conform. Geom. Dyn., 21:225-246, 2017.

[2] Ben Bielefeld, Yuval Fisher, and John Hubbard. The classification of critically preperiodic polynomials as dynamical systems. J. Amer. Math. Soc., 5(4):721$762,1992$.

[3] Jérémy Blanc, Jung Kyu Canci, and Noam D. Elkies. Moduli spaces of quadratic rational maps with a marked periodic point of small order. Int. Math. Res. Not. IMRN, (23):12459-12489, 2015.

[4] Wieb Bosma, John Cannon, and Catherine Playoust. The Magma algebra system. I. The user language. J. Symbolic Comput., 24(3-4):235-265, 1997. Computational algebra and number theory (London, 1993).

[5] T. Bousch. Sur quelques problèmes de dynamique holomorphe. $\mathrm{PhD}$ thesis, Université de Paris-Sud, Centre d'Orsay, 1992.

[6] Xavier Buff, A.L. Epstein, and Sarah Koch. Irreducibility and postcritically finite unicritical polynomials, 2018. arXiv.1806.11221. 
[7] Xavier Buff and Tan Lei. The quadratic dynatomic curves are smooth and irreducible. In Frontiers in complex dynamics, volume 51 of Princeton Math. Ser., pages 49-72. Princeton Univ. Press, Princeton, NJ, 2014.

[8] Jung Kyu Canci and Solomon Vishkautsan. Quadratic maps with a periodic critical point of period 2. Int. J. Number Theory, 13(6):1393-1417, 2017.

[9] Kristin Cordwell, Selina Gilbertson, Nicholas Nuechterlein, Kevin M. Pilgrim, and Samantha Pinella. On the classification of critically fixed rational maps. Conform. Geom. Dyn., 19:51-94, 2015.

[10] Laura DeMarco. The moduli space of quadratic rational maps. J. Amer. Math. Soc., 20(2):321-355, 2007.

[11] Igor Dolgachev. Lectures on invariant theory, volume 296 of London Mathematical Society Lecture Note Series. Cambridge University Press, Cambridge, 2003.

[12] Igor V. Dolgachev and Yi Hu. Variation of geometric invariant theory quotients. Inst. Hautes Études Sci. Publ. Math., (87):5-56, 1998. With an appendix by Nicolas Ressayre.

[13] A. Douady and J. H. Hubbard. Étude dynamique des polynômes complexes. Partie I, volume 84 of Publications Mathématiques d'Orsay [Mathematical Publications of Orsay]. Université de Paris-Sud, Département de Mathématiques, Orsay, 1984.

[14] A. Douady and J. H. Hubbard. Étude dynamique des polynômes complexes. Partie II, volume 85 of Publications Mathématiques d'Orsay [Mathematical Publications of Orsay]. Université de Paris-Sud, Département de Mathématiques, Orsay, 1985. With the collaboration of P. Lavaurs, Tan Lei and P. Sentenac.

[15] Adrien Douady and John H. Hubbard. A proof of Thurston's topological characterization of rational functions. Acta Math., 171(2):263-297, 1993.

[16] John R. Doyle. Dynamical modular curves for quadratic polynomial maps. Trans. Amer. Math. Soc., 2018. to appear, https://doi.org/10.1090/tran/7474.

[17] John R. Doyle, Holly Krieger, Andrew Obus, Rachel Pries, Simon Rubinstein-Salzedo, and Lloyd W. West. Reduction of dynatomic curves. Ergodic Theory Dynam. Systems, 2018. to appear, https://doi.org/10.1017/etds.2017.140.

[18] John R. Doyle and Bjorn Poonen. Gonality of dynatomic curves and strong uniform boundedness of preperiodic points, 2017. arXiv:1711.04233.

[19] John R. Doyle and Joseph H. Silverman. A uniform field-of-definition/fieldof-moduli bound for dynamical systems on $\mathbb{P}^{N}$. J. Number Theory, 195:1-22, 2019.

[20] Xander Faber and Andrew Granville. Prime factors of dynamical sequences. J. Reine Angew. Math., 661:189-214, 2011.

[21] Najmuddin Fakhruddin. Questions on self maps of algebraic varieties. J. Ramanujan Math. Soc., 18(2):109-122, 2003.

[22] Najmuddin Fakhruddin. The algebraic dynamics of generic endomorphisms of $\mathbb{P}^{n}$. Algebra Number Theory, 8(3):587-608, 2014.

[23] E. V. Flynn, Bjorn Poonen, and Edward F. Schaefer. Cycles of quadratic polynomials and rational points on a genus-2 curve. Duke Math. J., 90(3):435$463,1997$. 
[24] Yan Gao. Preperiodic dynatomic curves for $z \mapsto z^{d}+c$. Fund. Math., 233(1):3769, 2016.

[25] Yan Gao and YaFei Ou. The dynatomic periodic curves for polynomial $z \mapsto$ $z^{d}+c$ are smooth and irreducible. Sci. China Math., 57(6):1175-1192, 2014.

[26] Dragos Ghioca, Khoa Nguyen, and Thomas J. Tucker. Portraits of preperiodic points for rational maps. Math. Proc. Cambridge Philos. Soc., 159(1):165-186, 2015.

[27] Phillip Griffiths and Joseph Harris. Principles of Algebraic Geometry. Wiley Classics Library. John Wiley \& Sons Inc., New York, 1994. Reprint of the 1978 original.

[28] Robin Hartshorne. Algebraic Geometry, volume 52 of Graduate Texts in Mathematics. Springer-Verlag, New York, 1977.

[29] Benjamin Hutz. Dynatomic cycles for morphisms of projective varieties. New York J. Math., 16:125-159, 2010.

[30] Benjamin Hutz. Effectivity of dynatomic cycles for morphisms of projective varieties using deformation theory. Proc. Amer. Math. Soc., 140(10):3507-3514, 2012.

[31] Benjamin Hutz and Michael Tepper. Multiplier spectra and the moduli space of degree 3 morphisms on $\mathbb{P}^{1}$. JP J. Algebra Number Theory Appl., 29(2):189206, 2013.

[32] Patrick Ingram and Joseph H. Silverman. Primitive divisors in arithmetic dynamics. Math. Proc. Cambridge Philos. Soc., 146(2):289-302, 2009.

[33] G. Julia. Mémoire sur l'itération des fonctions rationelles. Journal de Math. Pures et Appl., 8:47-245, 1918.

[34] David Krumm. A finiteness theorem for specializations of dynatomic polynomials, 2018. arXiv:1805.11152.

[35] David Krumm. Galois groups in a family of dynatomic polynomials. J. Number Theory, 187:469-511, 2018.

[36] E. Lau and D. Schleicher. Internal addresses in the Mandelbrot set and irreducibility of polynomials. Technical Report 1994/19, December 1994.

[37] Genadi Levin, Weixiao Shen, and Sebastian van Strien. Transversality for critical relations of families of rational maps: an elementary proof, 2017. arXiv: 1702.02582

[38] Alon Levy. The space of morphisms on projective space. Acta Arith., 146(1):1331, 2011.

[39] Alon Levy, Michelle Manes, and Bianca Thompson. Uniform bounds for preperiodic points in families of twists. Proc. Amer. Math. Soc., 142(9):3075-3088, 2014.

[40] M. Yu. Lyubich. Some typical properties of the dynamics of rational mappings. Uspekhi Mat. Nauk, 38(5(233)):197-198, 1983.

[41] R. Mañé, P. Sad, and D. Sullivan. On the dynamics of rational maps. Ann. Sci. École Norm. Sup. (4), 16(2):193-217, 1983.

[42] Michelle Manes. Moduli spaces for families of rational maps on $\mathbb{P}^{1}$. J. Number Theory, 129(7):1623-1663, 2009.

[43] Michelle Manes and Joseph H. Silverman. A classification of degree 2 semistable rational maps $\mathbb{P}^{2} \rightarrow \mathbb{P}^{2}$ with large finite dynamical automorphism group, 2016. Annales de la Faculte des Sciences de Toulouse, to appear. arxiv:1607.05772. 
[44] Curtis T. McMullen. Families of rational maps and iterative root-finding algorithms. Ann. of Math. (2), 125(3):467-493, 1987.

[45] James S. Milne. Étale Cohomology, volume 33 of Princeton Mathematical Series. Princeton University Press, Princeton, N.J., 1980.

[46] John Milnor. Geometry and dynamics of quadratic rational maps. Experiment. Math., 2(1):37-83, 1993. With an appendix by the author and Lei Tan.

[47] John Milnor. Dynamics in one complex variable, volume 160 of Annals of Mathematics Studies. Princeton University Press, Princeton, NJ, third edition, 2006.

[48] Patrick Morton. On certain algebraic curves related to polynomial maps. Compositio Math., 103(3):319-350, 1996.

[49] Patrick Morton and Joseph H. Silverman. Rational periodic points of rational functions. Internat. Math. Res. Notices, (2):97-110, 1994.

[50] David Mumford, John Fogarty, and Frances Kirwan. Geometric Invariant Theory, volume 34 of Ergebnisse der Mathematik und ihrer Grenzgebiete (2) [Results in Mathematics and Related Areas (2)]. Springer-Verlag, Berlin, third edition, 1994.

[51] Clayton Petsche, Lucien Szpiro, and Michael Tepper. Isotriviality is equivalent to potential good reduction for endomorphisms of $\mathbb{P}^{N}$ over function fields. $J$. Algebra, 322(9):3345-3365, 2009.

[52] Alfredo Poirier. Critical portraits for postcritically finite polynomials. Fund. Math., 203(2):107-163, 2009.

[53] Bjorn Poonen. The classification of rational preperiodic points of quadratic polynomials over $\mathbb{Q}$ : a refined conjecture. Math. Z., 228(1):11-29, 1998.

[54] Mary Rees. A partial description of parameter space of rational maps of degree two. I. Acta Math., 168(1-2):11-87, 1992.

[55] Mary Rees. A partial description of the parameter space of rational maps of degree two. II. Proc. London Math. Soc. (3), 70(3):644-690, 1995.

[56] Joseph Silverman. Good reduction and Shafarevich-type theorems for dynamical systems with portrait level structures. Pacific J. Math., 295(1):145-190, 2018

[57] Joseph H. Silverman. The space of rational maps on $\mathbb{P}^{1}$. Duke Math. J., 94(1):41-77, 1998.

[58] Joseph H. Silverman. The Arithmetic of Dynamical Systems, volume 241 of Graduate Texts in Mathematics. Springer, New York, 2007.

[59] Joseph H. Silverman. Moduli Spaces and Arithmetic Dynamics, volume 30 of CRM Monograph Series. American Mathematical Society, Providence, RI, 2012.

[60] Tetsuo Ueda. Complex dynamics on projective spaces - index formula for fixed points. In Dynamical systems and chaos, Vol. 1 (Hachioji, 1994), pages 252259. World Sci. Publ., River Edge, NJ, 1995.

[61] Lloyd West. The moduli space of cubic rational maps, 2014.arXiv:1408.3247.

[62] Lloyd William West. The Moduli Space of Rational Maps. ProQuest LLC, Ann Arbor, MI, 2015. Thesis (Ph.D.)-City University of New York.

E-mail address: jdoyle@latech.edu 
Mathematics \& Statistics Department, Louisiana Tech University, Ruston, LA 71272 USA

E-mail address: jhs@math.brown.edu

Mathematics Department, Box 1917 Brown University, Providence, RI 02912 USA. ORCID: HTTPS://ORCID.ORG/0000-0003-3887-3248 